\title{
New Coherence Function with Measurements of One Sampling Period
}

\author{
J.M. Liu ${ }^{\mathrm{a}}$, Y.F. Xu ${ }^{\mathrm{b}}$, W.D. Zhu ${ }^{\mathrm{b}, *}$ \\ ${ }^{a}$ China Orient Institute of Noise and Vibration, Beijing 100085, China \\ ${ }^{b}$ Department of Mechanical Engineering, University of Maryland, Baltimore County, \\ 1000 Hilltop Circle, Baltimore, MD 21250, USA
}

\begin{abstract}
The conventional coherence function has been widely used to evaluate qualities of frequency response functions (FRFs). It requires response and excitation series of at least two sampling periods to yield meaningful function values. In addition, errors in a FRF and its associated IRF that are caused by system errors, such as response advancement error between measured response and excitation series in a data acquisition system, can never be reflected in the conventional coherence function. A new coherence function is introduced to efficiently and physically evaluate qualities of FRFs, which can yield meaningful and stable values even when response and excitation series of only one sampling period are available, and it can reflect response advancement error between measured response and excitation series.

Keywords: experimental modal analysis, coherence function, frequency response function, discrete Fourier transform, response advancement error, periodic extension
\end{abstract}

\footnotetext{
*Corresponding author. Tel: +1 410455 3394; fax: +1 4104551052.

Email addresses: liujm@coinv.com (J.M. Liu), yxu2@umbc.edu (Y.F. Xu), wzhu@umbc.edu (W.D. Zhu)
}

Preprint submitted to Mechanical Systems and Signal Processing

June 12, 2016 


\section{Introduction}

Discrete frequency response functions (FRFs) of a dynamic system are usually obtained using response and excitation series that are experimentally measured, which can be used to determine response spectra of the system under certain excitation spectra and to extract modal parameters of the system $[1,2,3,4]$. Discrete impulse response functions (IRFs) can be calculated using a least-squares (LS) method and an iterative method that directly uses response and excitation series based on the discrete Duhamel's integral [5], and associated discrete FRFs can be calculated by applying the discrete Fourier transform (DFT) to the IRFs [2]. A more efficient way of calculating discrete FRFs, compared with the aforementioned methods, is to apply the DFT to both response and excitation series and calculate the FRFs using some formulations. In the DFT, a series to be transformed is virtually extended to have an infinite length and be periodic with a period equal to the length of the series [6]. Periodic extension in the DFT can lead to erroneous FRFs when extended response and excitation do not conform to the physics of the system. However, when excitation is in the form of impulses or burst signals and the duration of one sampling period is so long that associated response can decay to zero at the end of one sampling period, resulting FRFs can be accurately measured with the application of the DFT and error in FRFs caused by periodic extension vanish.

The conventional coherence function has been proposed to evaluate qualities of discrete FRFs at each measured frequency [1,2], where DFTs of response and excitation series are used, and it has been widely used in ex- 
perimental modal analysis $[7,8,9,10,11,12,13,14]$. A coherence function that has the same form of the conventional coherence function has been proposed to identify cutting tool wear and chatter, where bending vibration measured by two crossed accelerometers are used, and values of the coherence function reaching one at chatter and first natural frequencies indicate an onset of chatter and a severe wear stage of a cutting tool, respectively [15]. Response and excitation series of at least two sampling periods are needed to yield meaningful coherence function values, and stable function values often require response and excitation series of more sampling periods. A possible error in a data acquisition system (DAS) is response advancement error between measured response and excitation series. Response and excitation series measured by the DAS violate causality of a mechanical system. Response advancement error does not change the amplitude but the phase of a FRF measured by the DAS, compared with that of a FRF measured by a DAS without the error. However, response advancement error can never be reflected in the conventional coherence function due to its definition.

In this work, a new coherence function in Ref. [5], which is similar to those in Refs. [16, 17], is introduced to efficiently and physically evaluate qualities of FRFs: meaningful and stable function values can be obtained when response and excitation series of only one sampling period are available, and errors in the FRFs and associated IRFs caused by system errors, such as response advancement error between response and excitation series in a DAS, can be reflected and quantified in the frequency domain, which constitutes novelty of this work. A fitting index in Ref. [5] is used based on the new coherence function to evaluate overall qualities of the FRFs and IRFs. 


\section{FRFs and Coherence Functions}

Suppose an under-damped single-input-single-output system is under general excitation $f(t)$ with zero initial conditions. The excitation $f(t)$ to and response $y(t)$ of the system are discretely measured by a DAS with a sampling interval $\triangle t$, and an excitation series $f_{i}=f(i \triangle t)$, where $i=1,2, \ldots, n$, and a response series $y_{j}=y(j \triangle t)$, where $j=1,2, \ldots, 2 n-1$, are available; $n$ values of the IRF $h(t)$, i.e., $h_{i}=h[(i-1) \Delta t]$, where $i=1,2, \ldots, n$, can be calculated from the discrete Duhamel's integral:

$$
[f t]_{(2 n-1) \times n}[h t]_{n \times 1}=\left(\frac{1}{\triangle t}\right)[y t]_{(2 n-1) \times 1}
$$

where

$$
[f t]=\left[\begin{array}{cccc}
f_{1} & 0 & \cdots & 0 \\
f_{2} & f_{1} & \cdots & 0 \\
\vdots & \vdots & \ddots & \vdots \\
f_{n} & f_{n-1} & \ldots & f_{1} \\
0 & f_{n} & \ldots & f_{2} \\
\vdots & \vdots & \ddots & \vdots \\
0 & 0 & \cdots & f_{n}
\end{array}\right],[h t]=\left[\begin{array}{c}
h_{1} \\
h_{2} \\
\vdots \\
h_{n}
\end{array}\right],[y t]=\left[\begin{array}{c}
y_{1} \\
y_{2} \\
\vdots \\
y_{2 n-1}
\end{array}\right]
$$

The LS method can be used to obtain $h_{i}$ by solving Eq. (1) using MoorePenrose pseudoinverse of $[f t]$, denoted by $[f t]^{+}$:

$$
[h t]_{n \times 1}=[f t]_{n \times(2 n-1)}^{+}\left(\frac{1}{\triangle t}\right)[y t]_{(2 n-1) \times 1}
$$

where $[f t]^{+}=\left([f t]^{\mathrm{T}}[f t]\right)^{-1}[f t]^{\mathrm{T}}$, in which the superscript $\mathrm{T}$ denotes transpose of a matrix [18]. Since matrix inversion is involved in calculating MoorePenrose pseudoinverse of $[f t]$, the LS solution is usually impractical due to its computational intensity. 
In practice, excitation and response series usually have the same length, and the matrix inversion can be avoided in calculating an IRF, based on the convolution theorem:

$$
h(t)=\mathscr{F}^{-1}[H(s)]
$$

where

$$
H(s)=\frac{Y(s)}{F(s)}
$$

defines the FRF associated with $h(t)$, in which $H(s)=\mathscr{F}[h(t)], Y(s)=\mathscr{F}[y(t)]$, and $F(s)=\mathscr{F}[f(t)]$, with $s$ and $\mathscr{F}(\cdot)$ denoting the excitation frequency in $\mathrm{Hz}$ and Fourier transform operation, respectively. Note that $f_{i}$ and $y_{i}$ have a finite length and the DFT is applied to $f_{i}$ and $y_{i}$ to obtain discrete $F(s)$ and $Y(s)$, respectively. The discrete $\mathrm{IRF} h_{i}$ can be obtained as the inverse DFT of discrete $F(s)$ based on Eq. (4). However, the FRF and associated IRF can be physically erroneous. The error results from periodic extension of $f_{i}$ in the DFT: $f_{i}$ is virtually extended to have an infinite length and becomes periodic with a period equal to its original length, i.e., $f_{i+l m}=f_{i}$, where $l$ is an integer. The resulting IRF $[h t]$ associated with $H(s)$ using measurement of one sampling period, which is obtained from Eq. (4), can be found from

$$
\left[f t^{p}\right]_{n \times n}[h t]_{n \times 1}=\left(\frac{1}{\triangle t}\right)[y t]_{n \times 1}
$$

where

$$
\left[f t^{p}\right]=\left[\begin{array}{ccccc}
f_{1} & f_{n} & f_{n-1} & \cdots & f_{2} \\
f_{2} & f_{1} & f_{n} & \cdots & f_{3} \\
\vdots & \vdots & \vdots & \ddots & \vdots \\
f_{n-1} & f_{n-2} & f_{n-3} & \cdots & f_{n} \\
f_{n} & f_{n-1} & f_{n-2} & \cdots & f_{1}
\end{array}\right]
$$


is the force matrix that corresponds to periodic excitation with a period equal to the sampling period; the response vector $[y t]$ corresponds to the transient rather than steady-state response of the system. As a result, $[h t]$ in Eq. (6) can be erroneous, since $\left[f^{p}\right]$ can violate the physics of the system due to the periodic extension. The error and measurement noise in a FRF can be reduced by use of measurements of multiple sampling periods [2], and the associated FRF can then be calculated using two formulations:

$$
H_{1}(s)=\frac{\bar{G}_{F Y}(s)}{\bar{G}_{F F}(s)}
$$

and

$$
H_{2}(s)=\frac{\bar{G}_{Y Y}(s)}{\bar{G}_{Y F}(s)}
$$

where

$$
\begin{gathered}
\bar{G}_{F Y}(s)=\bar{G}_{Y F}^{*}(s)=\frac{\sum_{k=1}^{N_{s}} F_{k}^{*}(s) Y_{k}(s)}{N_{s}} \\
\bar{G}_{F F}(s)=\frac{\sum_{k=1}^{N_{s}} F_{k}^{*}(s) F_{k}(s)}{N_{s}}
\end{gathered}
$$

and

$$
\bar{G}_{Y Y}(s)=\frac{\sum_{k=1}^{N_{s}} Y_{k}^{*}(s) Y_{k}(s)}{N_{s}}
$$

in which $N_{s}$ is the total number of sampling periods, $Y_{k}(s)$ and $F_{k}(s)$ are DFTs of $y_{i}$ and $f_{i}$ in the $k$-th sampling period, respectively, and the overbar and the superscript $*$ denote averaging and complex conjugation, respectively. When $f(t)$ is in the form of impulses or burst signals and the duration of one sampling period is so long that all entries after the $n$-th entry of $[y t]$ in Eq. (1) are almost zero, the IRF from Eq. (6) and associated FRF in 
one sampling period can be accurately estimated from Eq. (8) or (9). The reason is that both response and excitation can be completely measured in the sampling period and Eq. (6) conforms to the physics of the system, i.e., $y_{i}$ in $[y t]$ is only caused by $f_{i}, f_{i-1}, \ldots, f_{1}$.

The conventional coherence function to evaluate qualities of a FRF at each measured frequency is defined as the ratio between $H_{1}(s)$ and $H_{2}(s)$ and can be expressed by [2]

$$
\gamma^{2}(s)=\frac{H_{1}(s)}{H_{2}(s)}=\frac{\bar{G}_{F Y}(s) \bar{G}_{F Y}^{*}(s)}{\bar{G}_{F F}(s) \bar{G}_{Y Y}(s)}
$$

When response and excitation series of only one sampling period are available, both $H_{1}(s)$ and $H_{2}(s)$ are equal to $H(s)$ in Eq. (5), and the conventional coherence function yields meaningless values of one at all measured frequencies, regardless of qualities of the FRF. Response and excitation series of at least two sampling periods are needed to yield meaningful function values, and those of more sampling periods are often needed to yield stable function values.

One type of system errors that can exist in a DAS is response delay error, where measured response of a mechanical system occurs after excitation with a delay longer than a physical delay that is determined by the system and locations of response and excitation. However, one cannot differentiate between a delay resulting from the error and the physical one based on the measured response and excitation. Another type of system errors that can exist in a DAS is response advancement error, where measured response occurs before excitation, which violates causality of a mechanical system. In the measurement of a DAS with response advancement error, a response series $y_{i}$ occurs before an excitation series $f_{i}$. When $y_{i}$ occurs $m \Delta t$ earlier than 
$f_{i}$, i.e., $f_{i}$ occurs $m \Delta t$ later than $y_{i}$, in the DAS and a FRF between $y_{i}$ and $f_{i}$ can be calculated from Eq. (5), its associated IRF based on Eq. (6) can be found from

$$
\left[f t^{p}\right]_{n \times n}\left[h t^{d}\right]_{n \times 1}=\left(\frac{1}{\triangle t}\right)\left[y t^{d}\right]_{n \times 1}
$$

where $\left[h t^{d}\right]=\left[\begin{array}{llllllll}h_{m+1} & h_{m+2} & \ldots & h_{n-m} & h_{1} & h_{2} & \ldots & h_{m}\end{array}\right]^{\mathrm{T}}$ and $\left[y t^{d}\right]=$ $\left[\begin{array}{llllllll}y_{m+1} & y_{m+2} & \ldots & y_{n-m} & y_{1} & y_{2} & \ldots & y_{m}\end{array}\right]^{\mathrm{T}}$. While $\left[f t^{p}\right]$ in Eq. (14) is identical to that in Eq. (6), it can be seen that $\left[y t^{d}\right]$ has the same length as $[y t]$ in Eq. (6) and its first $n-m$ and last $m$ entries are the last $n-m$ and first $m$ entries of $[y t]$ due to the periodic extension of the DFT. Compared with the DFT of $y_{i}$, phases of the DFT of $\left[y t^{d}\right]$ are changed at all frequencies, while its amplitudes remain unchanged. When a FRF is calculated from Eqs. (8) or (9) using $y_{i}$ and $f_{i}$ measured by the DAS, $\bar{G}_{F F}(s)$ and $\bar{G}_{Y Y}(s)$ remain unchanged, but $\bar{G}_{F Y}(s)$ and $\bar{G}_{Y F}(s)$ change; the FRF and its associated IRF are erroneous. The error does not affect amplitudes but phases of the FRF at all frequencies. Effects of the error can be directly identified in $\left[h t^{d}\right]$, since its first $n-m$ and last $m$ entries are the last $n-m$ and first $m$ entries of $[h t]$ in Eq. (6). The effects exhibit themselves as oscillation at the end of $\left[h t^{d}\right]$. Use of the conventional coherence function in Eq. (13) can never assist recognition of the error. The reason is that the conventional coherence function is defined using $\bar{G}_{Y Y}(s), \bar{G}_{F F}(s)$ and squared amplitudes of $\bar{G}_{F Y}(s)$, which do not change when the error occurs. Hence values of the conventional coherence function do not change at all measured frequencies when $m \geq 1$, compared with those when $m=0$, i.e., the DAS does not have the error. 
A new coherence function is introduced to efficiently and physically evaluate qualities of a FRF and its IRF in Ref. [5]

$$
\gamma^{2}(s)=\frac{\bar{G}_{Y Y}}{\bar{G}_{Y Y}+\bar{G}_{E E}}
$$

where

$$
\bar{G}_{E E}(s)=\frac{\sum_{k=1}^{N_{s}} E_{k}^{*}(s) E_{k}(s)}{N_{s}}
$$

in which $E_{k}(s)$ is the DFT of an error series $e_{i}$ in the $k$-th sampling period. The error series $e_{i}$ can be obtained by

$$
e_{i}=y_{i}-\hat{y}_{i}
$$

where $\hat{y}_{i}$ is a predicted response series of a sampling period; the predicted response series is obtained as the first $n$ terms of the discrete Duhamel's integral in Eq. (1) between $f_{i}$ of the sampling period and $h_{i}$ associated with the FRF. Similar to the conventional coherence function, a FRF can be considered to be accurately measured at a frequency when the new coherence function value is one at the frequency; the lower the new coherence function value the less accurately measured the FRF at the frequency. The new coherence function is superior to the conventional one in two aspects. One is that meaningful coherence function values can be obtained with response and excitation series of one sampling period, such as a single impact and its associated response in one sampling period, which can greatly shorten the test time. The reason is that an error series is not necessarily a zero series even with only one sampling period due to measurement noise and 
error, and associated $\bar{G}_{E E}$ is not zero at some frequencies. The other is that the new coherence function can be used to recognize response advancement error due to the definition of the error series. The reason is that a predicted response series based on Eq. (1) conforms to the causality and the measured one can violate it; $\bar{G}_{E E}$ can be used to physically quantify the violation. Morever, one can reject measurements of one sampling period during an impact test based on the new coherence function, which facilitates efficient measurement of accurate FRFs. Note that both the conventional and new coherence functions cannot reflect response delay error. A similar form of coherence function to evaluate qualities of power spectra has been proposed in Ref. [16], where response series of at least two sampling periods are needed to yield meaningful values. Note that power spectra and FRFs are used in operational and experimental modal analysis, respectively.

The overall quality of a FRF can be evaluated using a fitting index based on the coherence function in Eq. (15), which is given by [5]

$$
f i t=\sqrt{\frac{\Xi \bar{G}_{Y Y}(s)}{\Xi \bar{G}_{E E}(s)+\bar{G}_{Y Y}(s)}}
$$

where $\Xi(\cdot)$ denotes summation over all frequencies. When $f i t=1$, values of the new coherence function are one at all frequencies; the lower the index the worse the overall quality of a FRF.

\section{Numerical and Experimental Investigation}

\subsection{Numerical investigation}

A numerical simulation is conducted on a two-degree-of-freedom massspring-damper system, as shown in Fig. 1 , with masses $m_{1}=1 \mathrm{~kg}$ and $m_{2}=2$ 
$\mathrm{kg}$; spring constants $k_{1}=6240 \pi \mathrm{N} / \mathrm{m}, k_{2}=4160 \pi \mathrm{N} / \mathrm{m}$, and $k_{3}=3120 \pi$ $\mathrm{N} / \mathrm{m}$; and viscous damping coefficients $c_{1}=2 \mathrm{~N} /(\mathrm{m} / \mathrm{s}), c_{2}=1 \mathrm{~N} /(\mathrm{m} / \mathrm{s})$, and $c_{3}=2 \mathrm{~N} /(\mathrm{m} / \mathrm{s})$. An external zero-mean burst random excitation $f(t)$ acts on the mass $m_{1}$; the duration of $f(t)$ in one sampling period is four seconds and it has a standard deviation of $14.5 \mathrm{~N}$. Responses of the two masses in the form of displacements, denoted by $y_{1}(t)$ and $y_{2}(t)$, are obtained with a sampling frequency of $256 \mathrm{~Hz}$ by solving the associated set of ordinary differential equations with zero initial conditions:

$$
\begin{aligned}
& m_{1} \ddot{y}_{1}(t)+\left(c_{1}+c_{2}\right) \dot{y}_{1}(t)-c_{2} \dot{y}_{2}(t)+\left(k_{1}+k_{2}\right) y_{1}(t)-k_{2} y_{2}(t)=f(t) \\
& m_{2} \ddot{y}_{2}(t)-c_{2} \dot{y}_{1}(t)+\left(c_{2}+c_{3}\right) \dot{y}_{2}(t)-k_{2} y_{1}(t)+\left(k_{2}+k_{3}\right) y_{2}(t)=0 \\
& y_{1}(0)=0, \dot{y}_{1}(0)=0, y_{2}(0)=0, \dot{y}_{2}(0)=0
\end{aligned}
$$

where an overdot denotes time differentiation.

Responses of the two masses are calculated in five sampling periods. The duration of one sampling period is $16 \mathrm{~s}$, and it is so long that a FRF of the system can be accurately estimated using Eq. (8) or (9). White noise is added to the responses with a signal-to-noise ratio of $80 \mathrm{db}$ to simulate severe measurement noise. The excitation $f(t)$ and $y_{1}(t)$ are shown in Figs. 2(a) and (b), respectively. A FRF between $y_{1}(t)$ and $f(t)$ from Eq. (8) of the first, first two and first five sampling periods are shown in Fig. 3(a) through (c), respectively. It can be seen that the FRFs are noisy due to the fact that the simulated measurement noise has a relatively high level. Conventional and new coherence functions associated with the FRFs using $y(t)$ and $f(t)$ of the first, first two and first five sampling periods are compared in Figs. 4(a) 
through (c), respectively. It can be seen in Fig. 4(a) that the new coherence function has meaningful values when $y_{1}(t)$ and $f(t)$ of only the first sampling period are used, while the conventional one has a meaningless value of one at all frequencies. Fitting indices fit associated with the FRFs using $y(t)$ and $f(t)$ of the first, first two and first five periods were $89.91 \%, 95.95 \%$ and $95.58 \%$, respectively. 


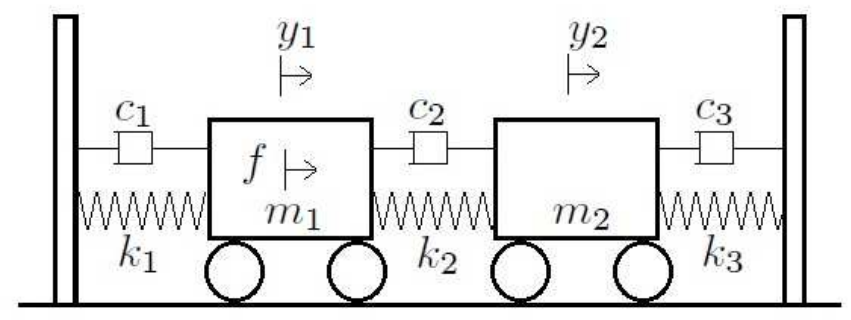

Figure 1: A two-degree-of-freedom mass-spring-damper system. 


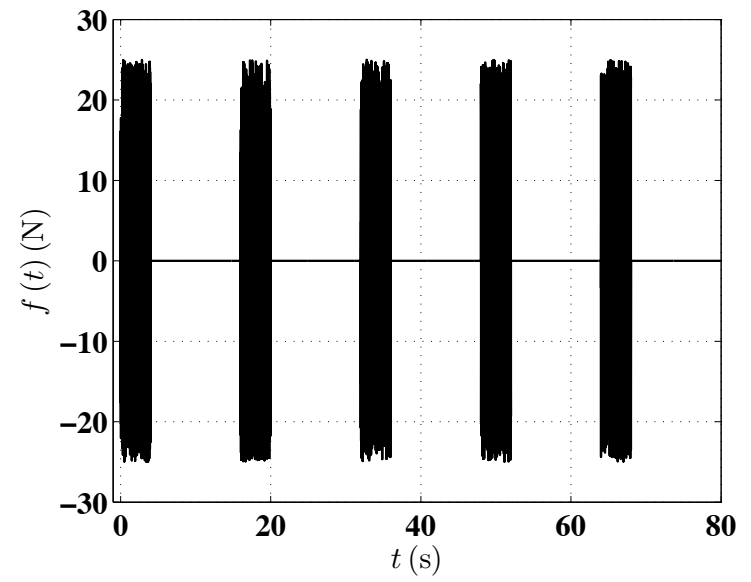

(a)

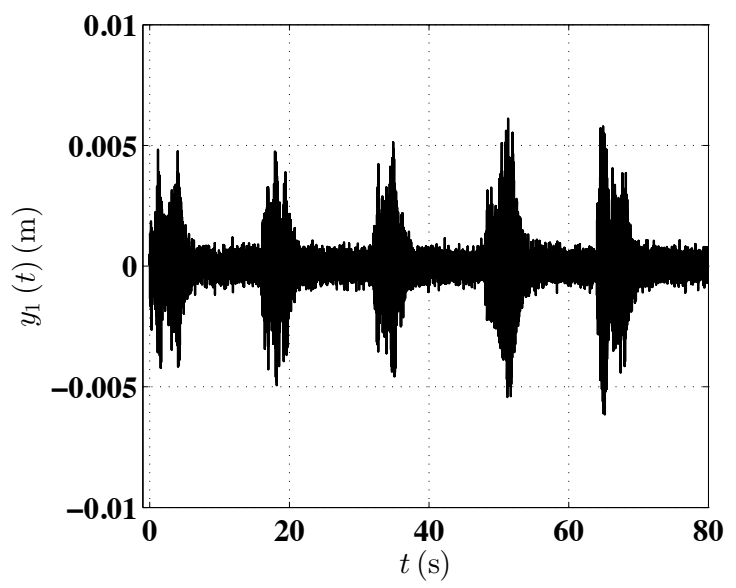

(b)

Figure 2: (a) Burst random excitation and (b) the response of $m_{1}$ in Fig. 1 of five sampling periods. 


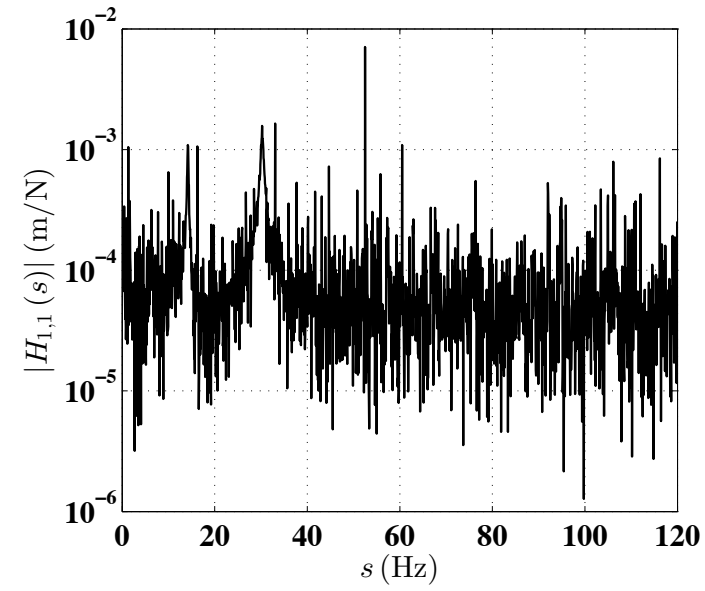

(a)

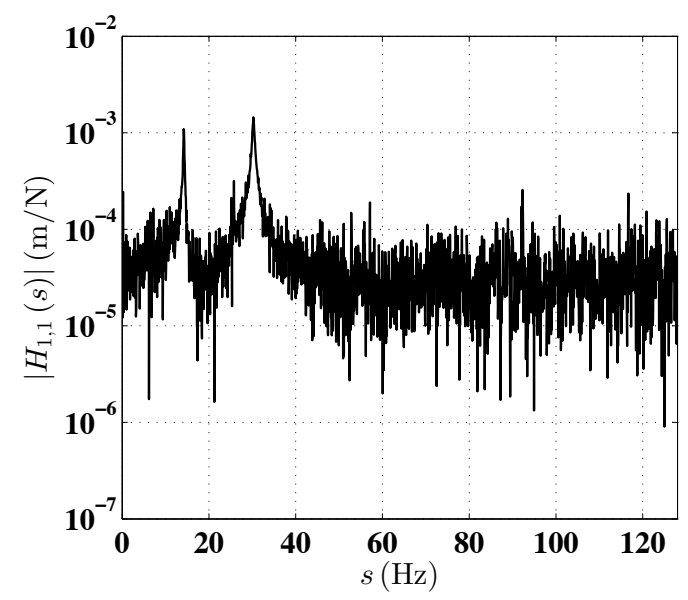

(c)

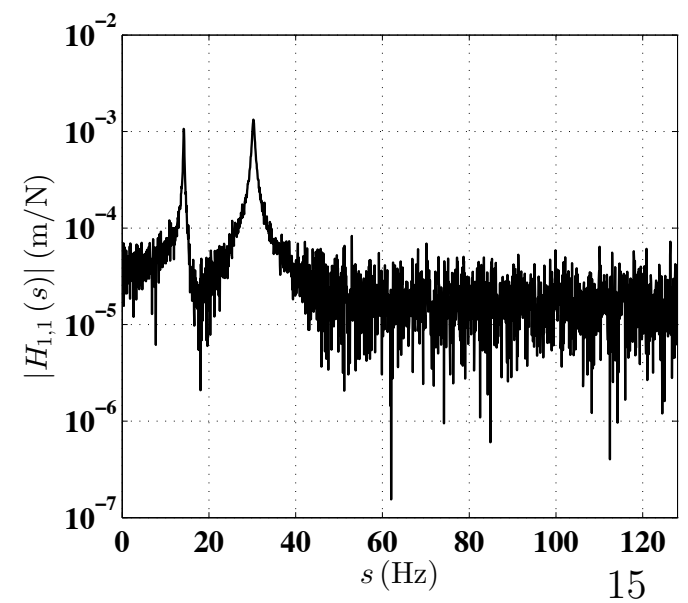

(e)

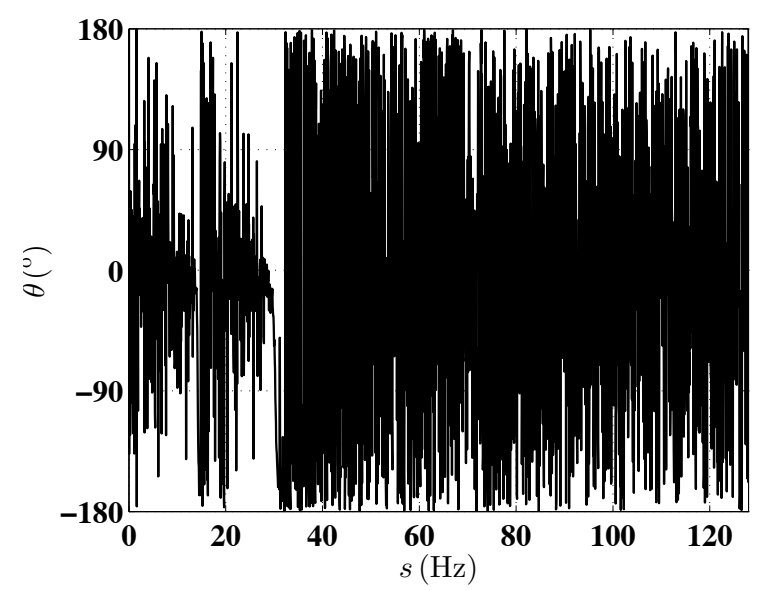

(b)

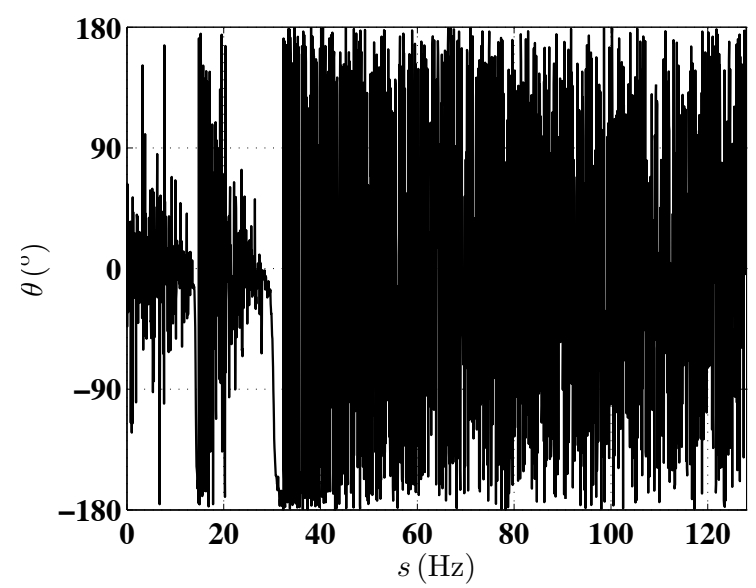

(d)

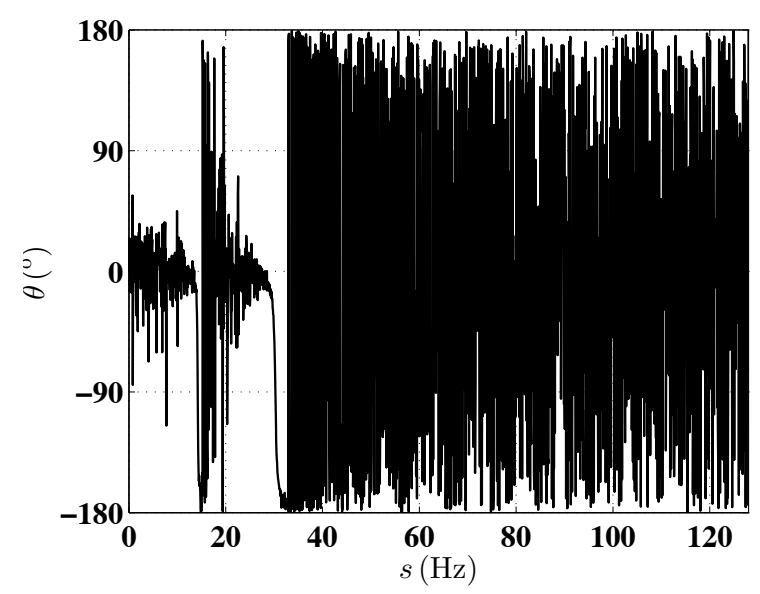

(f)

Figure 3: (a) Amplitude and (b) phase of the FRF using $y_{1}(t)$ and $f(t)$ of the first sampling period in Fig. 2, (c) the amplitude and (d) phase of the FRF using $y_{1}(t)$ and $f(t)$ of the first two sampling periods in Fig. 2, (e) the amplitude and (f) phase of the FRF using $y_{1}(t)$ and $f(t)$ of the first five sampling periods in Fig. 2. 
To visualize effects of response advancement error on a FRF and its associated IRF, occurrence of the excitation $f(t)$ is "postponed" by padding zero series of certain lengths to the start of $f(t)$ of each sampling period, while the length of a padded excitation series remains the same as that of the unpadded one. FRFs using $y_{1}(t)$ and $f(t)$ padded with zero series that have lengths of $0.00390625 \mathrm{~s}, 0.0078125 \mathrm{~s}$ and $0.01171875 \mathrm{~s}$ are calculated and shown in Fig. 5. It can be seen that amplitudes of the benchmark FRF and those using $y_{1}(t)$ and padded $f(t)$ are identical but phases of the FRFs are different due to the response advancement error between $y_{1}(t)$ and $f(t)$. IRFs associated with the aforementioned FRFs are compared in Fig. 6. The IRFs associated with the FRFs using $y_{1}(t)$ and padded $f(t)$ are shifted by durations equal to corresponding response advancement errors, compared with that associated with the benchmark FRF, as shown in Fig. 6. 


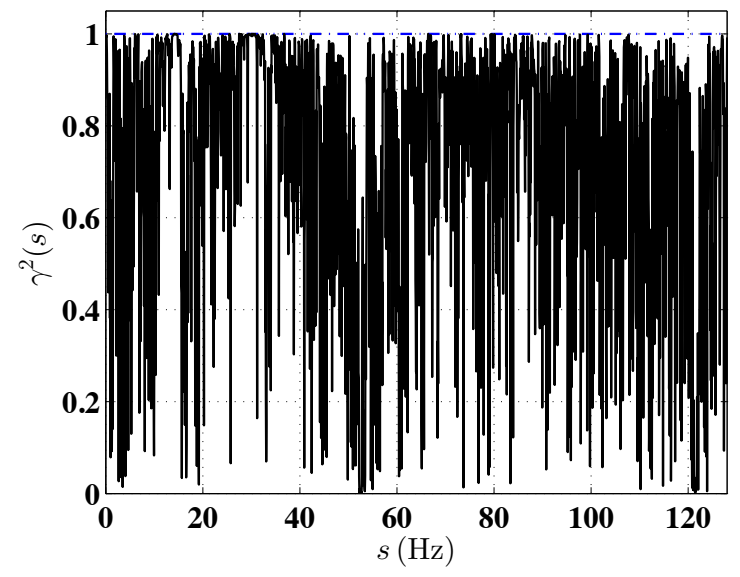

(a)

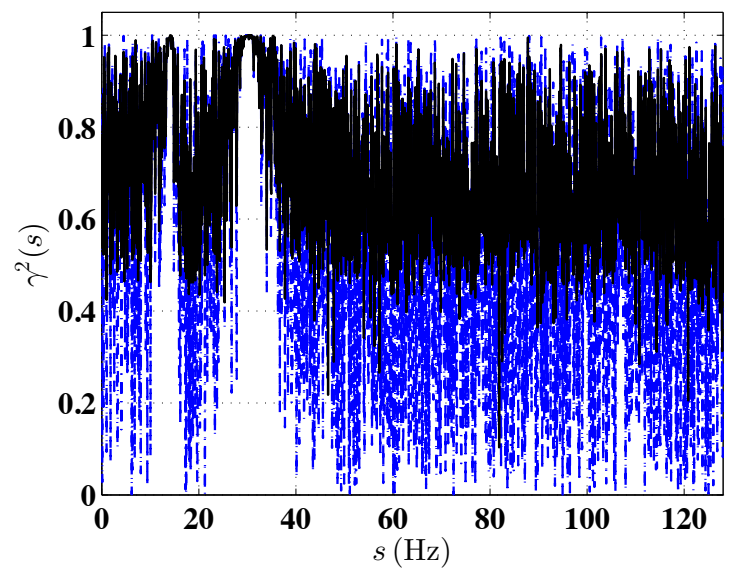

(b)

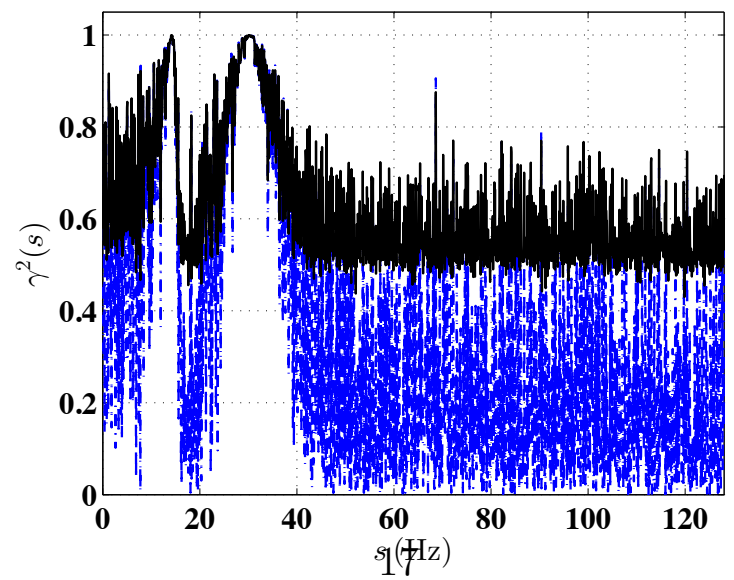

(c)

Figure 4: Comparisons of conventional (dashed line) and new (solid line) coherence functions using $y_{1}(t)$ and $f(t)$ of (a) the first sampling period, (b) the first two sampling periods and (c) the first five sampling periods. 
Conventional and new coherence functions associated with the FRFs using $y_{1}$ and $f(t)$ of five sampling periods are compared in Figs. 7 (a) through (c), where $f(t)$ are padded with zero series that have lengths of 0.00390625 s, $0.0078125 \mathrm{~s}$ and $0.01171875 \mathrm{~s}$, respectively. It can be seen that the conventional coherence functions do not change at all compared with that in Fig. 4(c), since a conventional coherence function reflects repeatability of response and excitation series, which cannot be changed by response advancement error. The new coherence function and fitting index can reflect the response advancement error as the longer the padded zero series the worse the coherence functions (Fig. 7), and $f i t$ associated with the FRFs using $y_{1}$ and $f(t)$ padded with zero series that have lengths of $0.00390625 \mathrm{~s}, 0.0078125 \mathrm{~s}$ and $0.01171875 \mathrm{~s}$ are $95.56 \%, 95.12 \%$ and $94.14 \%$, respectively.

\subsection{Experimental investigation}

\subsubsection{Test of a cantilever beam}

A FRF of an aluminum cantilever beam with damage, as shown in Fig. 8, was measured. The beam was of length $455.00 \mathrm{~mm}$, width $25.60 \mathrm{~mm}$ and thickness $6.50 \mathrm{~mm}$, and it had a region of machined thickness reduction on top and bottom surfaces along its length. The width of the region was the same as that of the beam, and its length and thickness were $55.70 \mathrm{~mm}$ and 4.30 $\mathrm{mm}$, respectively; thickness reduction on both the top and bottom surfaces of 
the region was $1.10 \mathrm{~mm}$. The right end of the beam was clamped by a bench vice to simulate a fixed boundary. The distance between the fixed end of the beam and the left end of the region was $251.70 \mathrm{~mm}$. A PCB 086D80 impact hammer was used to manually generate impacts $f(t)$ at the excitation point, and a PCB 352C66 accelerometer was used to measure accelerations $\ddot{z}(t)$ of the measurement point. The hammer and accelerometer were connected to a DAS, which was a LMS spectrum analyzer, and impacts and responses of five sampling periods were measured with a sampling frequency of $32768 \mathrm{~Hz}$. Durations of measured $f(t)$ and $\ddot{z}(t)$ of one sampling period were $32 \mathrm{~s}$. Time histories of $f(t)$ and $\ddot{z}(t)$ of the five sampling periods are shown in Figs. 9(a) and (b), respectively.

It can be observed in Fig. 9(b) that the duration of one sampling period was so long that the FRF could be accurately estimated using Eq. (8) or (9). FRFs from Eq. (8) using $\ddot{z}(t)$ and $f(t)$ of the first, first two and first five sampling periods are shown in Fig. 10, all of which were almost identical since there was not much excitation and measurement noise, and the FRF measurement was carefully conducted. Conventional and new coherence functions associated with the FRFs using $\ddot{z}(t)$ and $f(t)$ of the first, first two and first five sampling periods are compared in Figs. 11(a) through (c), respectively. It can be seen in Fig. 11(a) that the new coherence function had meaningful values when $\ddot{z}(t)$ and $f(t)$ of only the first sampling period were used, while the conventional one had a meaningless value of one at 
all frequencies. Comparing coherence functions in Figs. 11(a) through (c), one can observe that the new coherence function had stable values, since it changed less than the conventional one with the increase of the number of sampling periods. Fitting indices fit associated with the FRFs using $\ddot{z}(t)$ and $f(t)$ of the first, first two and first five periods were $99.90 \%, 99.82 \%$ and $99.70 \%$, respectively, indicating that the FRF measurement had a good overall quality.

Occurrence of impacts in $f(t)$ was "postponed" by padding zero series of certain lengths to the start of $f(t)$ of each sampling period, while the length of a padded excitation series remain the same as that of the unpadded one. FRFs using $\ddot{z}(t)$ and $f(t)$ padded with zero series that had lengths of $0.001 \mathrm{~s}, 0.005 \mathrm{~s}$ and $0.01 \mathrm{~s}$ were calculated and shown in Fig. 12. It can be seen that amplitudes of the benchmark FRF and those using $\ddot{z}(t)$ and padded $f(t)$ were identical but phases of the FRFs were different due to the response advancement error between $\ddot{z}(t)$ and $f(t)$. IRFs associated with the aforementioned FRFs are compared in Fig. 13. It can be observed in Fig. 13(b) that there were oscillation in the IRF associated with the benchmark FRF at the end of a sampling period, indicating that the current DAS could have response advancement error. The IRFs associated with the FRFs using $\ddot{z}(t)$ and padded $f(t)$ were shifted by durations equal to corresponding response advancement errors compared with that associated with the benchmark FRF, as shown in Fig. 13. 
Conventional and new coherence functions associated with the FRFs using $\ddot{z}(t)$ and $f(t)$ of five sampling periods are compared in Figs. 14 (a) through $(\mathrm{c})$, where $f(t)$ were padded with zero series that had lengths of $0.001 \mathrm{~s}, 0.005 \mathrm{~s}$ and $0.01 \mathrm{~s}$, respectively. It can be seen that the conventional coherence functions did not change at all compared with that in Fig. 11 (c) since a conventional coherence function reflected repeatability of response and excitation series, which could not be changed by the response advancement error. Note that peaks in the new coherence function occurred nearly periodically in Figs. 14 (b) and (c), which could be a coincidence, since this behavior could not be observed in the numerical example in Sec. 3.1 and the other experimental example in the following section. The new coherence function and fitting index could reflect the response advancement error as the longer the padded zero series the worse the coherence functions (Fig. 14), and $f i t$ associated with the FRFs using $\ddot{z}(t)$ and $f(t)$ padded with zero series that had lengths of $0.001 \mathrm{~s}, 0.005 \mathrm{~s}$ and $0.01 \mathrm{~s}$, as shown in Fig. 14, were $98.58 \%, 96.71 \%$ and $95.23 \%$, respectively.

\subsubsection{Test of an outdoor rail}

A FRF of an outdoor aluminum rail at the University of Maryland, Baltimore County was measured with excitation and response points shown in Fig. 15. A PCB 086D20 hammer was used to manually generate impacts 
$f(t)$ at the excitation point, and a PCB 352C66 accelerometer was used to measure accelerations $\ddot{z}(t)$ of the measurement point. The hammer and accelerometer were connected to the spectrum analyzer, and impacts and responses of five sampling periods were measured with a sampling frequency of $256 \mathrm{~Hz}$. Duration of measured $f(t)$ and $\ddot{z}(t)$ of one sampling period were $64 \mathrm{~s}$. Time histories of $f(t)$ and $\ddot{z}(t)$ of the five sampling periods are shown in Figs. 16(a) and (b), respectively.

While amplitudes of $\ddot{z}(t)$ of the five periods exponentially decayed, some of them did not approach zero at their ends due wind excitation, since the rail was tested on a windy day with a reported average wind speed of 19 mph. An exponential window that can be expressed as

$$
w(t)=\mathrm{e}^{-0.04 t}
$$

was applied to $\ddot{z}(t)$ of each period before calculating the FRF to avoid leakage in its DFT. Windowed $\ddot{z}(t)$ is shown in Fig. 16(c), and it can be seen that windowed $\ddot{z}(t)$ of each sampling period approached zero at its end. The duration of one sampling period was so long that the FRF could be accurately estimated from Eq. (8). FRFs using $\ddot{z}(t)$ and $f(t)$ of the first, first two and first five sampling periods are shown in Fig. 17, and conventional and new coherence functions associated with the FRFs using $\ddot{z}(t)$ and $f(t)$ of the first, first two and first five periods are shown in Figs. 18(a) through (c), respectively. Similar to the test of the cantilever beam, the new coherence 
function had meaningful values when $\ddot{z}(t)$ and $f(t)$ of only the first period were used, while the conventional one had meaningless values of one at all frequencies. Comparing the coherence functions in Fig. 18, one can observe that the new coherence function had stable values, since it changed less than the conventional one with an increase in the number of sampling periods, especially at low frequencies. Fitting indices $f i t$ associated with the FRFs using $\ddot{z}(t)$ and $f(t)$ of the first, first two and first five periods were $99.99 \%$, 99.66\% and 99.48\%, respectively, which indicates that the FRFs had good overall qualities. IRFs associated with the FRFs using $\ddot{z}(t)$ and $f(t)$ of the first, first two and first five periods between $30 \mathrm{~s}$ and $60 \mathrm{~s}$ are shown in Fig. 19, where an increase in the number of sampling periods improved the IRF qualities, since the larger the number of sampling periods, the lower the noise level of an IRF.

Occurrence of impacts in $f(t)$ was then "postponed" by padding zero series of lengths of one, two and three to the start of $f(t)$ of each sampling period, which corresponds to response advancement errors of $0.00390625 \mathrm{~s}$, $0.0078125 \mathrm{~s}$ and $0.01171875 \mathrm{~s}$, respecitvely. FRFs using $\ddot{z}(t)$ and padded $f(t)$ are calculated and shown in Fig. 20, and a benchmark FRF using $\ddot{z}(t)$ and unpadded $f(t)$ is shown in Fig. 20. Similar to the test of the cantilever beam, amplitudes of the benchmark FRF and those of the FRFs using $\ddot{z}(t)$ 
and padded $f(t)$ were identical, but their phases were different due to the response advancement errors. IRFs associated with the aforementioned FRFs are compared in Fig. 21. There were oscillations at the end of a sampling period, which confirms a possible response advancement error in the DAS. IRFs associated with the FRFs using $\ddot{z}(t)$ and padded $f(t)$ were shifted by durations equal to corresponding response advancement errors compared with that associated with the benchmark FRF, as shown in Fig. 21.

Conventional and new coherence functions associated with the FRFs using $\ddot{z}(t)$ and $f(t)$ padded with zero series that had the aforementioned lengths are shown in Fig. 22. Similar to the test of the cantilever beam, the conventional coherence functions did not change at all compared with that in Fig. 18(c), and the new coherence functions became worse with a longer padded zero series. Fitting indices $f i t$ associated with the FRFs using $\ddot{z}(t)$ and $f(t)$ padded with zeroe series that had lengths of $0.00390625 \mathrm{~s}, 0.00781250 \mathrm{~s}$ and 0.01171815 s were $98.79 \%, 98.88 \%$ and $98.75 \%$, respectively.

\section{Conclusion}

Errors in calculating FRFs of a mechanical system by applying the DFT to response and excitation series have been identified. A new coherence function and a fitting index based on the new coherence function are introduced 
to efficiently and physically evaluate qualities of a FRF. The new coherence function was applied to a calculated FRF of a two-degree-of-freedom massspring-damper system and measured FRFs of an aluminum cantilever beam and an outdoor rail. It has been shown that by use of the new coherence function meaningful and stable function values can be obtained using response and excitation series of only one sampling period and response advancement error in a DAS can be reflected, which can never be achieved by use of the conventional coherence function.

\section{Acknowledgment}

The authors are grateful for the financial support from the National Science Foundation under Grant Number CMMI-1335024. They would also like to thank Scott Smith for assistance on the experimental work.

\section{References}

[1] D. J. Ewins, Modal Testing: Theory, Practice and Application, 2nd Edition, Research Studies Press, Hertfordshire, UK, 2000.

[2] W. Heylen, S. Lammens, P. Sas, Modal Analysis Theory and Testing, Departement Werktuigkunde, Katholieke Universiteit, Leuven, Belgium, 1995.

[3] D. J. Inman, Vibration with Control, John Wiley \& Sons, Hoboken, NJ, 2006.

[4] R. Pintelon, J. Schoukens, System Identification: A Frequency Domain Approach, 2nd Edition, Wiley-IEEE Press, Hoboken, NJ, 2012. 
[5] J. M. Liu, W. D. Zhu, Q. H. Lu, G. X. Ren, An efficient iterative algorithm for accurately calculating impulse response functions in modal testing, Journal of Vibration and Acoustics 133 (6) (2011) 064505.

[6] W. L. Briggs, V. E. Henson, The DFT: An Owners' Manual for the Discrete Fourier Transform, Society for Industrial and Applied Mathematics, Philadelphia, PA, 1987.

[7] A. J. Felber, Development of a hybrid bridge evaluation system, Ph.D. thesis, University of British Columbia (1993).

[8] Q. Yang, H. Pang, Z. Wang, G. Lim, F. Yap, R. Lin, Vibration reliability characterization of pbga assemblies, Microelectronics Reliability 40 (7) (2000) 1097-1107.

[9] C. P. Ratcliffe, A frequency and curvature based experimental method for locating damage in structures, Journal of vibration and acoustics 122 (3) (2000) 324-329.

[10] M. I. Friswell, J. Penny, Crack modeling for structural health monitoring, Structural Health Monitoring 1 (2) (2002) 139-148.

[11] T. Nagayama, B. Spencer, G. Agha, K. Mechitov, Model-based data aggregation for structural monitoring employing smart sensors, in: Proceedings of the Third International Conference on Networked Sensing Systems (INSS 2006), May, 2006, pp. 203-210.

[12] R. Farshidi, D. Trieu, S. S. Park, T. Freiheit, Non-contact experimental modal analysis using air excitation and a microphone array, Measurement 43 (6) (2010) 755-765. 
[13] A. Tomaszewska, Influence of statistical errors on damage detection based on structural flexibility and mode shape curvature, Computers \& structures 88 (3) (2010) 154-164.

[14] A. G. A. Rahman, Z. C. Ong, Z. Ismail, Enhancement of coherence functions using time signals in modal analysis, Measurement 44 (10) (2011) 2112-2123.

[15] X. Q. Li, Y. S. Wong, A. Y. C. Nee, Tool wear and chatter detection using the coherence function of two crossed accelerations, International Journal of Machine Tools and Manufacture 37 (4) (1997) 425-435.

[16] Y. F. Xu, J. M. Liu, W. D. Zhu, Accurate and efficient calculation of discrete correlation functions and power spectra, Journal of Sound and Vibration 347 (2015) 246-265.

[17] M. J. Hinich, A statistical theory of signal coherence, Oceanic Engineering, IEEE Journal of 25 (2) (2000) 256-261.

[18] M. M. Ettouney, S. Alampalli, Infrastructure Health in Civil Engineering: Theory and Components, CRC Press, Boca Raton, FL, 2011. 


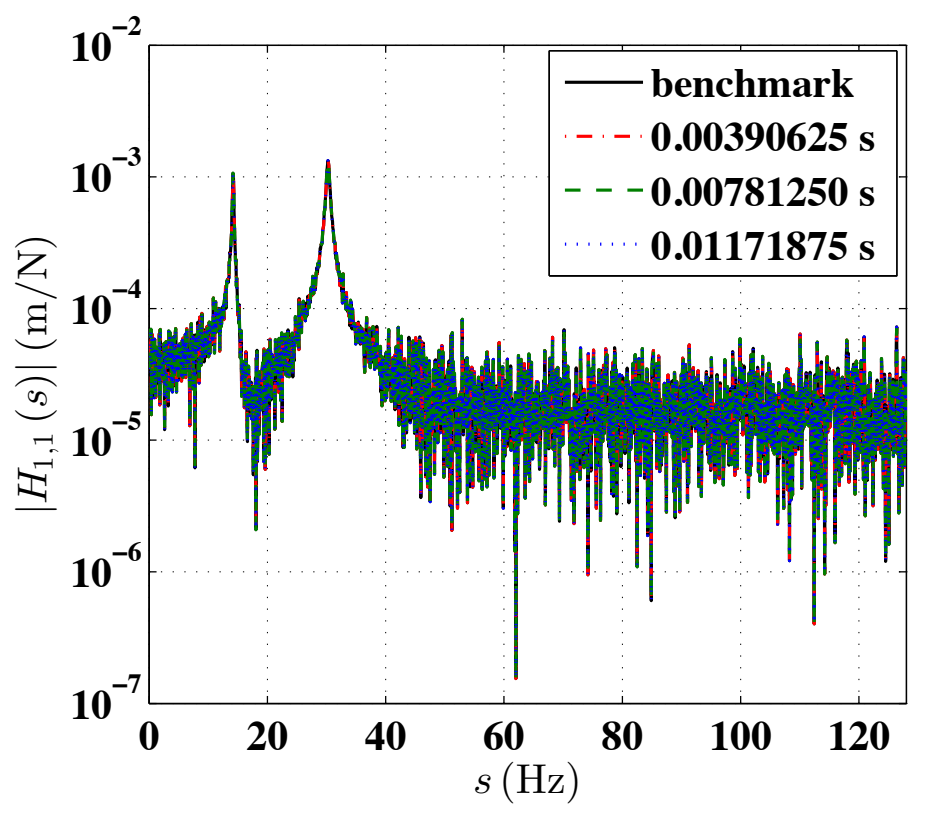

(a)

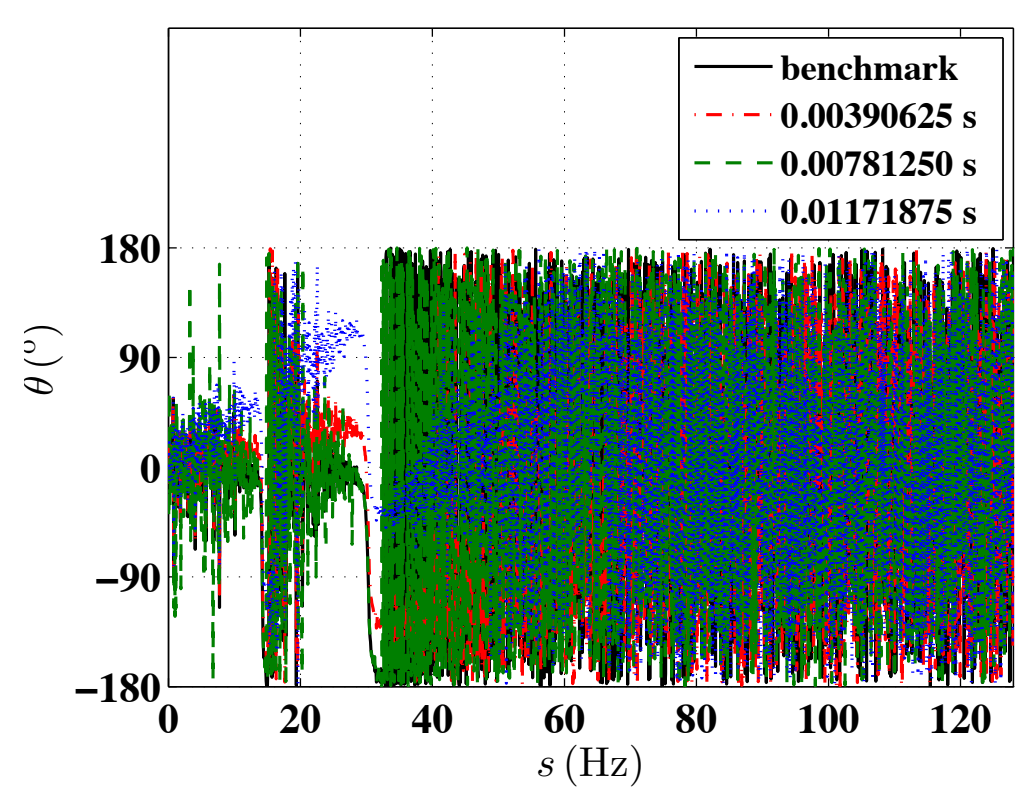

(b)

Figure 5: (a) Comparison of amplitudes of the benchmark FRF (benchmark) using $y_{1}(t)$ and $f(t)$ of the first five sampling periods in Fig. 2 and those using $y_{1}(t)$ and $f(t)$ padded with zero series that have lengths of $0.00390625 \mathrm{~s}, 0.0078125 \mathrm{~s}$ and $0.01171875 \mathrm{~s}$; and (b) comparison of their phases. 


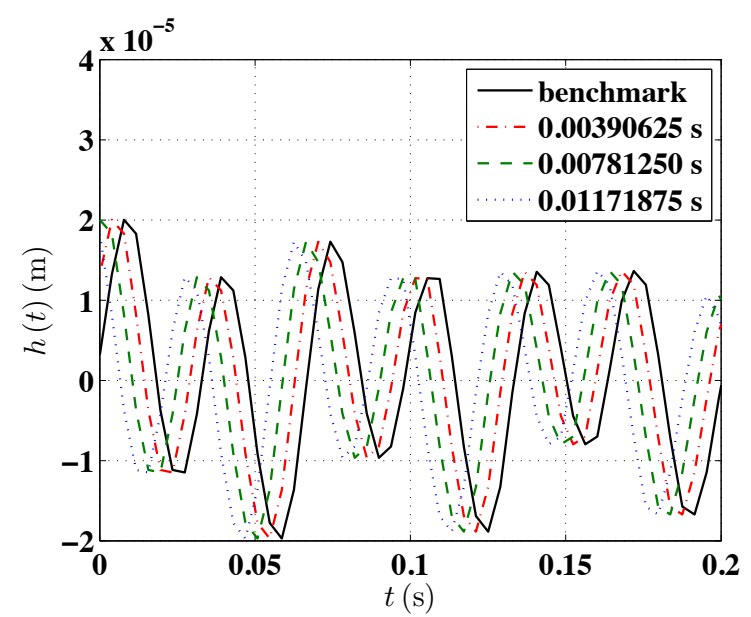

(a)

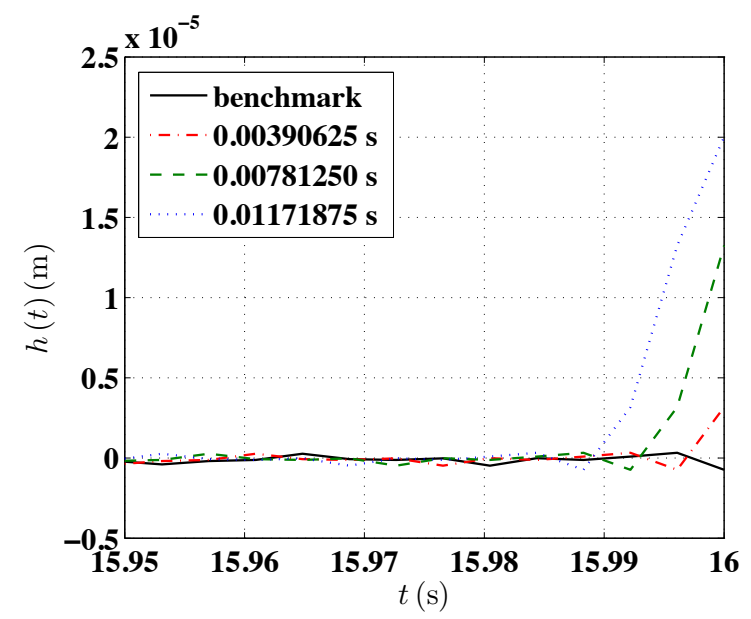

(b)

Figure 6: Comparison of IRFs associated with the benchmark FRF (benchmark) using $y_{1}(t)$ and $f(t)$ of the first five sampling periods in Fig. 2 and those using $y_{1}(t)$ and $f(t)$ padded with zero series that have lengths of $0.00390625 \mathrm{~s}, 0.0078125 \mathrm{~s}$ and $0.01171875 \mathrm{~s}$ in different time intervals: (a) between $t=0 \mathrm{~s}$ and $t=0.02 \mathrm{~s}$ and (b) between $t=15.95 \mathrm{~s}$ and $t=16 \mathrm{~s}$. 


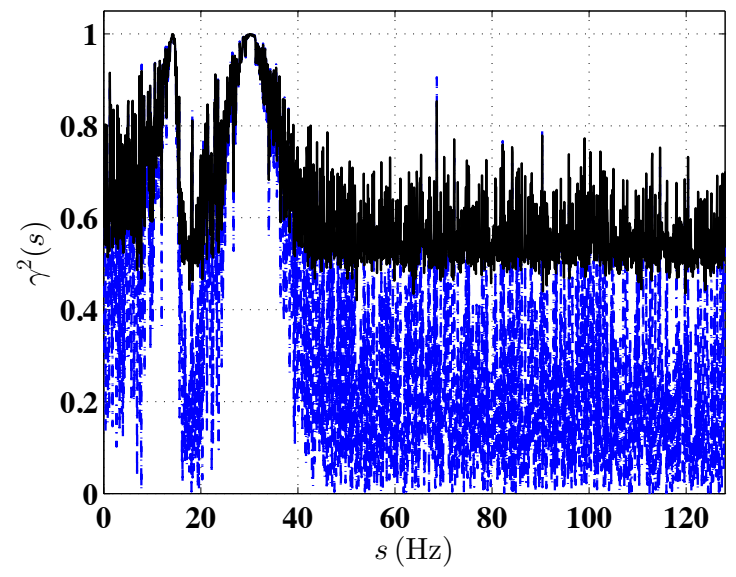

(a)

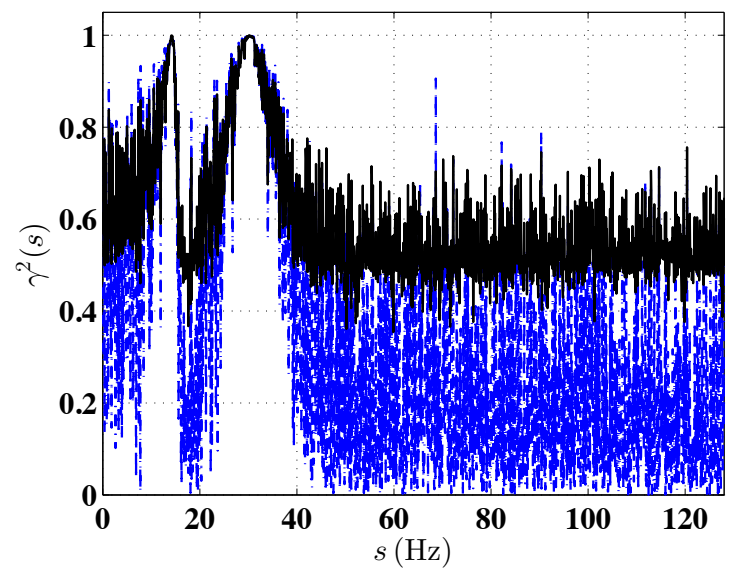

(b)

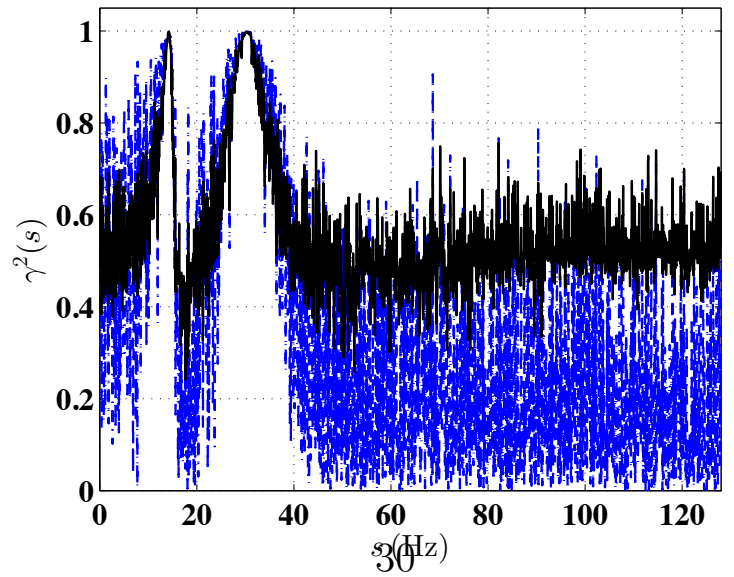

(c)

Figure 7: Comparisons of conventional and new coherence functions using $y_{1}(t)$ and $f(t)$ padded with zero series that have different lengths of: (a) $0.00390625 \mathrm{~s}$, (b) $0.0078125 \mathrm{~s}$ and (c) $0.01171875 \mathrm{~s}$. 


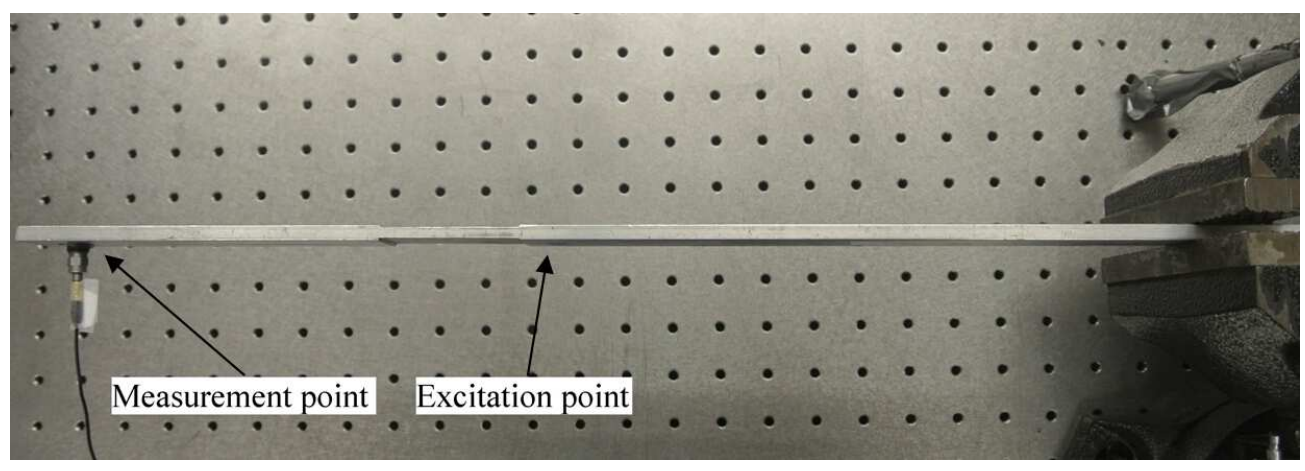

Figure 8: Test setup of an aluminum cantilever beam. 


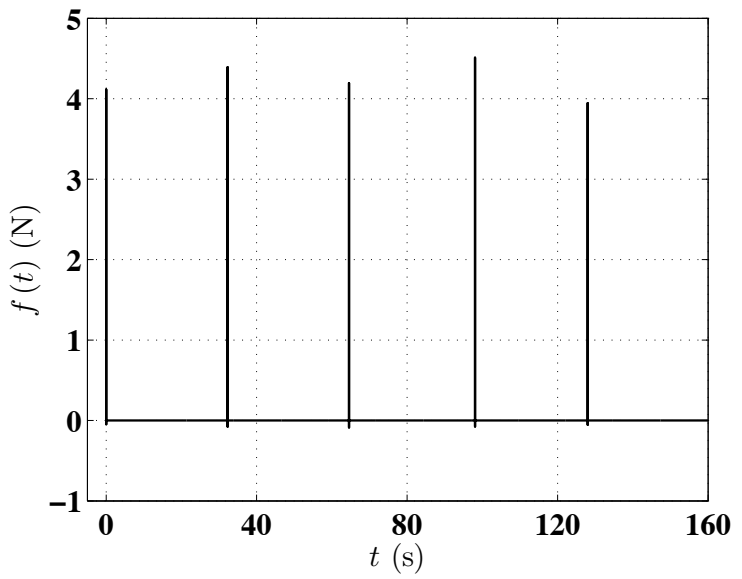

(a)

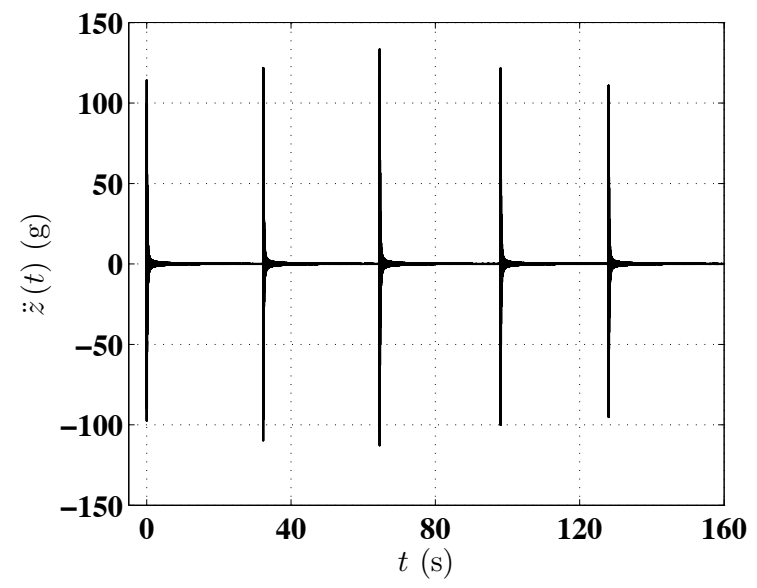

(b)

Figure 9: (a) Manually generated impacts at the excitation point on the beam in Fig. 8 and (b) responses of the measurement point on the beam in Fig. 8 of the five sampling periods. 


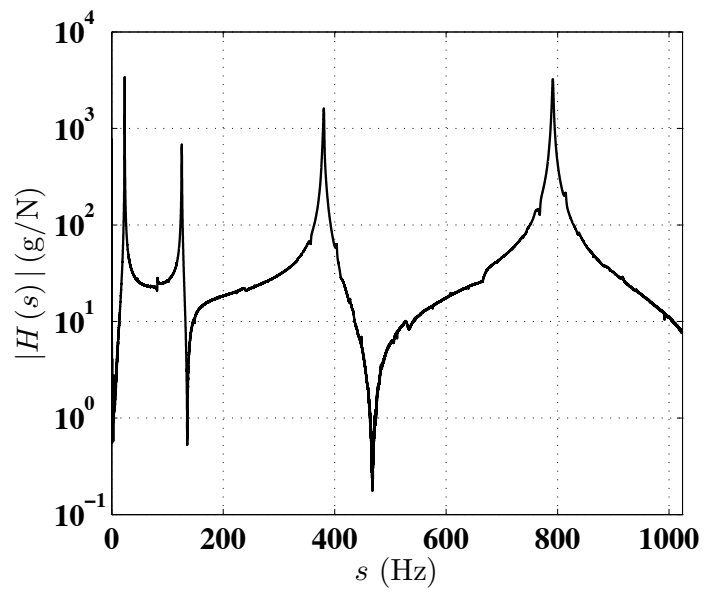

(a)

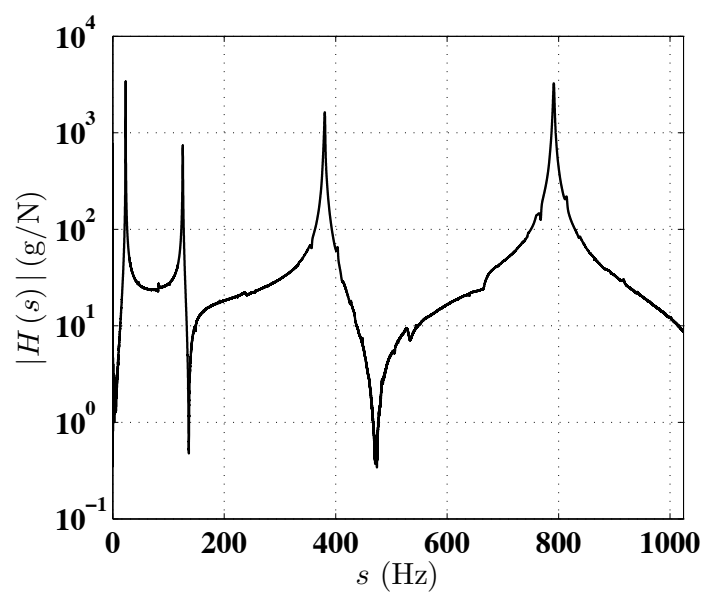

(c)

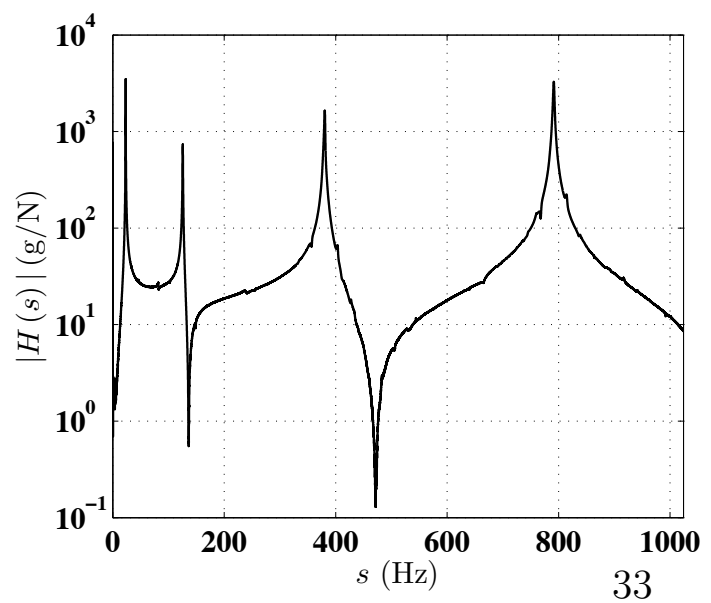

(e)

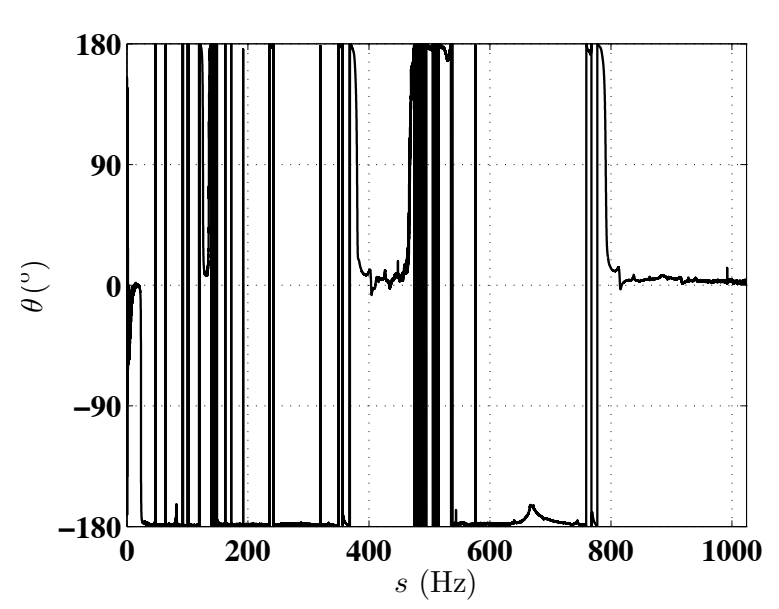

(b)

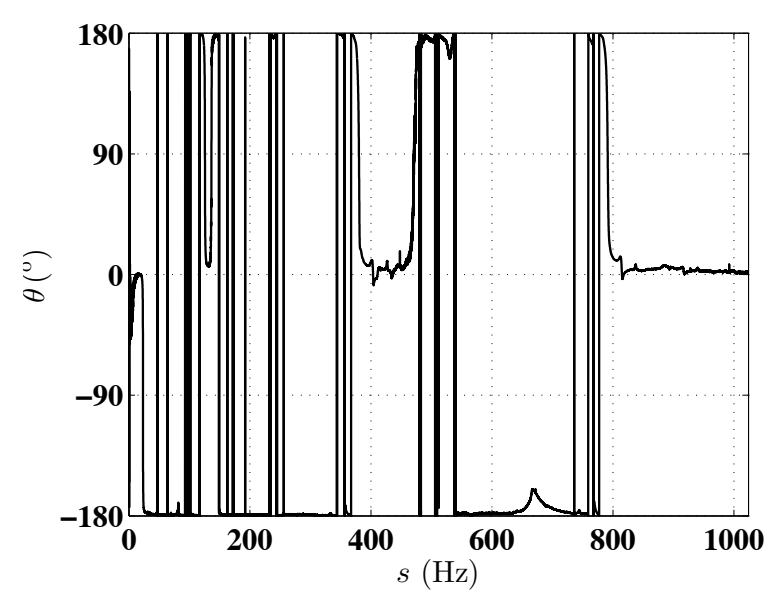

(d)

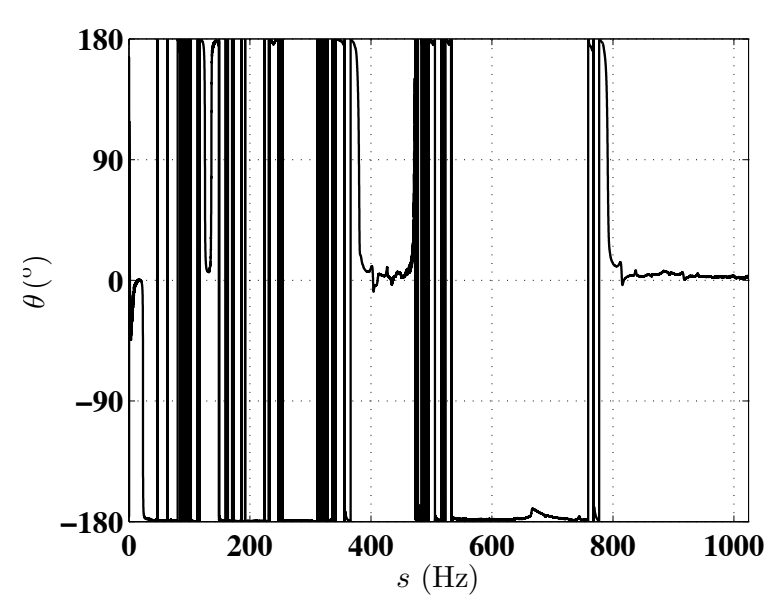

(f)

Figure 10: (a) Amplitude and (b) phase of the FRF using $\ddot{z}(t)$ and $f(t)$ of the first sampling period in Fig. 9, (c) the amplitude and (d) phase of the FRF using $\ddot{z}(t)$ and $f(t)$ of the first two sampling periods in Fig. 9, (e) the amplitude and (f) phase of the FRF using $\ddot{z}(t)$ and $f(t)$ of the first five sampling periods in Fig. 9. 


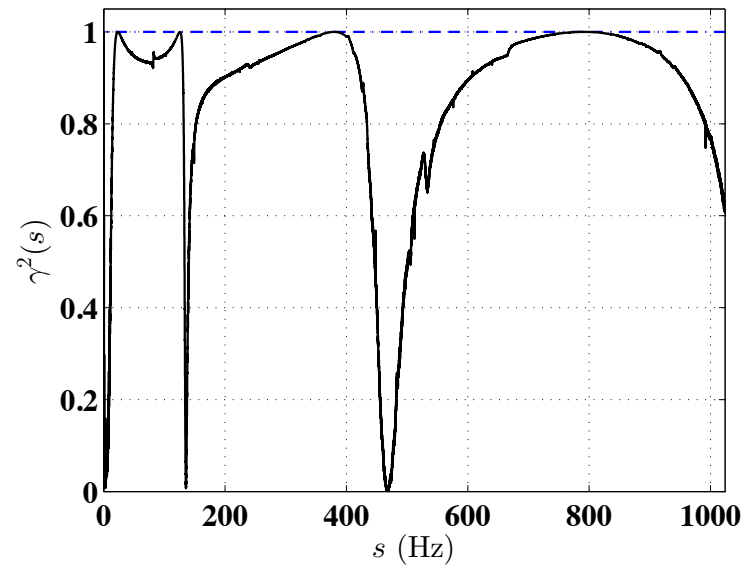

(a)

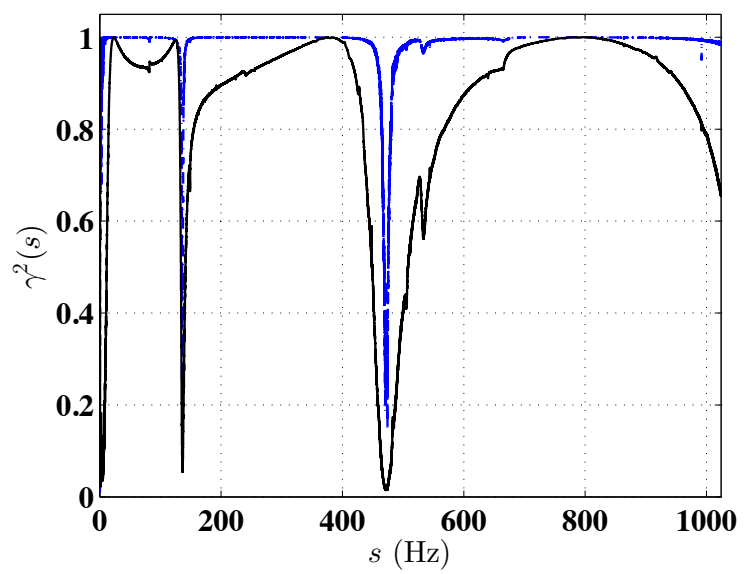

(b)

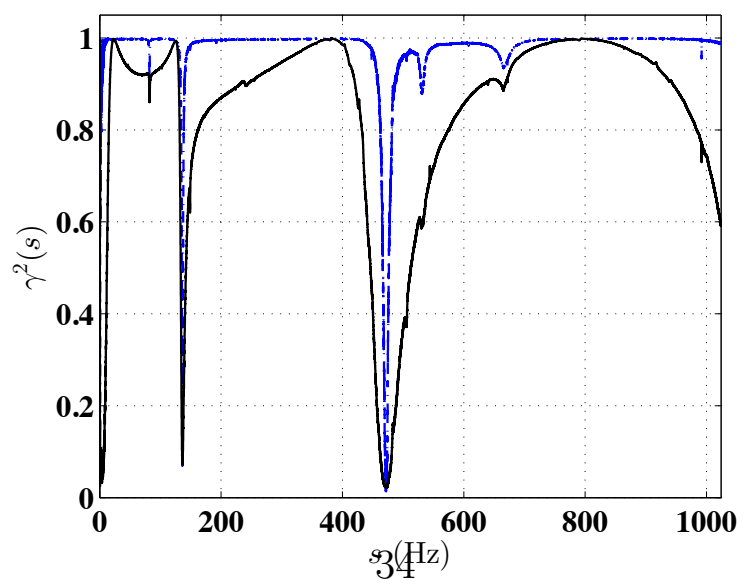

(c)

Figure 11: Comparisons of conventional (dashed line) and new (solid line) coherence functions using $\ddot{z}(t)$ and $f(t)$ of (a) the first sampling period, (b) the first two sampling periods and (c) the first five sampling periods. 


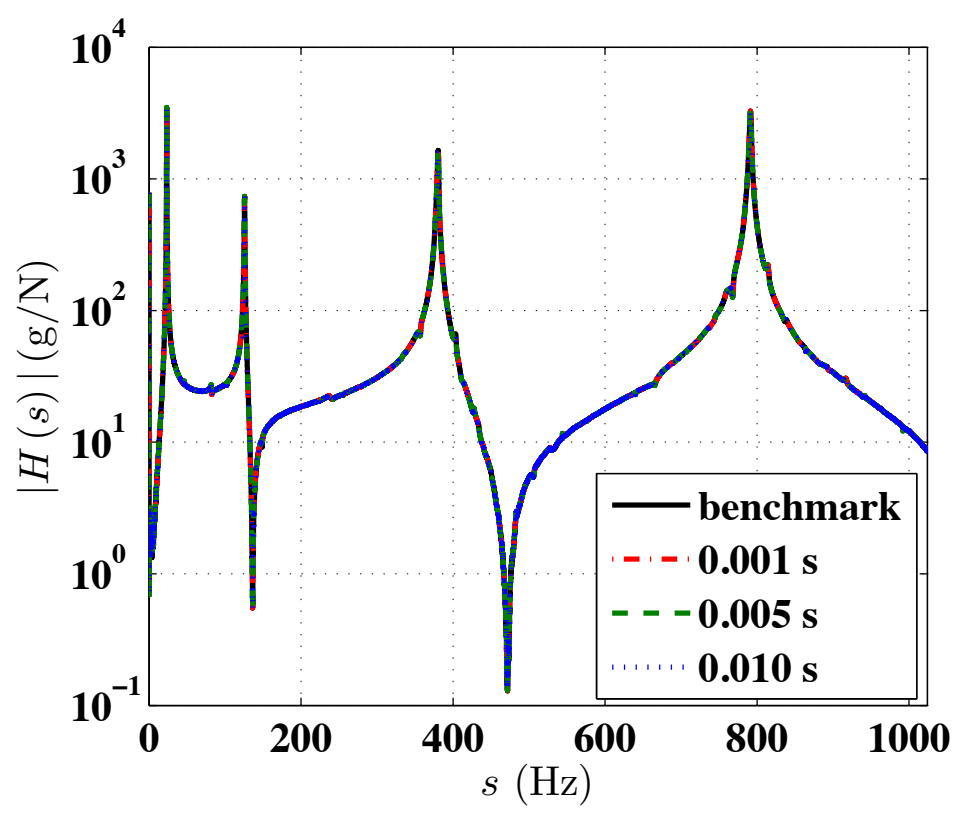

(a)

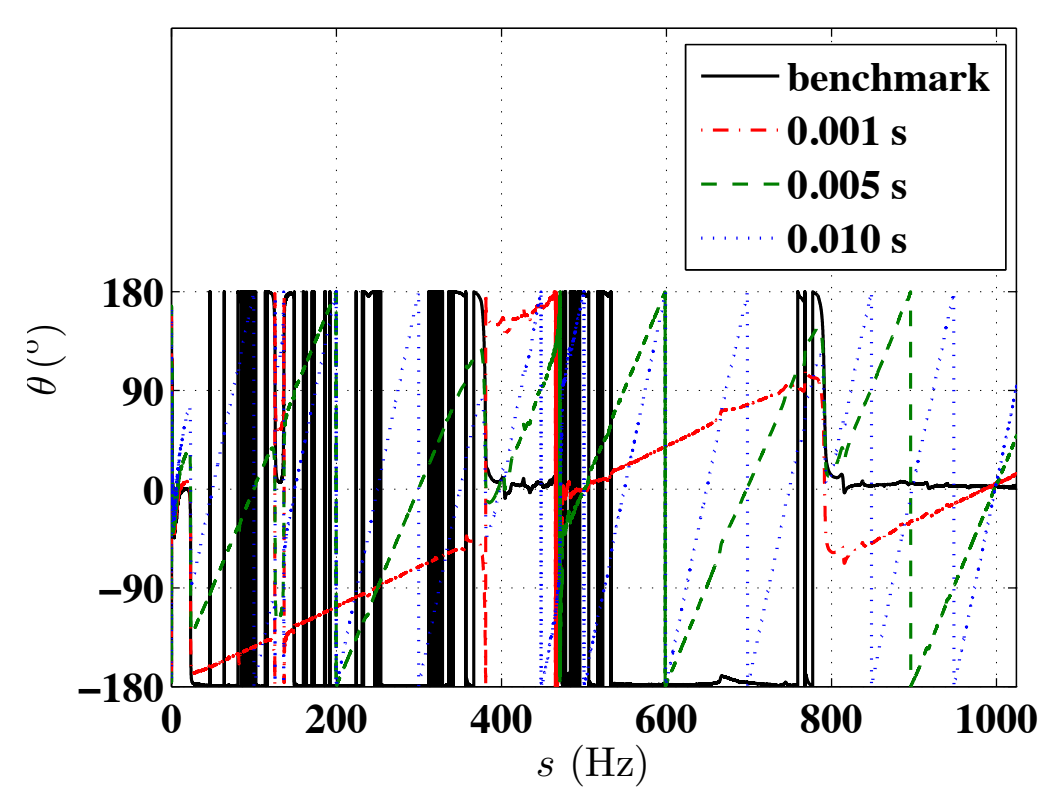

(b)

Figure 12: (a) Comparison of amplitudes of the benchmark FRF (benchmark) using $\ddot{z}(t)$ and $f(t)$ of the first five sampling periods in Fig. 9 and those using $\ddot{z}(t)$ and $f(t)$ padded with zero series that had lengths of $0.001 \mathrm{~s}, 0.005 \mathrm{~s}$ and $0.010 \mathrm{~s}$; and (b) comparison of their phases. 


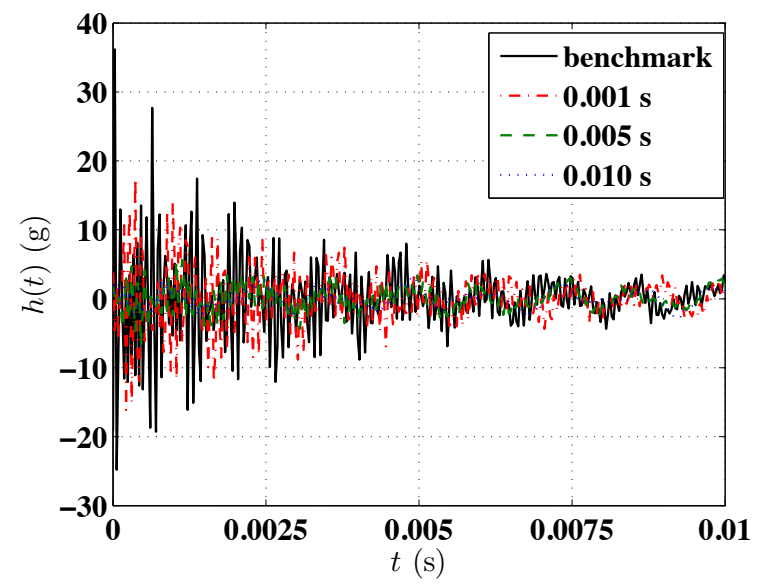

(a)

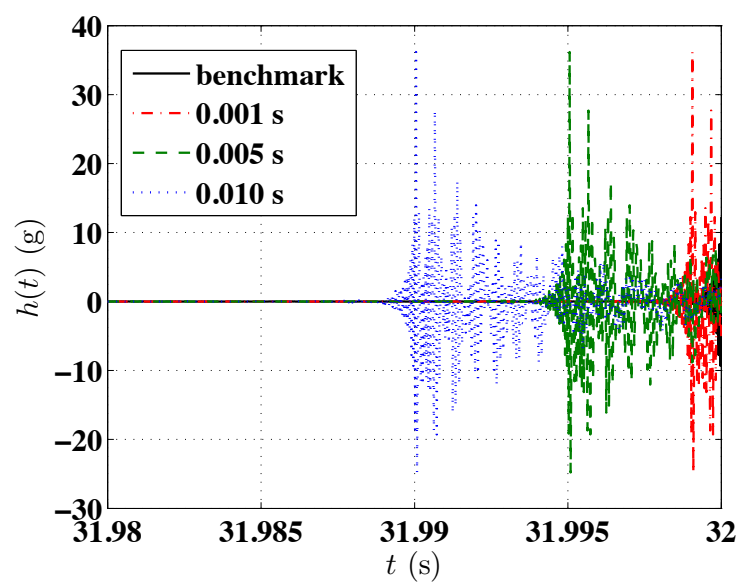

(b)

Figure 13: Comparison of IRFs associated with the benchmark FRF (benchmark) using $\ddot{z}(t)$ and $f(t)$ of the first five sampling periods in Fig. 9 and those using $\ddot{z}(t)$ and $f(t)$ padded with zero series that had lengths of $0.001 \mathrm{~s}, 0.005 \mathrm{~s}$ and $0.01 \mathrm{~s}$ in different time intervals: (a) between $t=0 \mathrm{~s}$ and $t=0.01 \mathrm{~s}$ and (b) between $t=31.98 \mathrm{~s}$ and $t=32 \mathrm{~s}$. 


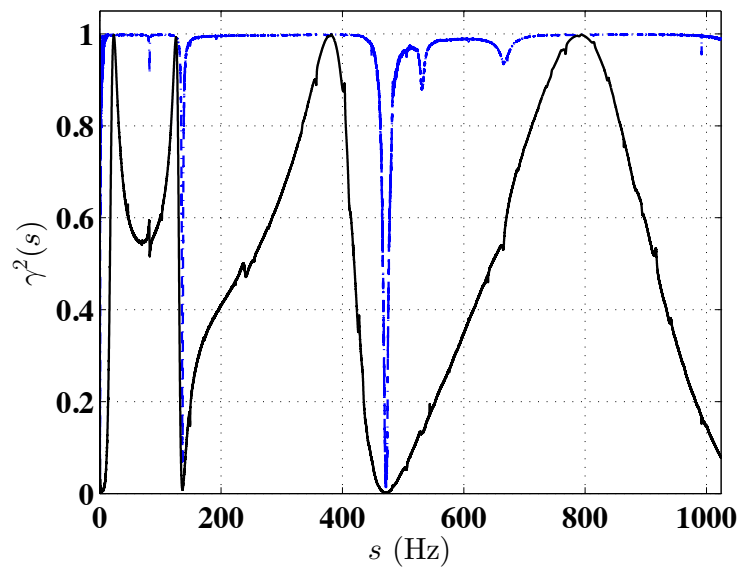

(a)

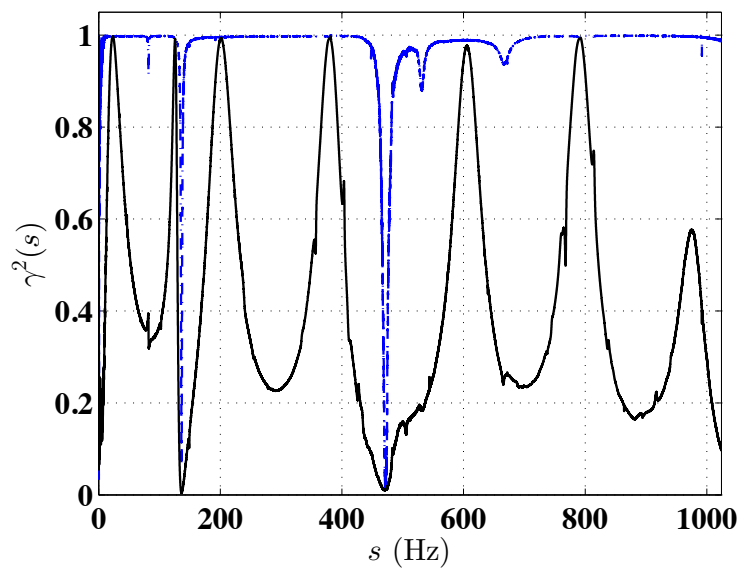

(b)

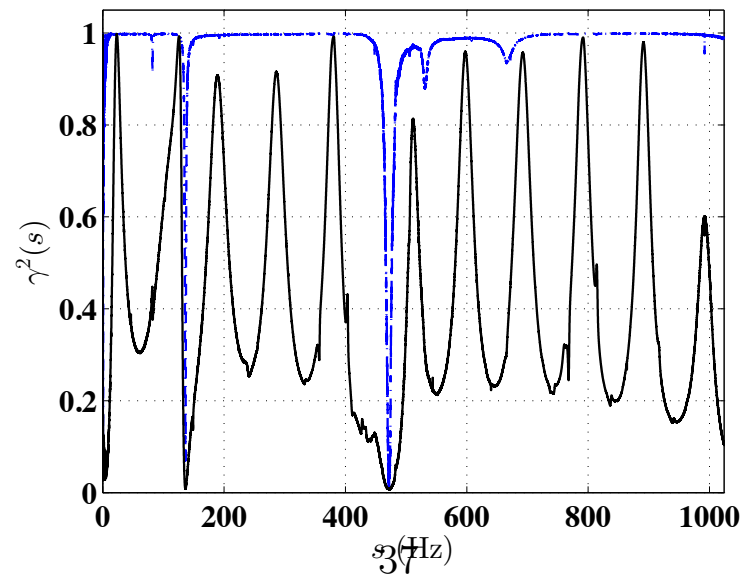

(c)

Figure 14: Comparisons of conventional and new coherence functions using $\ddot{z}(t)$ and $f(t)$ padded with zero series that had different lengths of: (a) $0.001 \mathrm{~s}$, (b) $0.005 \mathrm{~s}$ and (c) 0.01 S. 


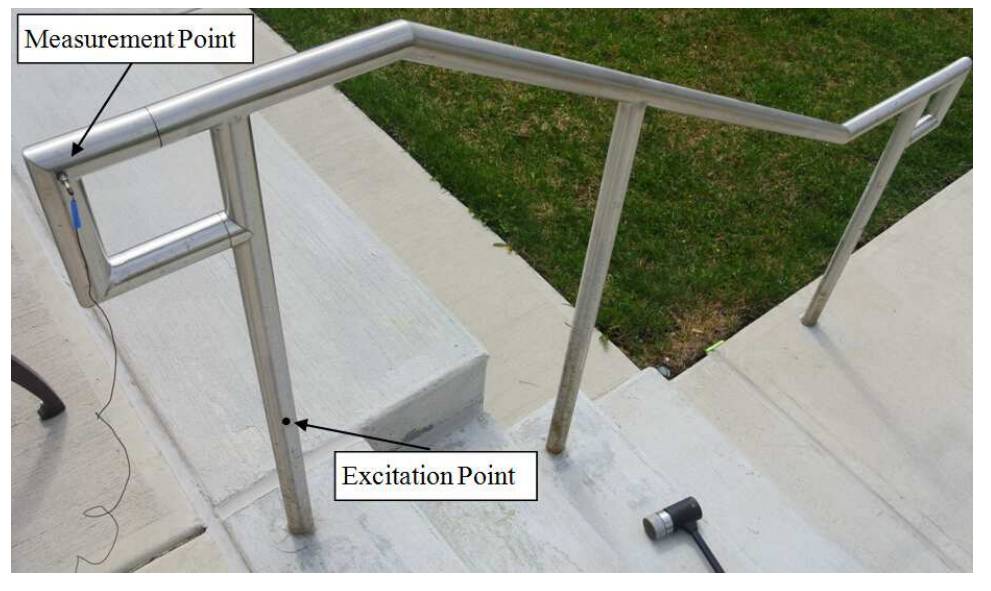

Figure 15: Test setup of an outdoor rail. 


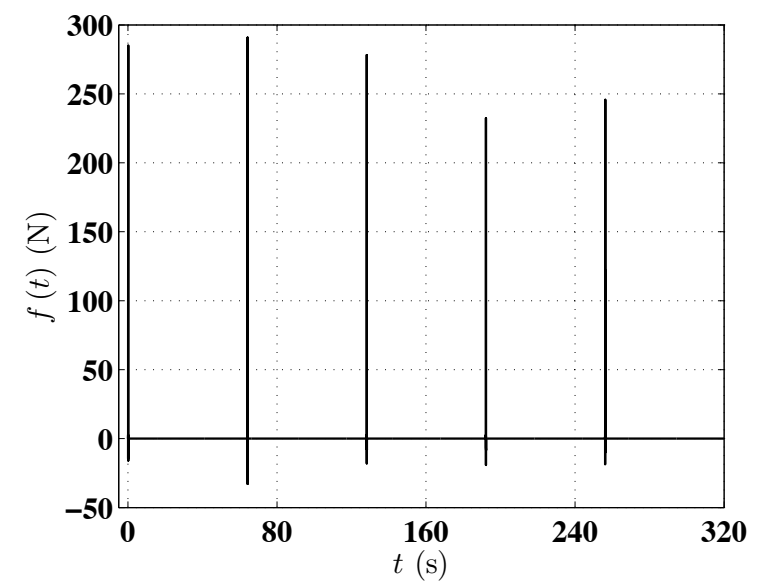

(a)

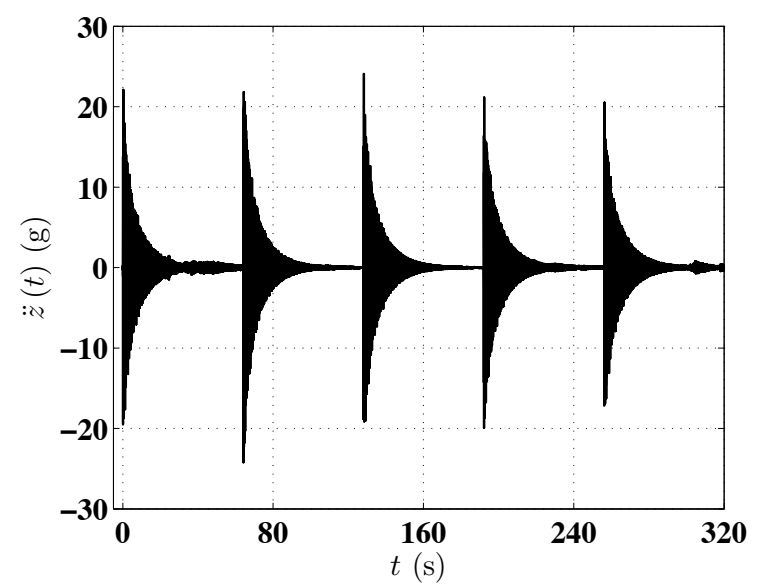

(b)

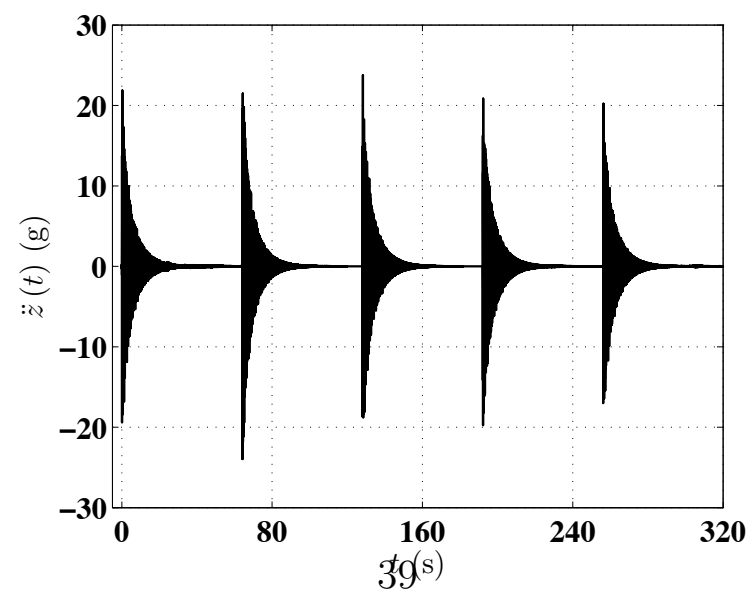

(c)

Figure 16: (a) Manually generated impacts at the excitation point on the rail in Fig. 15, (b) responses of the measurement point on the rail in Fig. 15 of the five sampling periods and (c) windowed responses of the measurement point. 


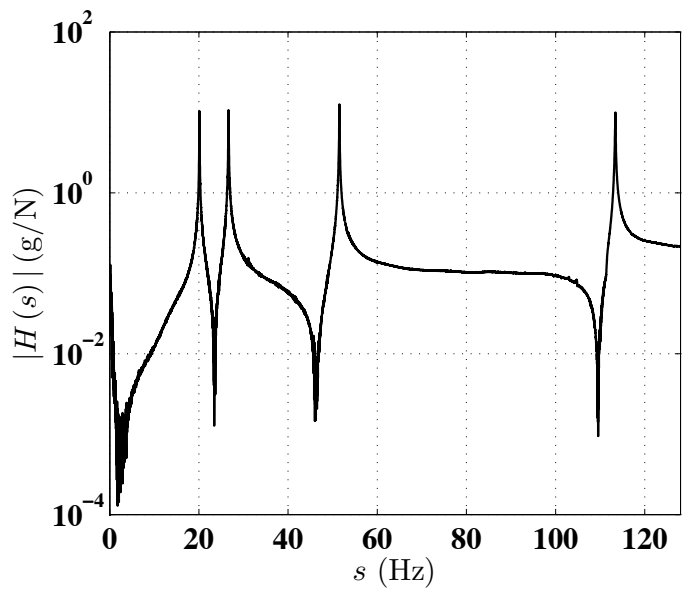

(a)

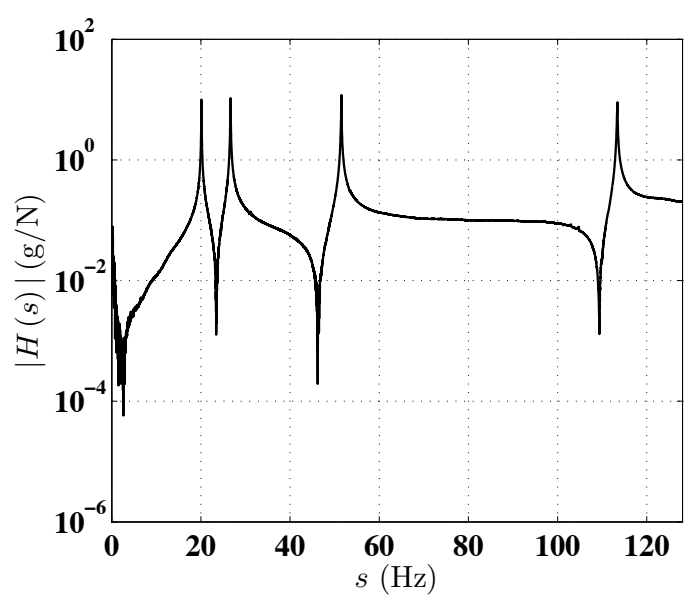

(c)

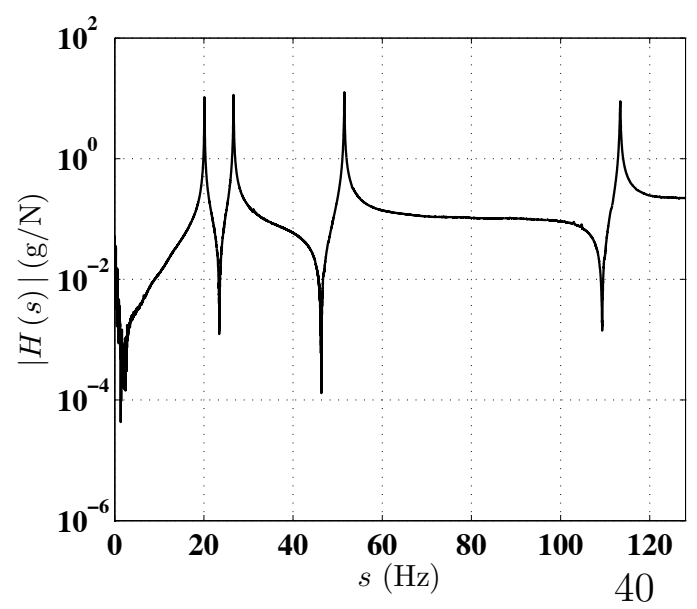

(e)

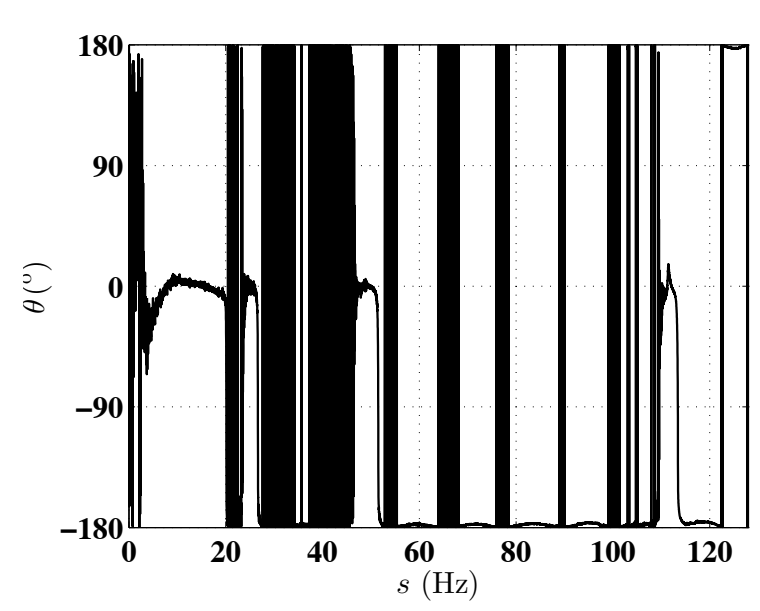

(b)

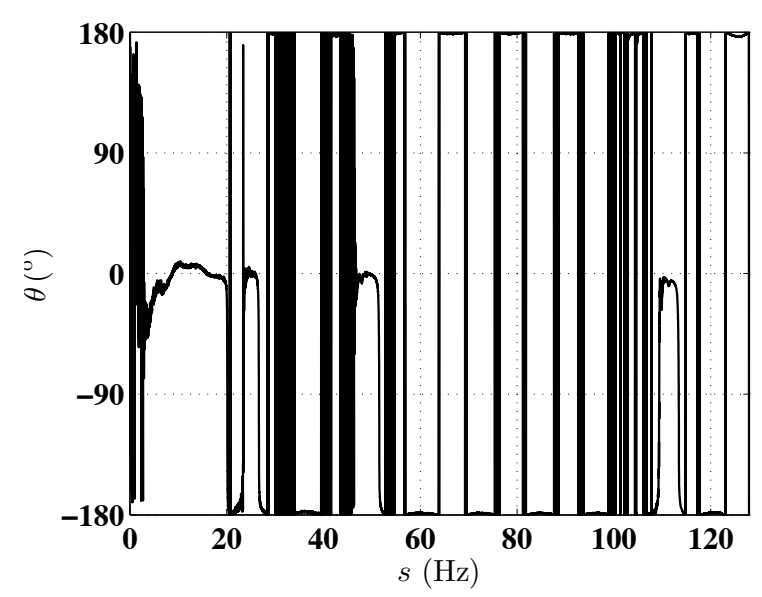

(d)

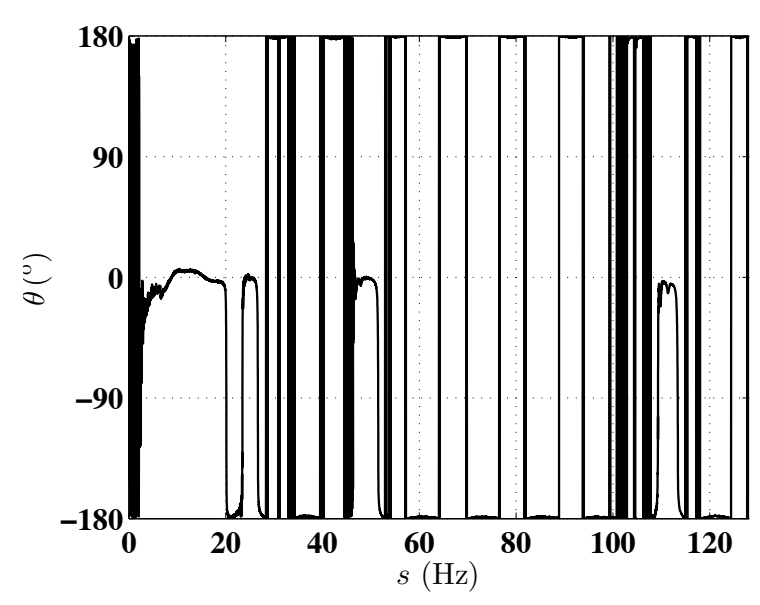

(f)

Figure 17: (a) Amplitude and (b) phase of the FRF using $\ddot{z}(t)$ and $f(t)$ of the first sampling period in Fig. 16, (c) the amplitude and (d) phase of the FRF using $\ddot{z}(t)$ and $f(t)$ of the first two sampling periods in Fig. 16, (e) the amplitude and (f) phase of the FRF using $\ddot{z}(t)$ and $f(t)$ of the first five sampling periods in Fig. 16. 


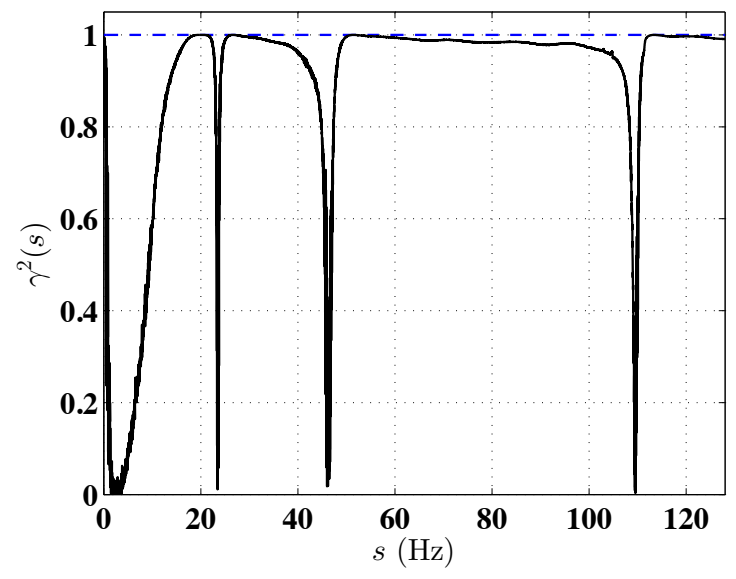

(a)

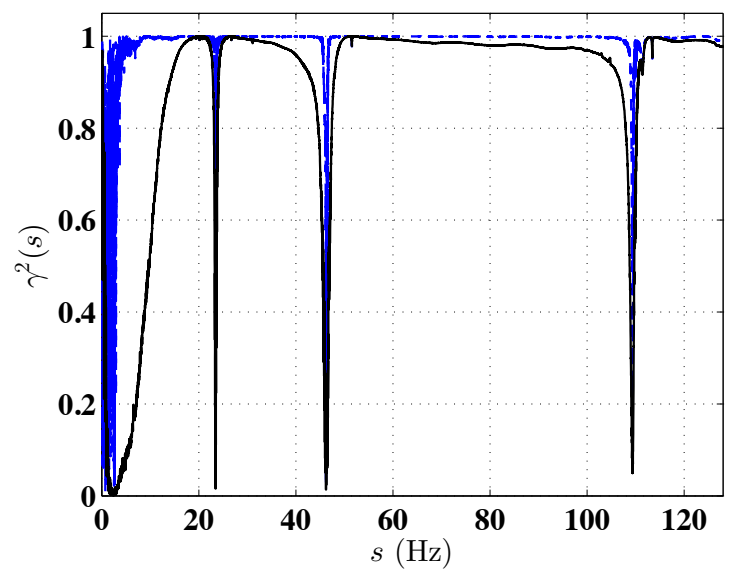

(b)

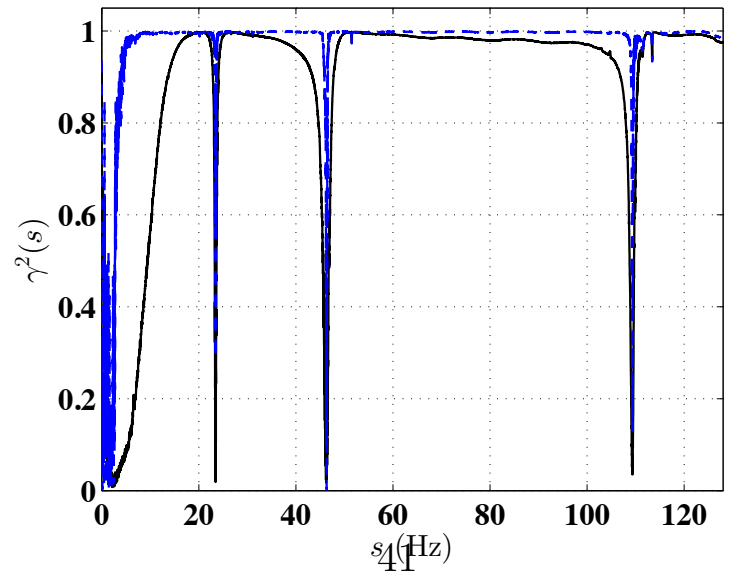

(c)

Figure 18: Comparisons of conventional (dashed line) and new (solid line) coherence functions using $\ddot{z}(t)$ and $f(t)$ of (a) the first sampling period, (b) the first two sampling periods and (c) the first five sampling periods. 


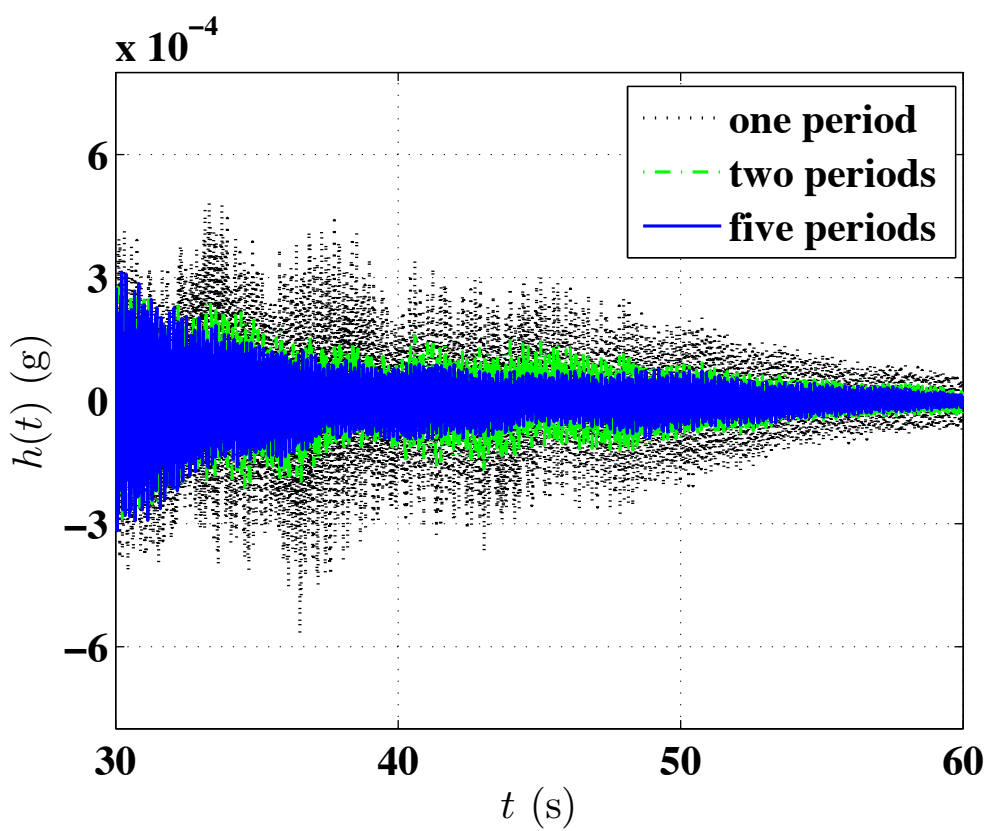

Figure 19: Comparison of IRFs associated with the FRFs between $t=30 \mathrm{~s}$ and $t=60 \mathrm{~s}$ using $\ddot{z}(t)$ and $f(t)$ of the first, first two and first five sampling periods. 


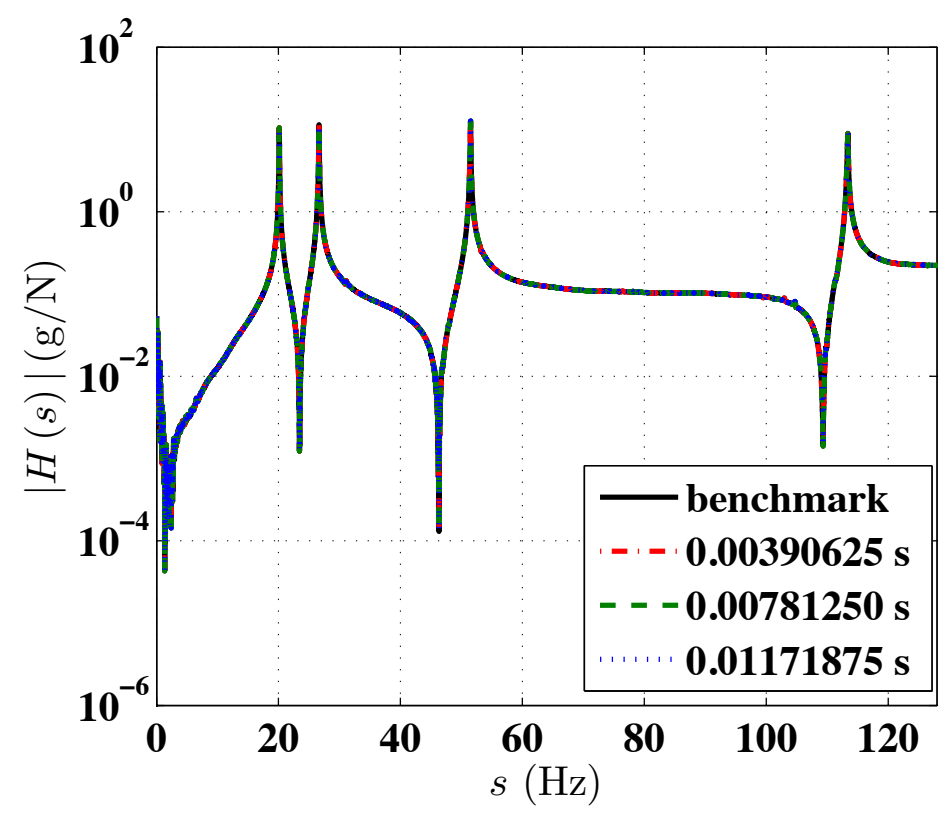

(a)

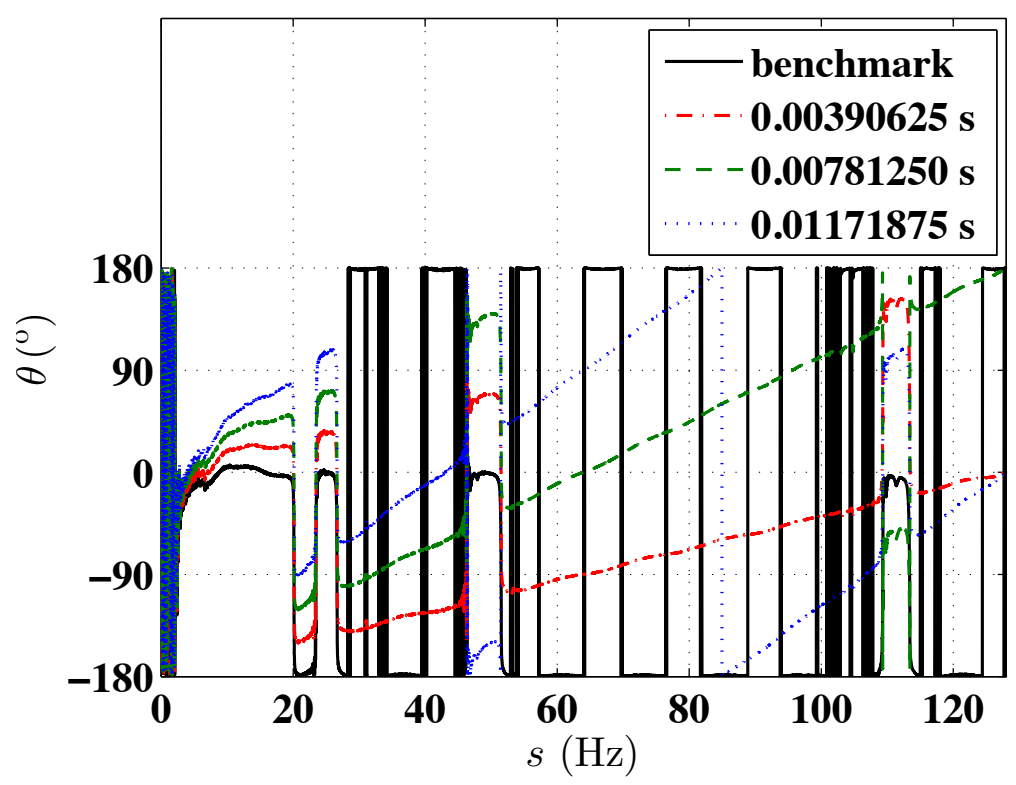

(b)

Figure 20: (a) Comparison of amplitudes of the benchmark FRF (benchmark) using $\ddot{z}(t)$ and $f(t)$ of the first five sampling periods in Fig. 16 and those using $\ddot{z}(t)$ and $f(t)$ padded with zero series that had lengths of $0.00390625 \mathrm{~s}, 0.00781250 \mathrm{~s}$ and $0.01171815 \mathrm{~s}$; and (b) comparison of their phases. 


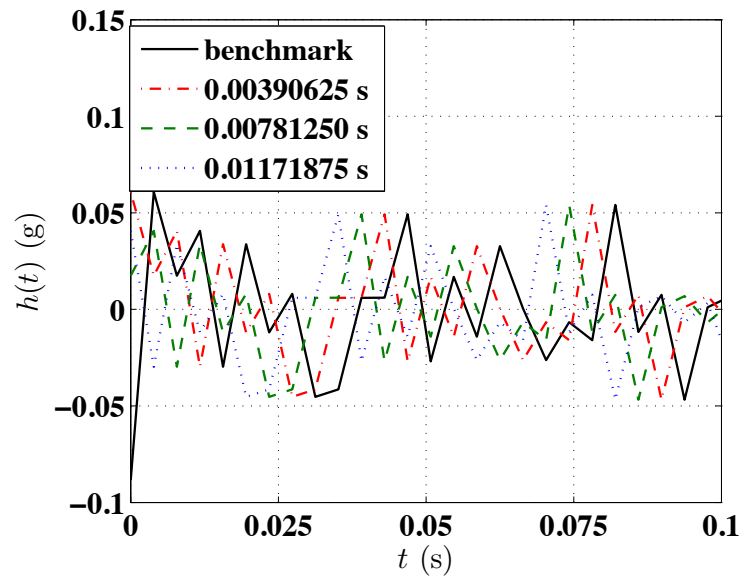

(a)

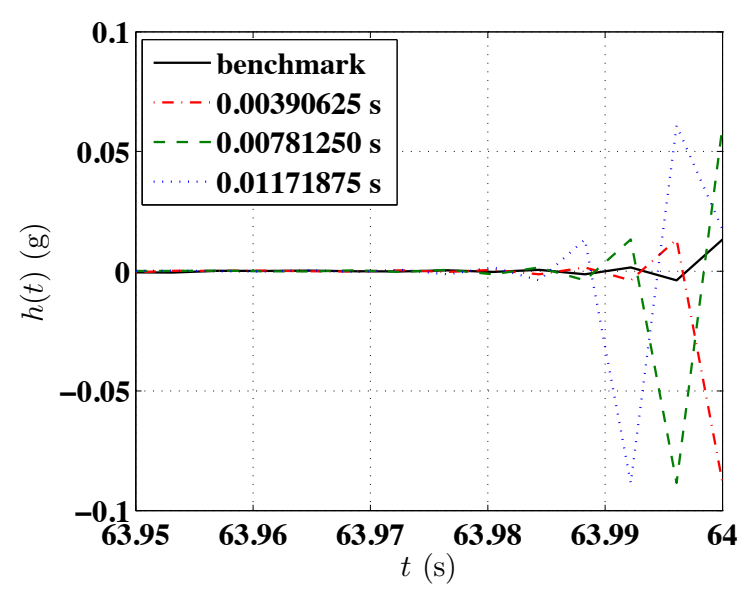

(b)

Figure 21: Comparison of IRFs associated with the benchmark FRF (benchmark) using $\ddot{z}(t)$ and $f(t)$ of the first five sampling periods in Fig. 16 and those using $\ddot{z}(t)$ and $f(t)$ padded with zero series that had lengths of $0.00390625 \mathrm{~s}, 0.00781250 \mathrm{~s}$ and $0.01171815 \mathrm{~s}$ in different time intervals: (a) between $t=0 \mathrm{~s}$ and $t=0.1 \mathrm{~s}$ and (b) between $t=63.95 \mathrm{~s}$ and $t=64 \mathrm{~s}$. 


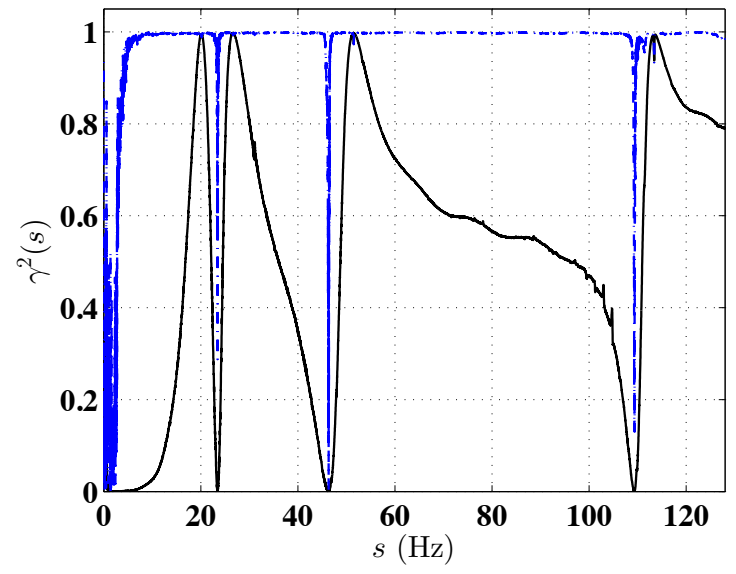

(a)

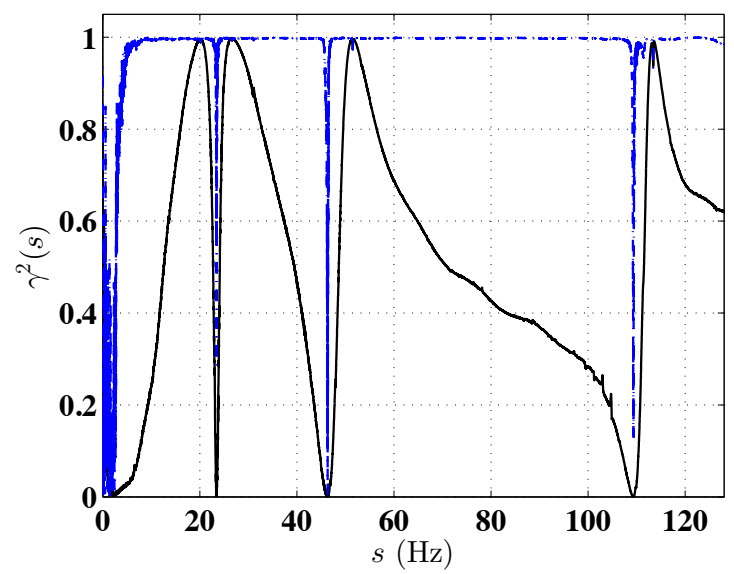

(b)

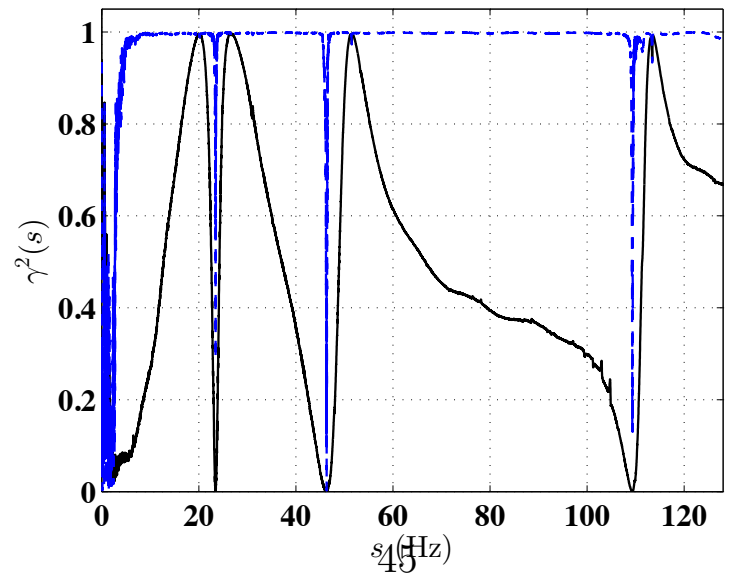

(c)

Figure 22: Comparisons of conventional (dashed line) and new (solid line) coherence functions using $\ddot{z}(t)$ and $f(t)$ padded with zero series that had different lengths of: (a) $0.00390625 \mathrm{~s}$, (b) $0.00781250 \mathrm{~s}$ and (c) $0.01171815 \mathrm{~s}$. 


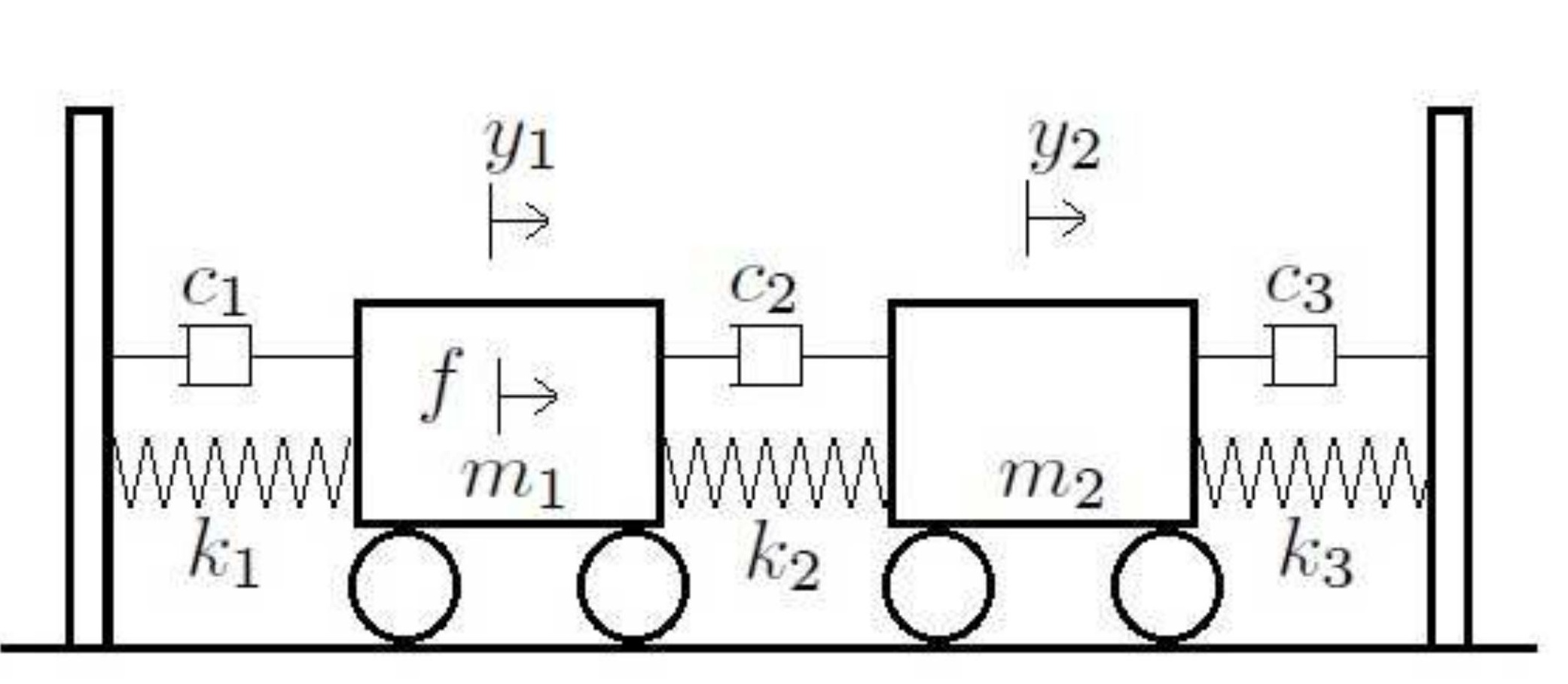

Figure 1

Figure 1 


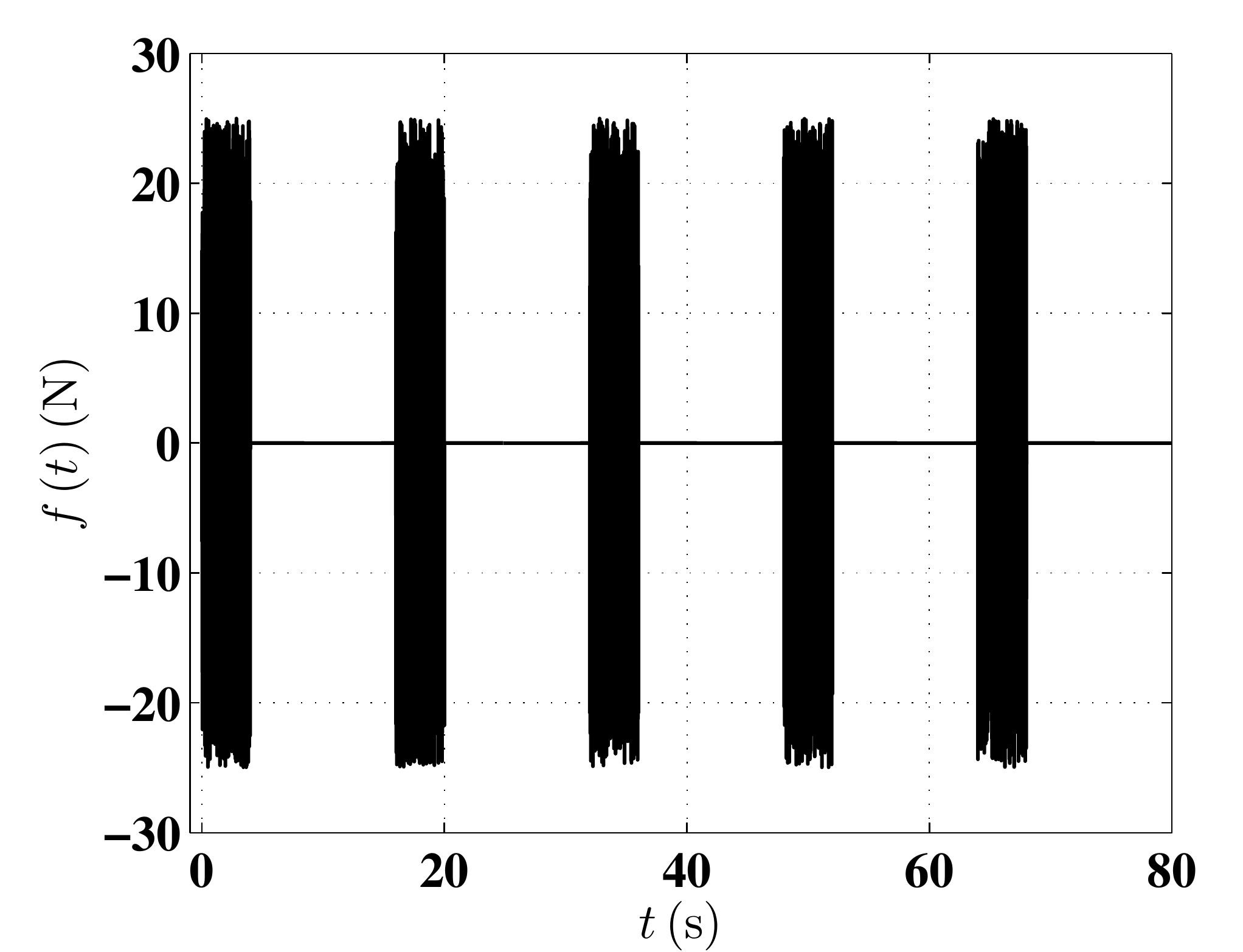

Figure 2a

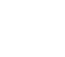
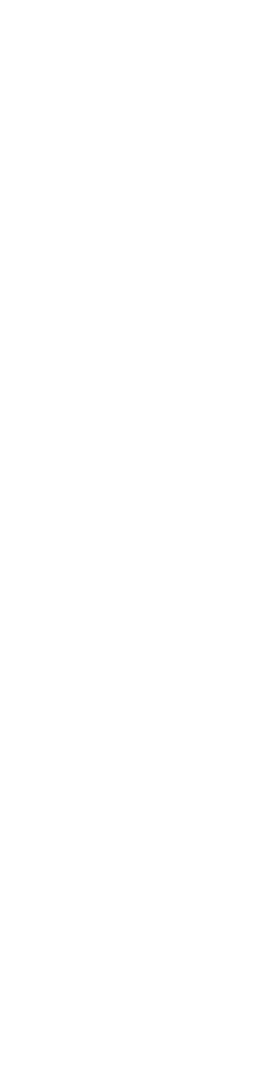

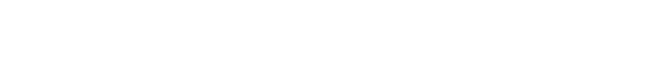

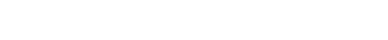

(1)

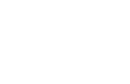

政

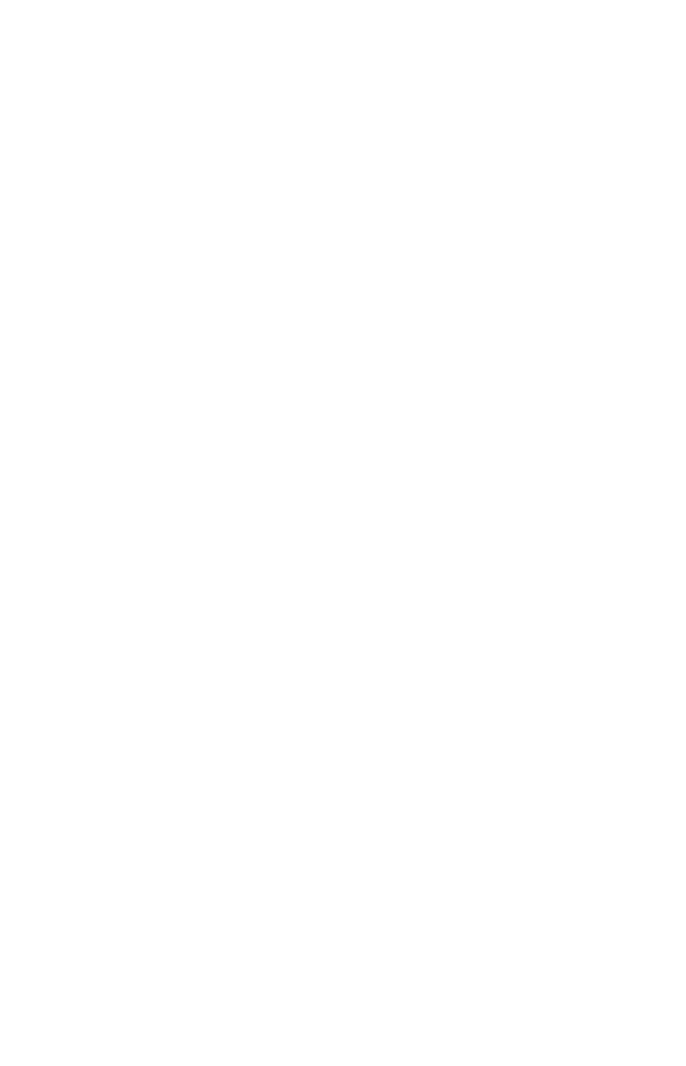

(1)

-

$\sqrt{2}-2-2$
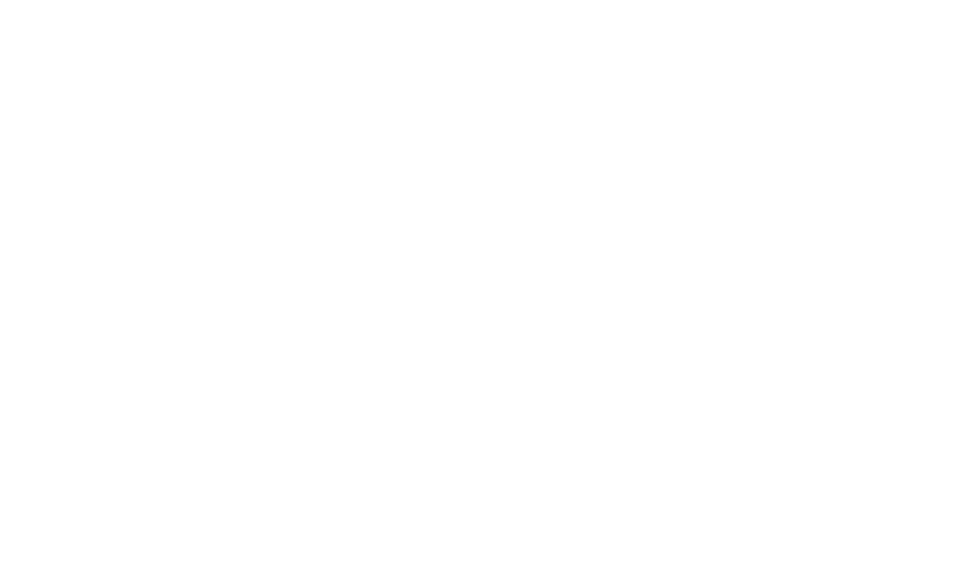
Figure $2 b$

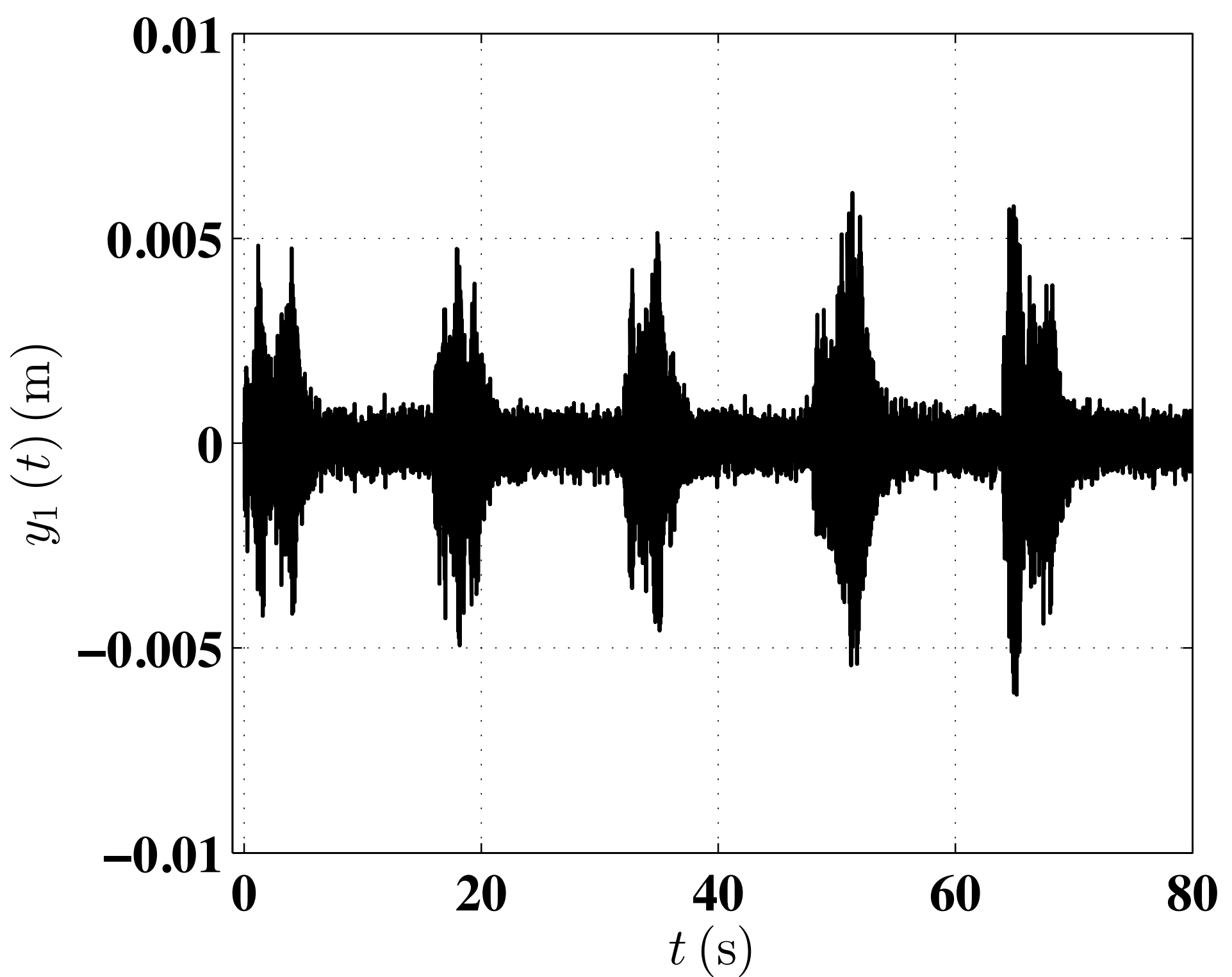




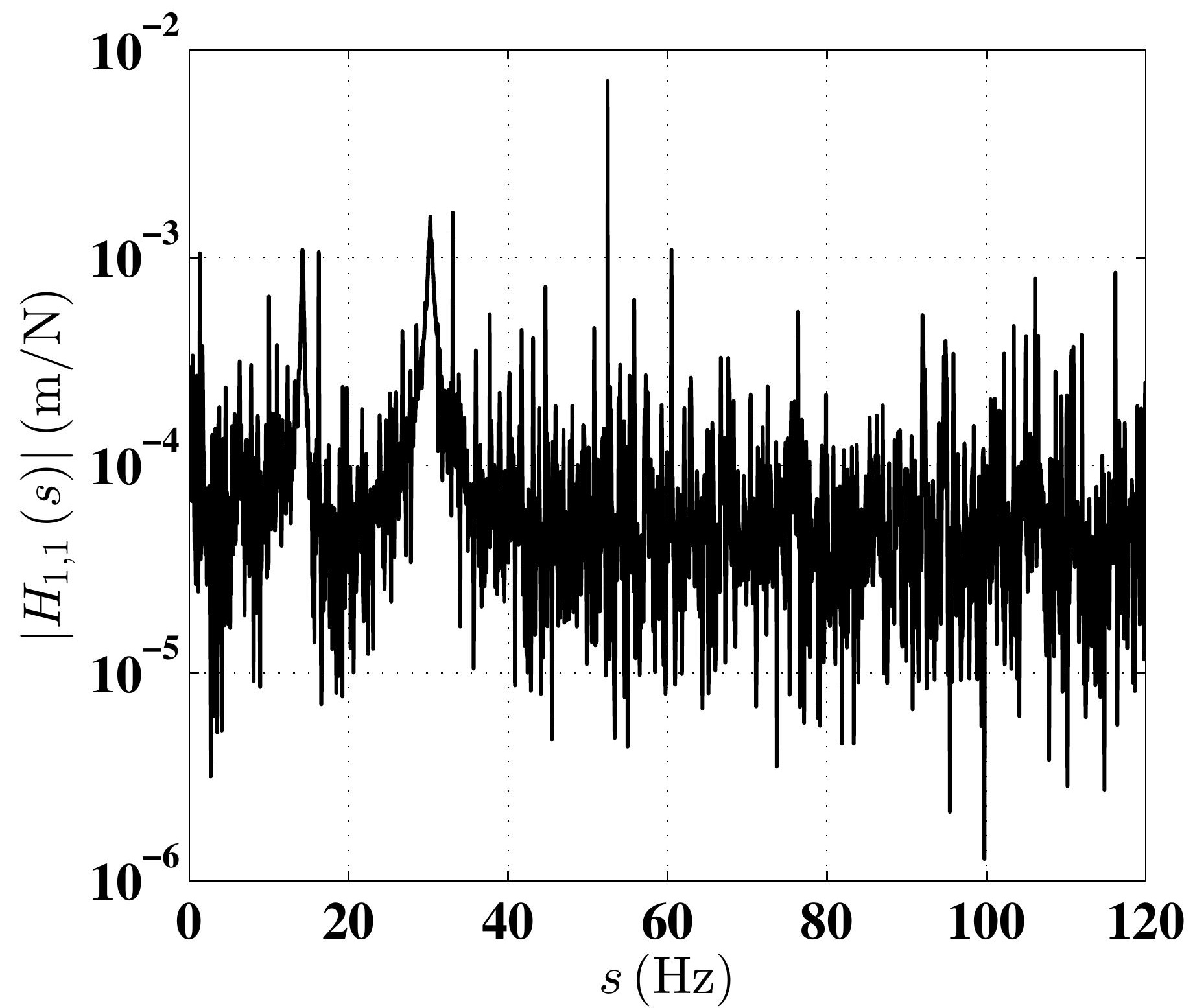




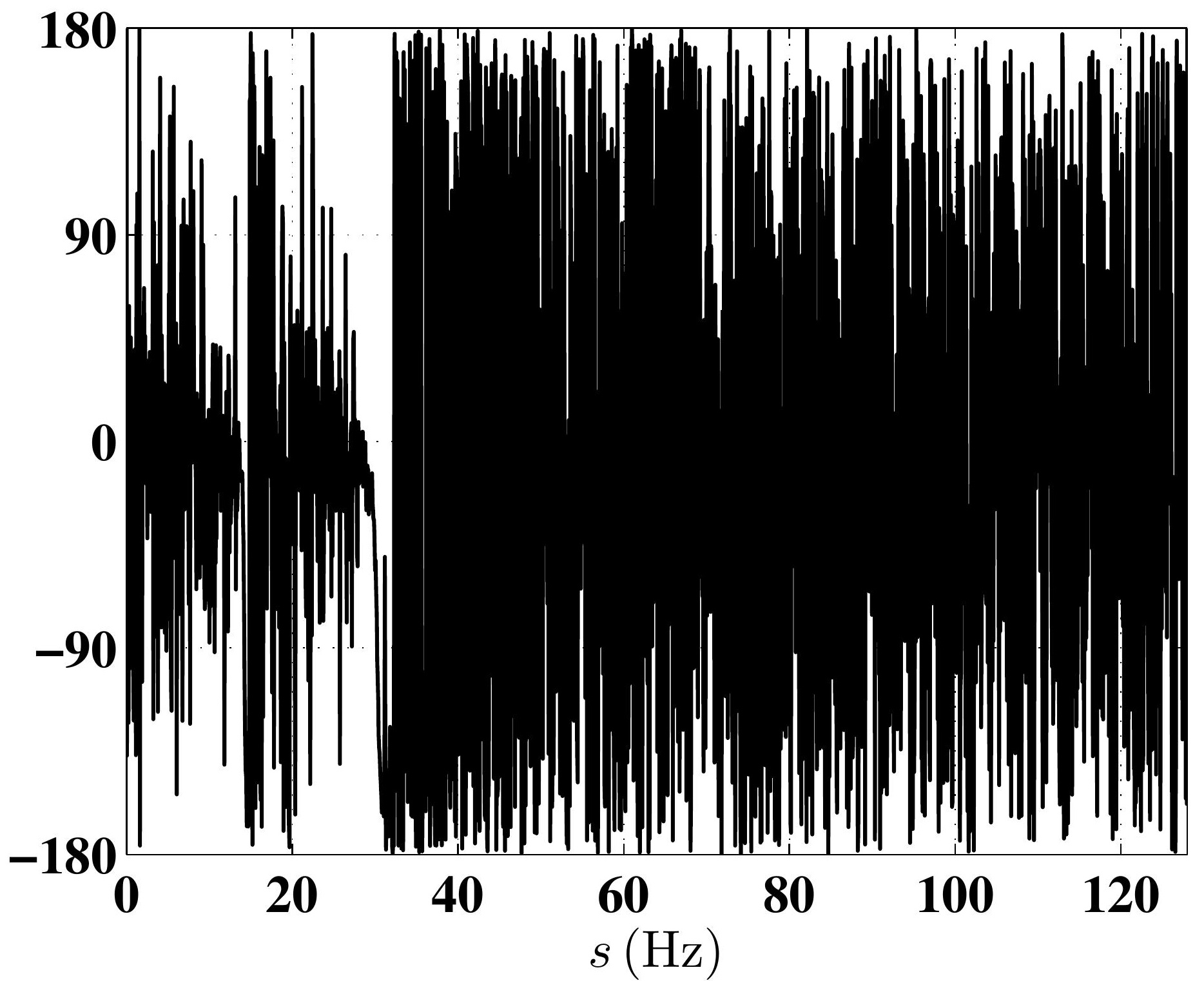




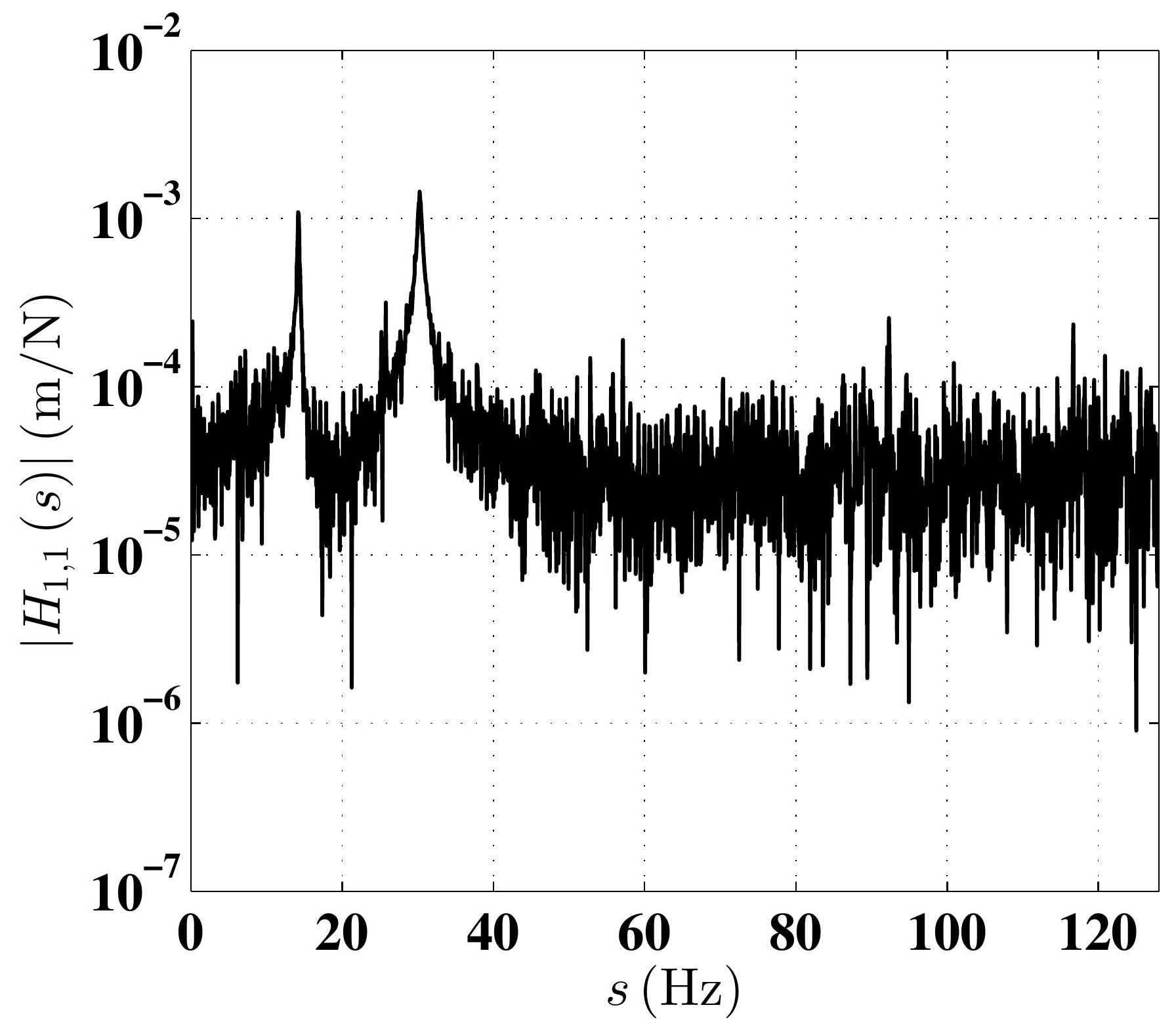




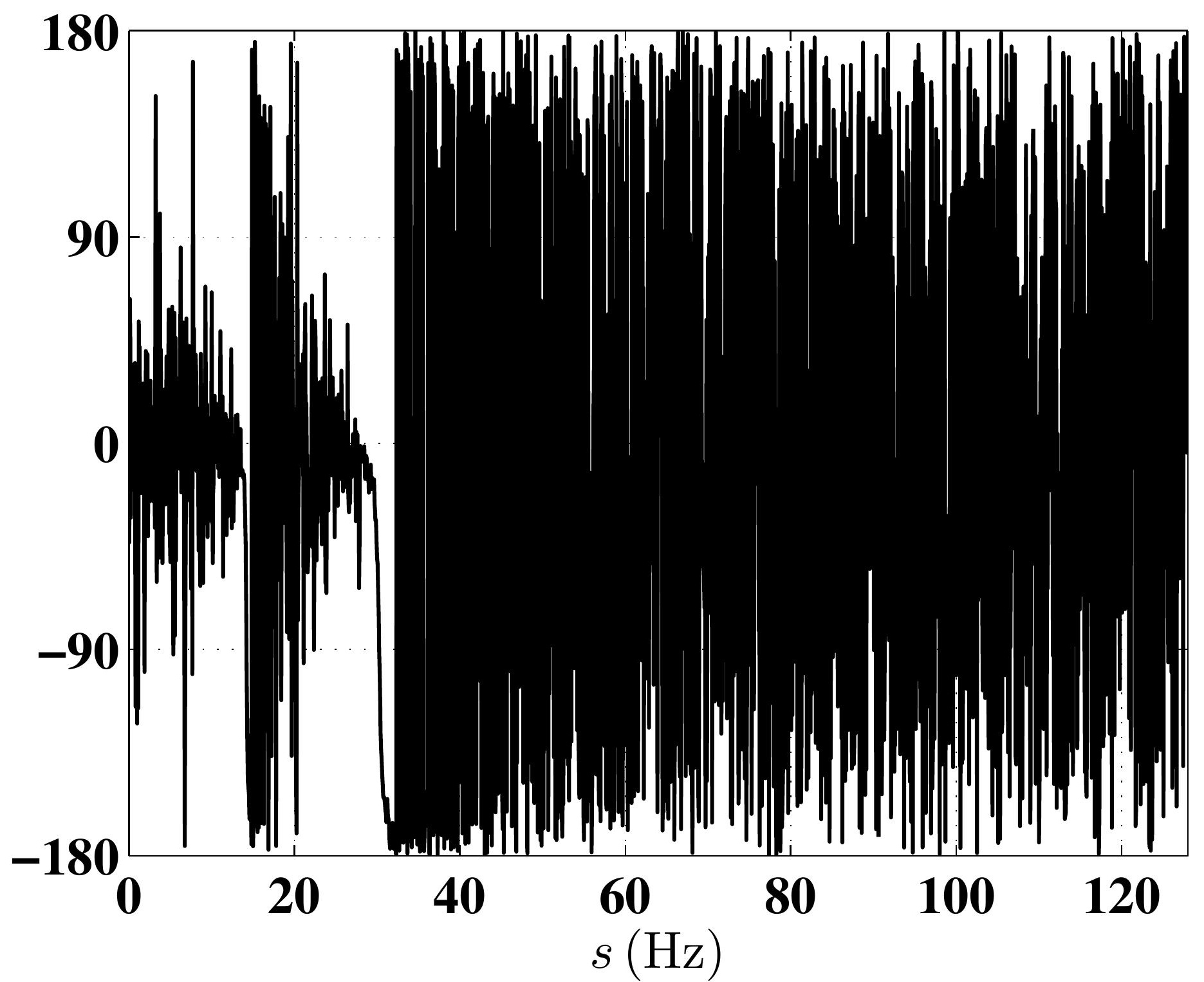




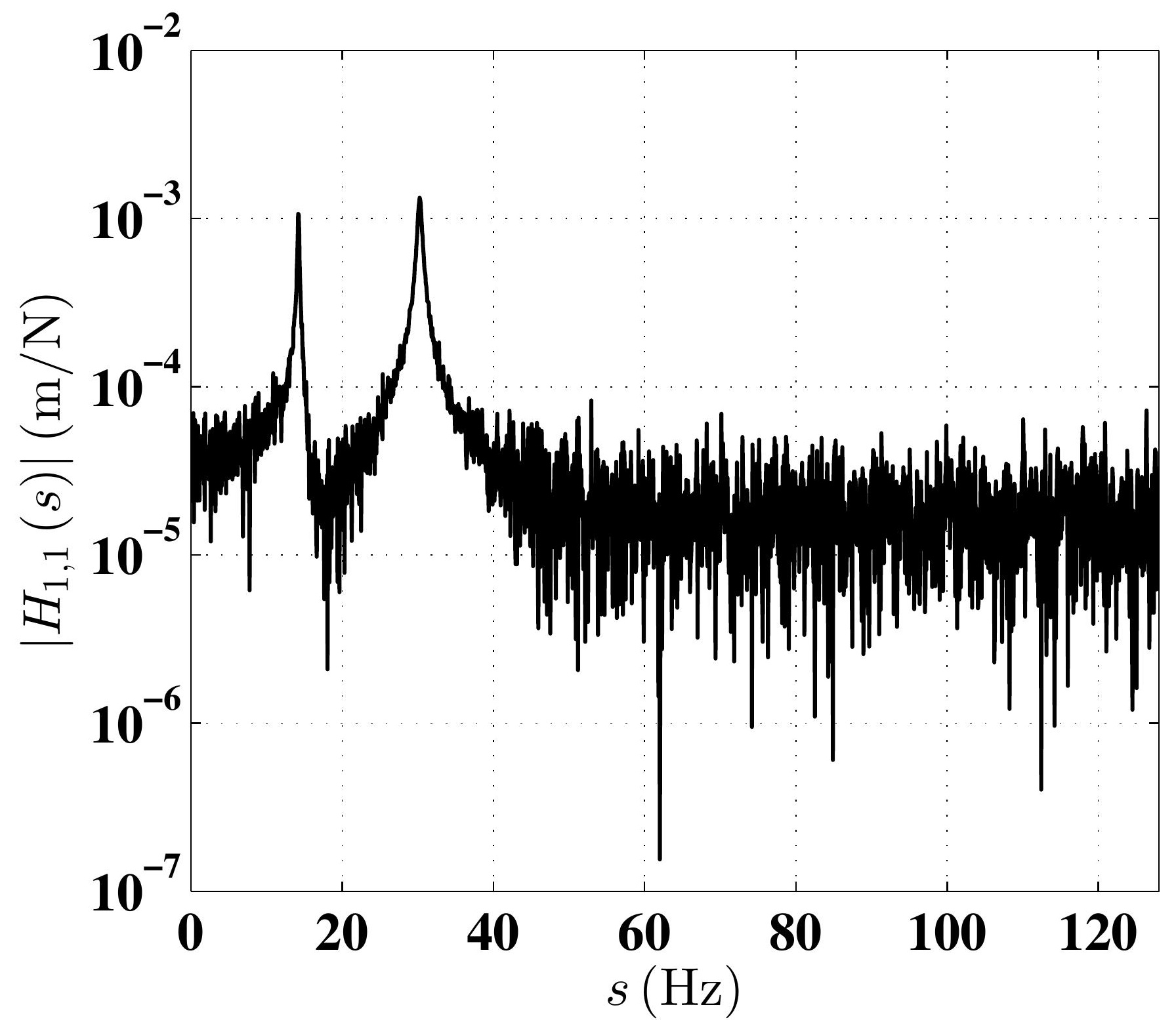




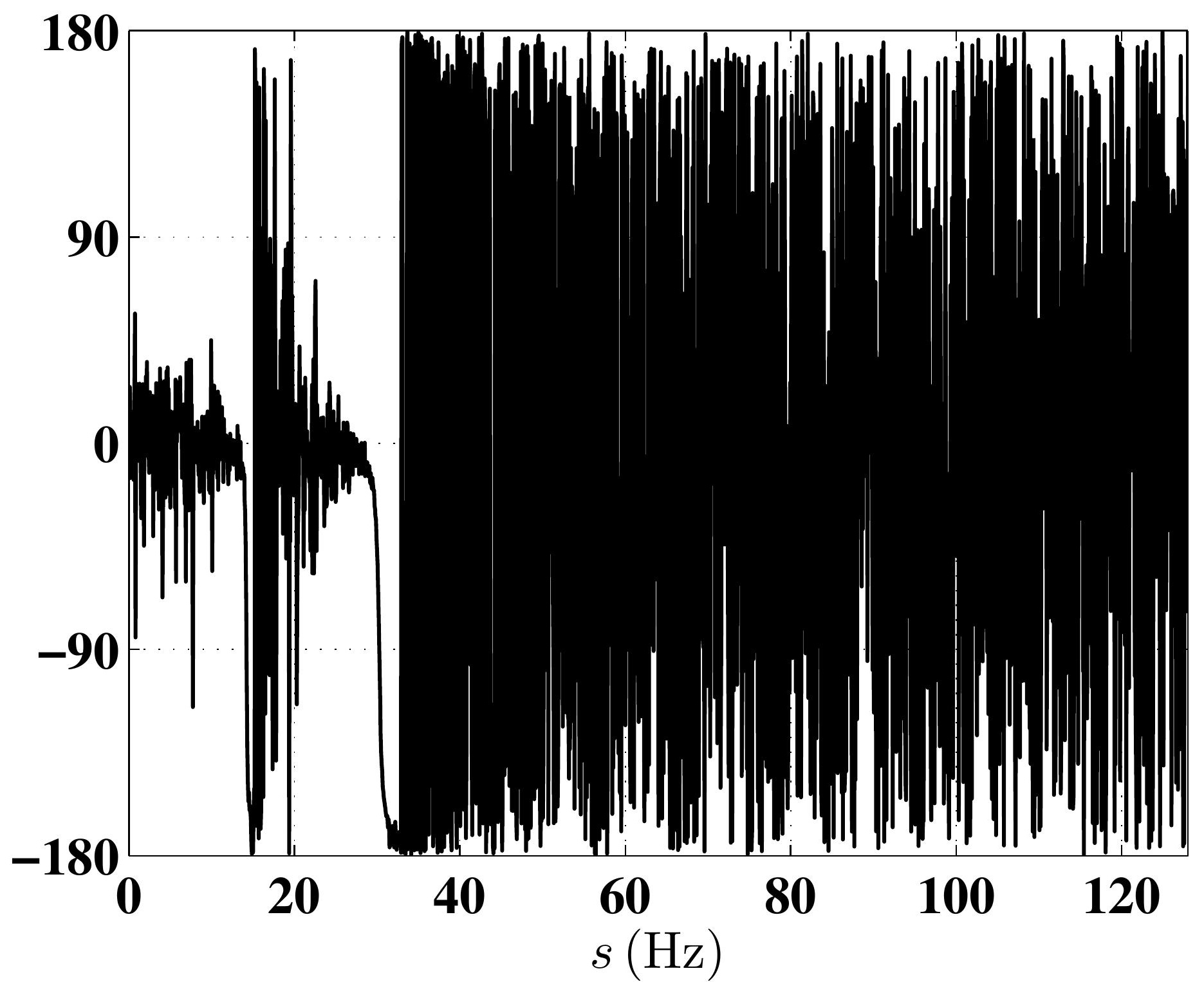




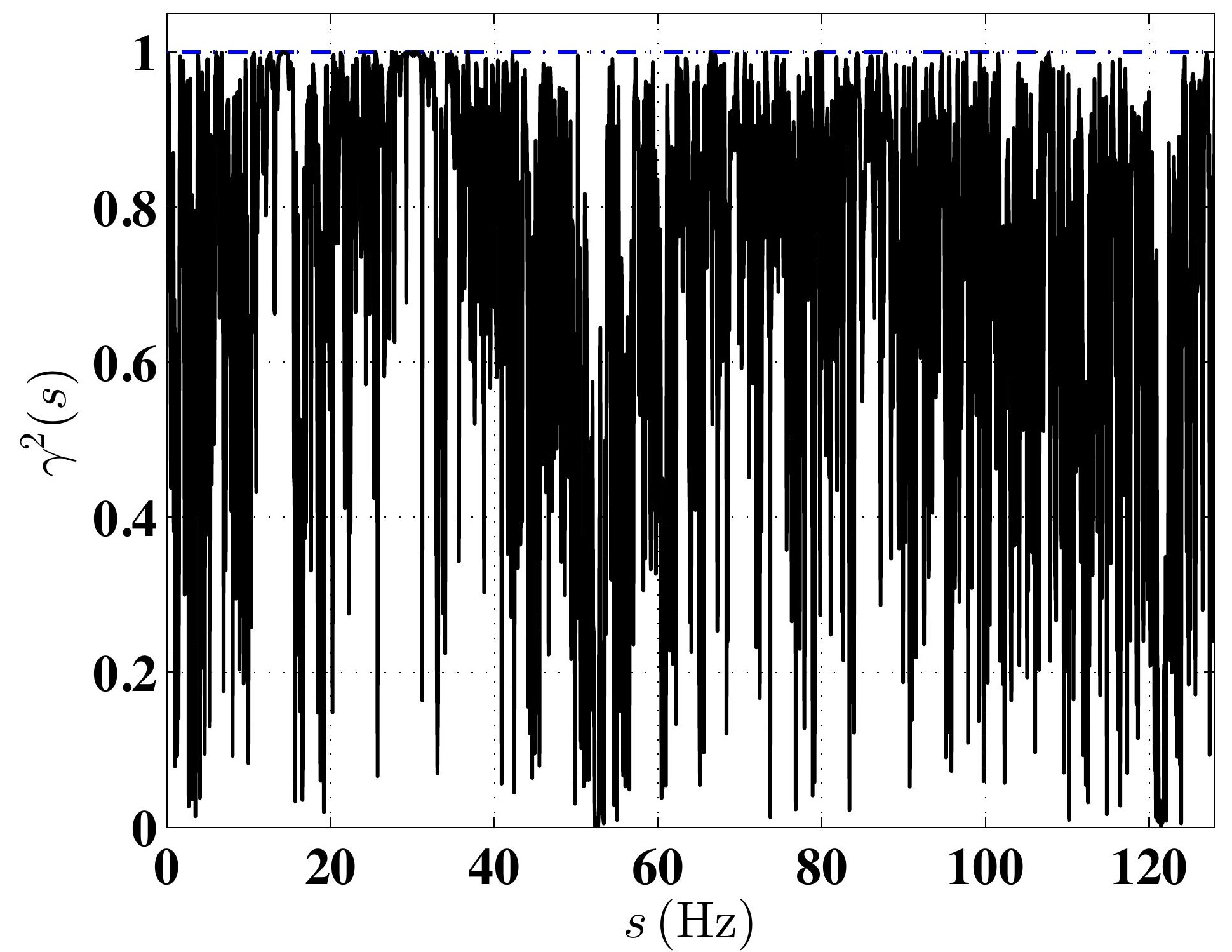




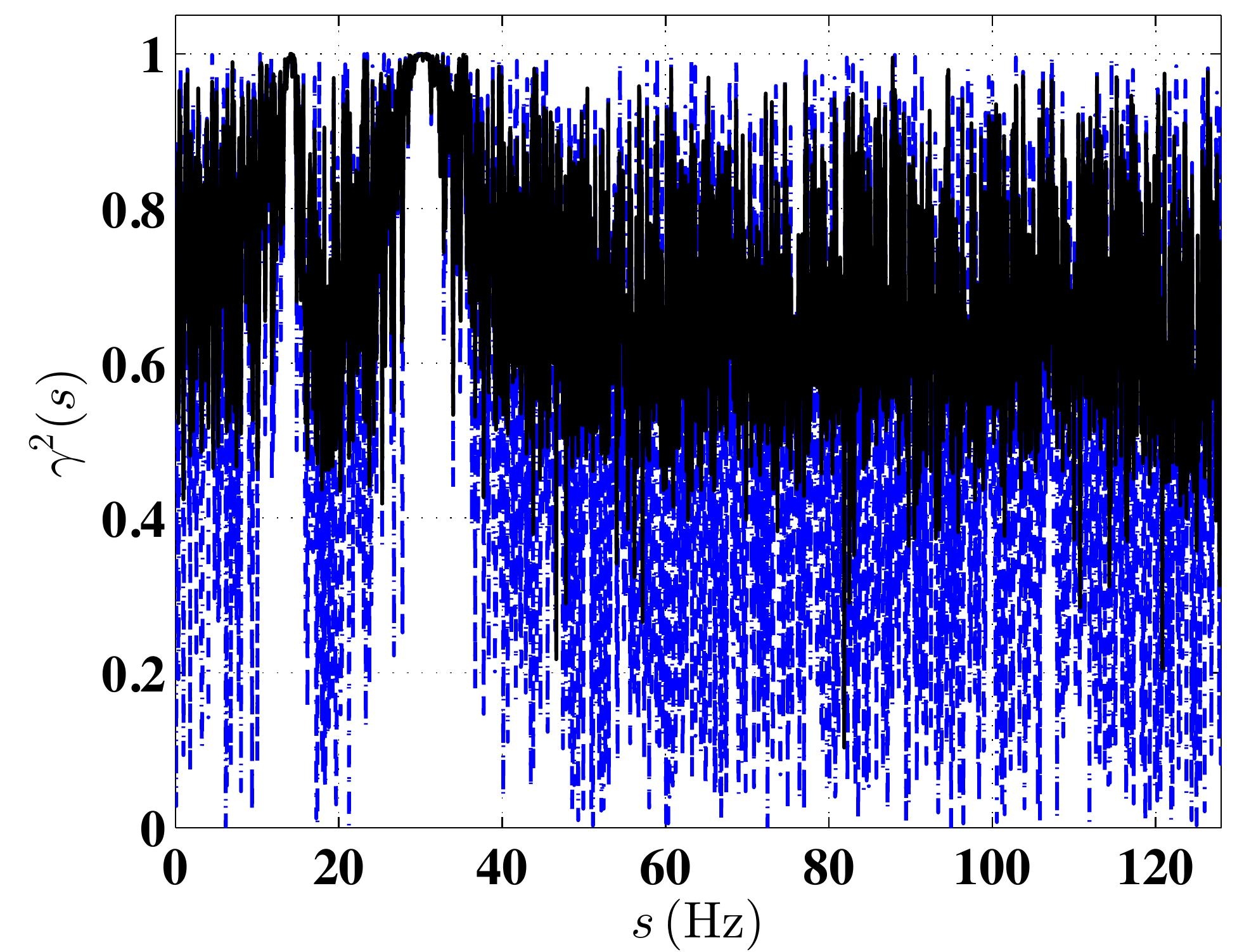




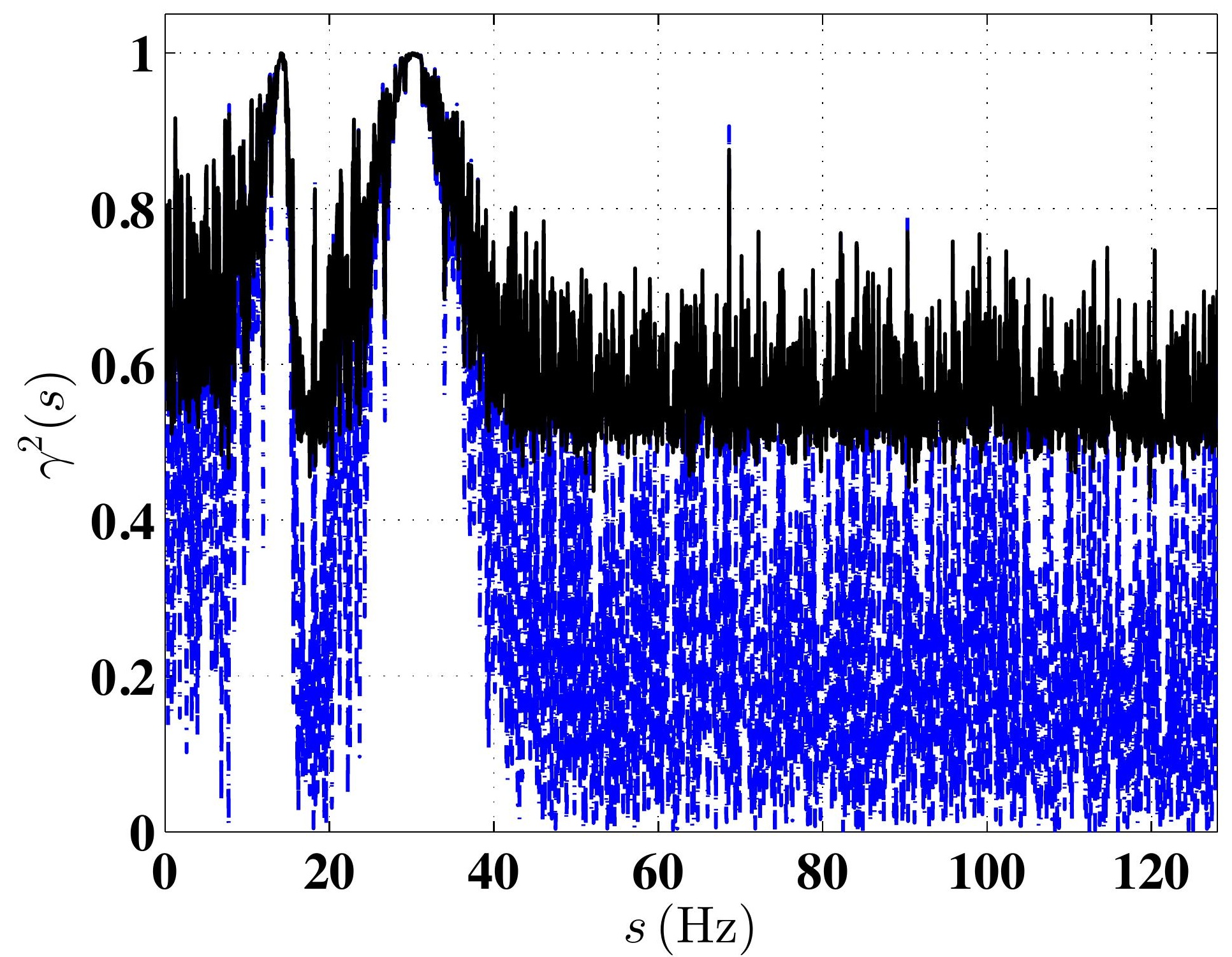




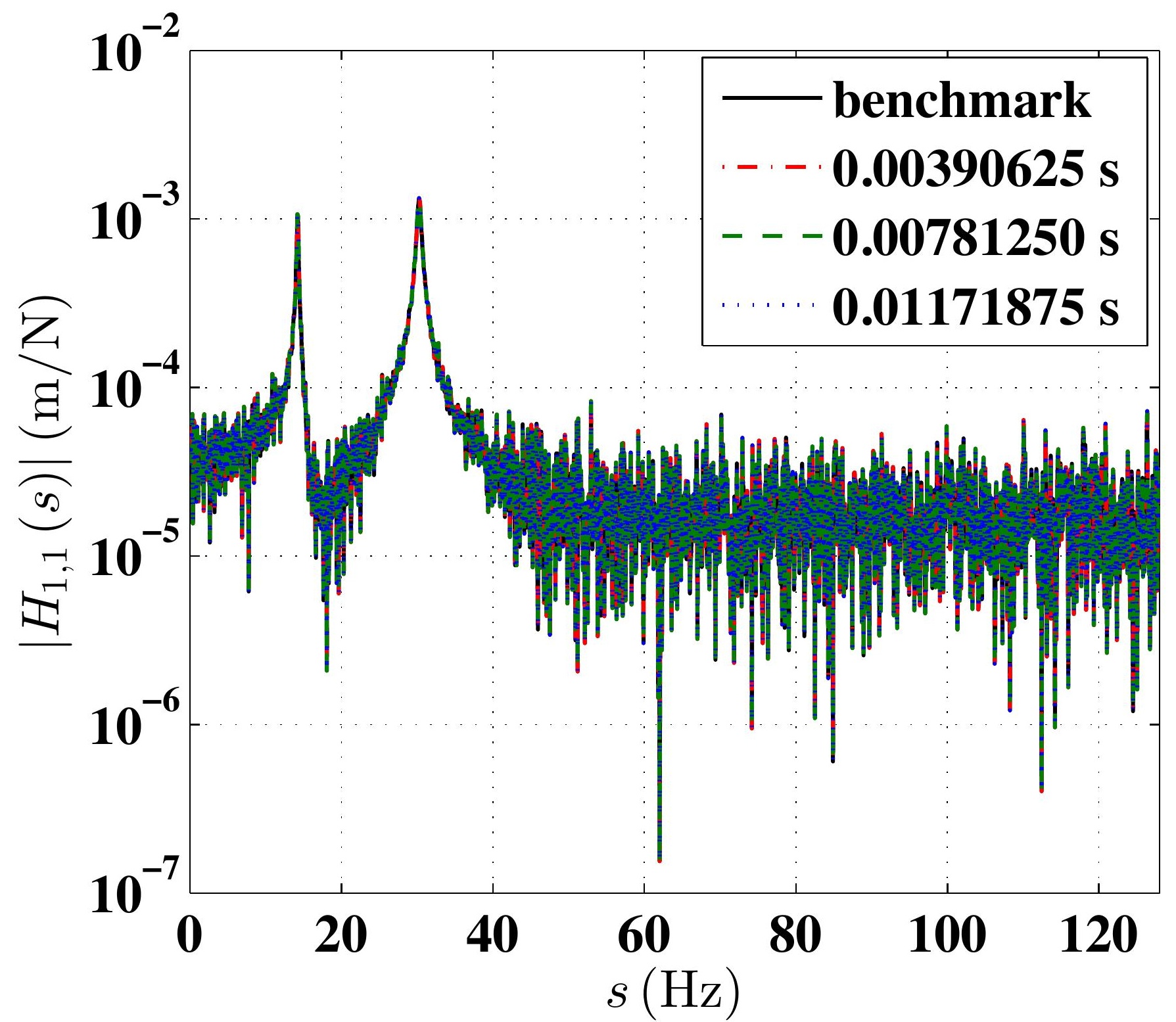




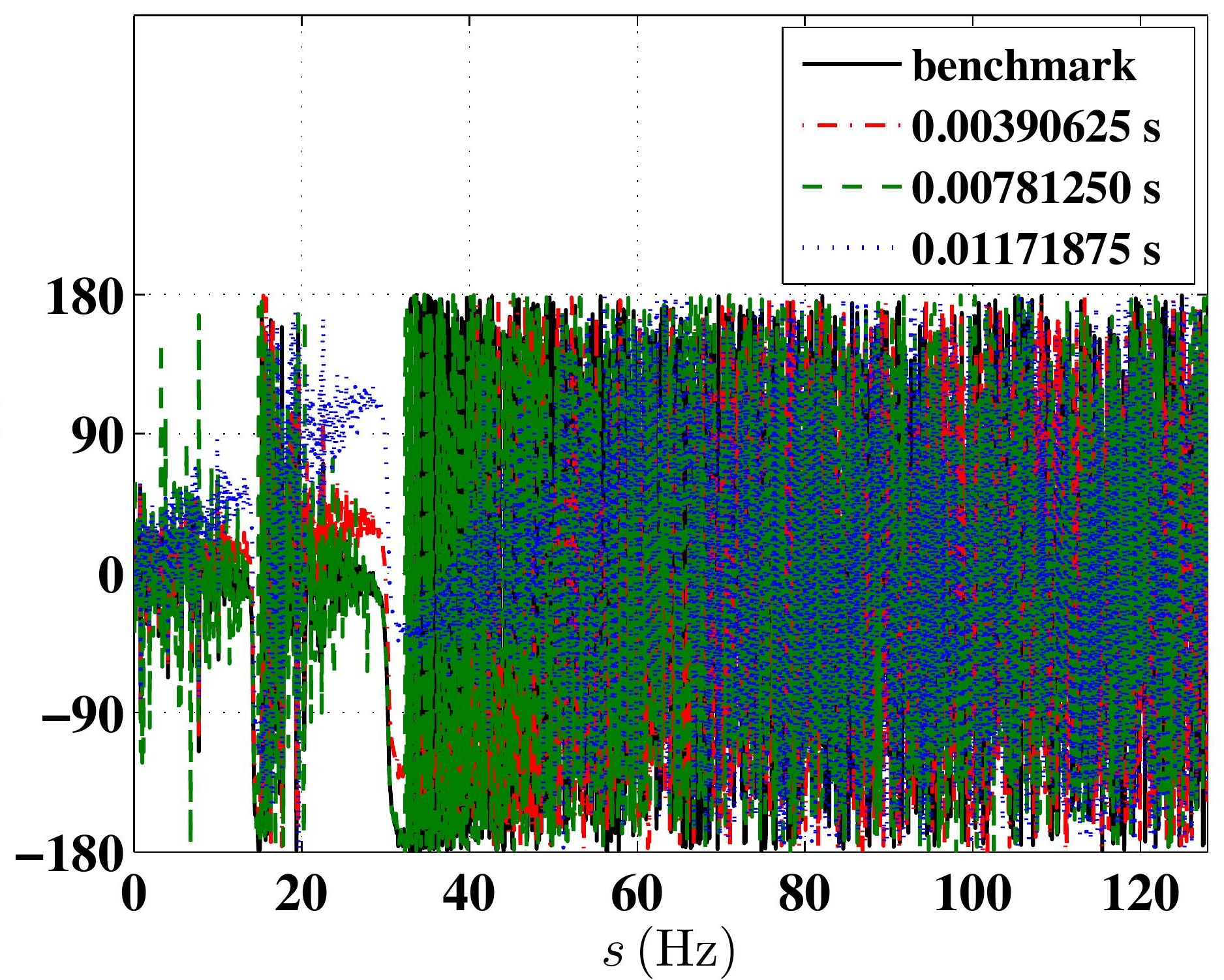




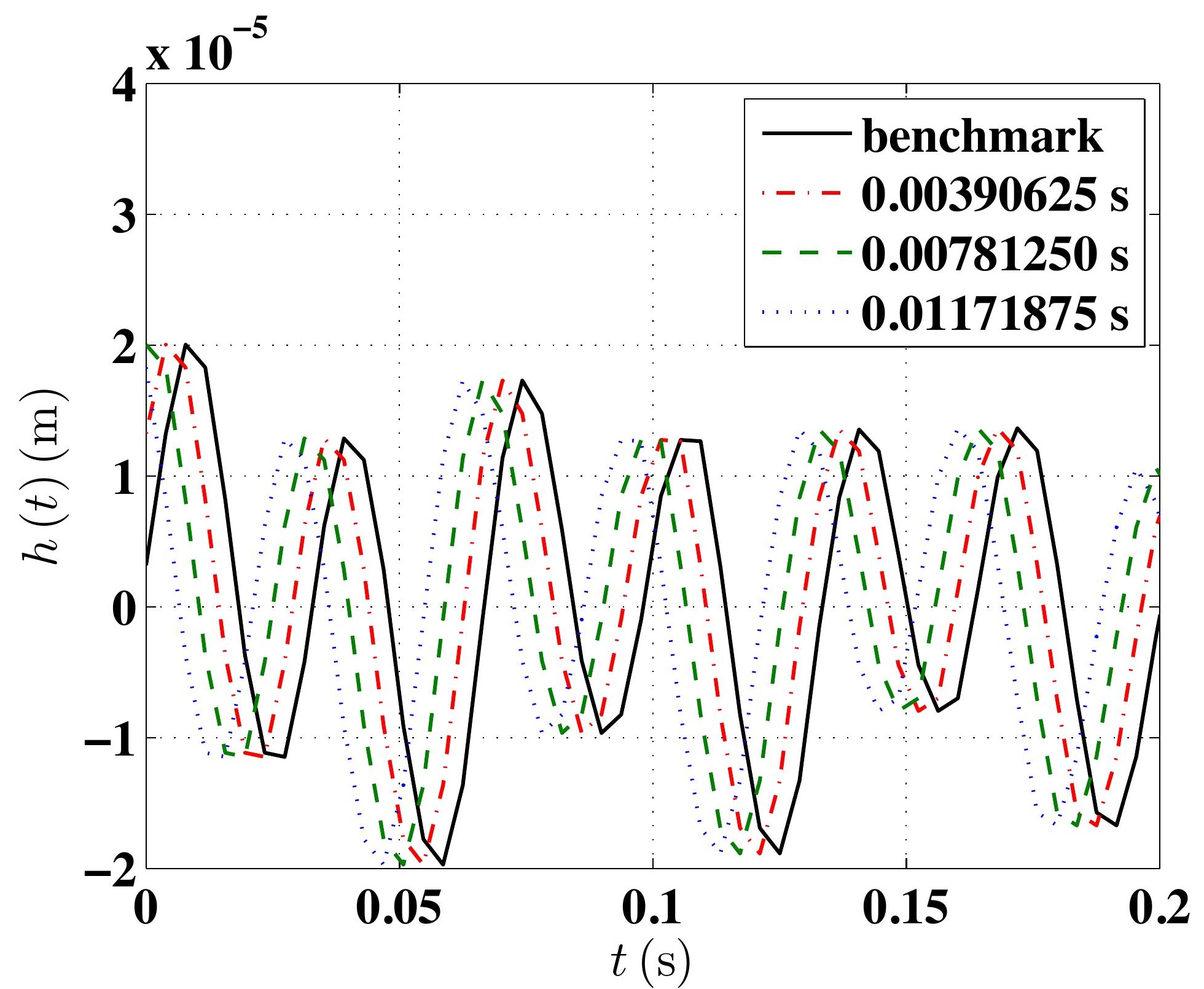


Figure $6 b$

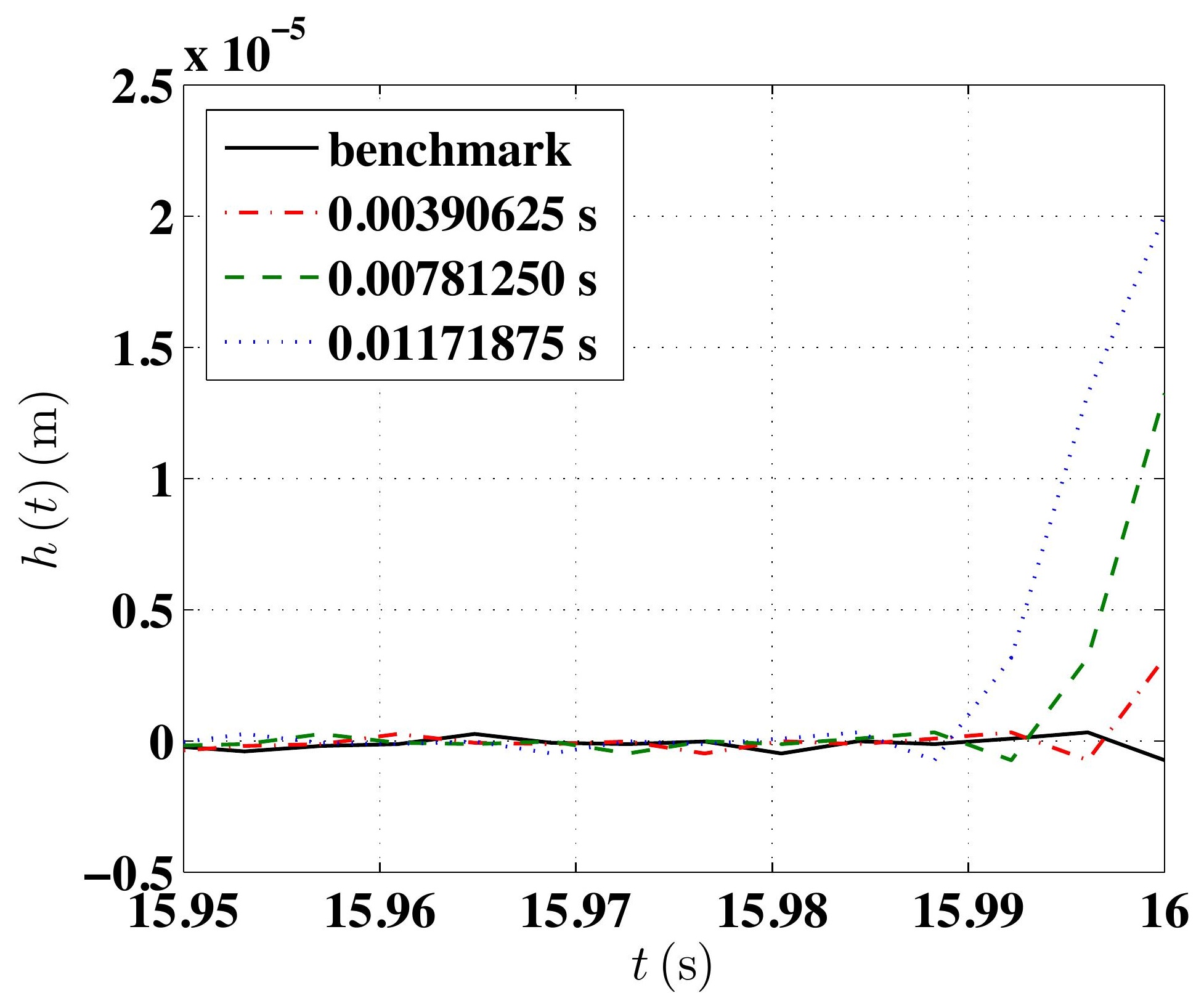




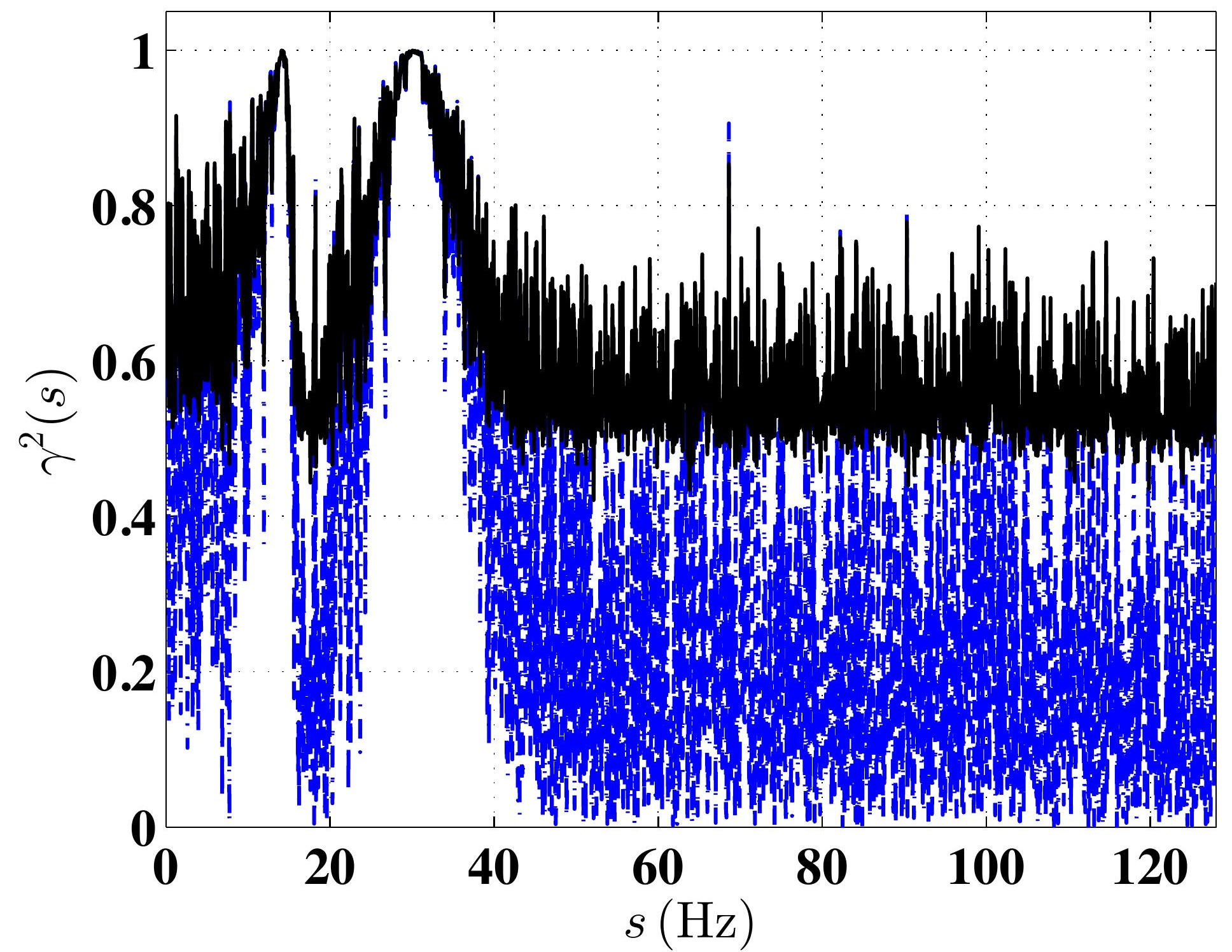




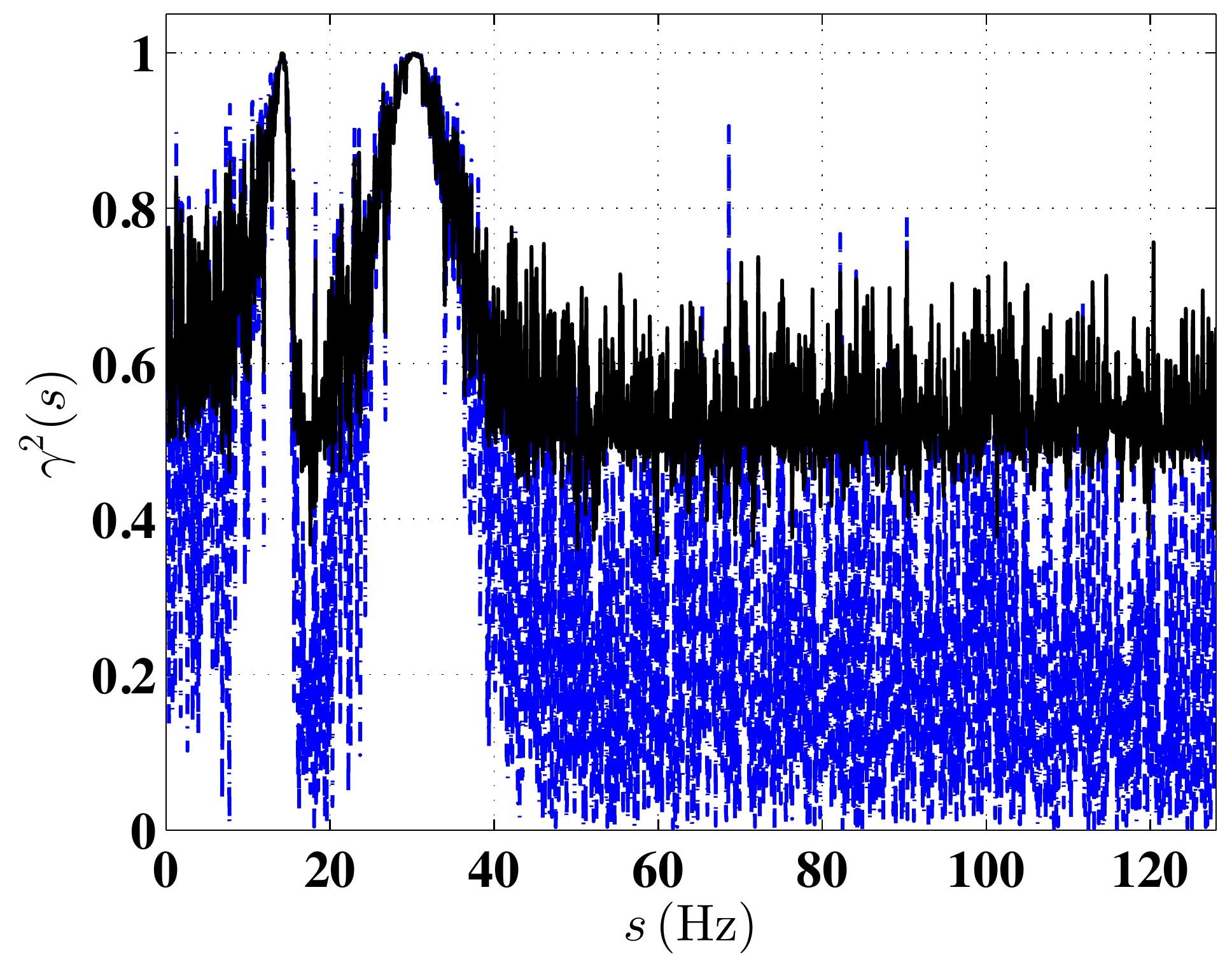




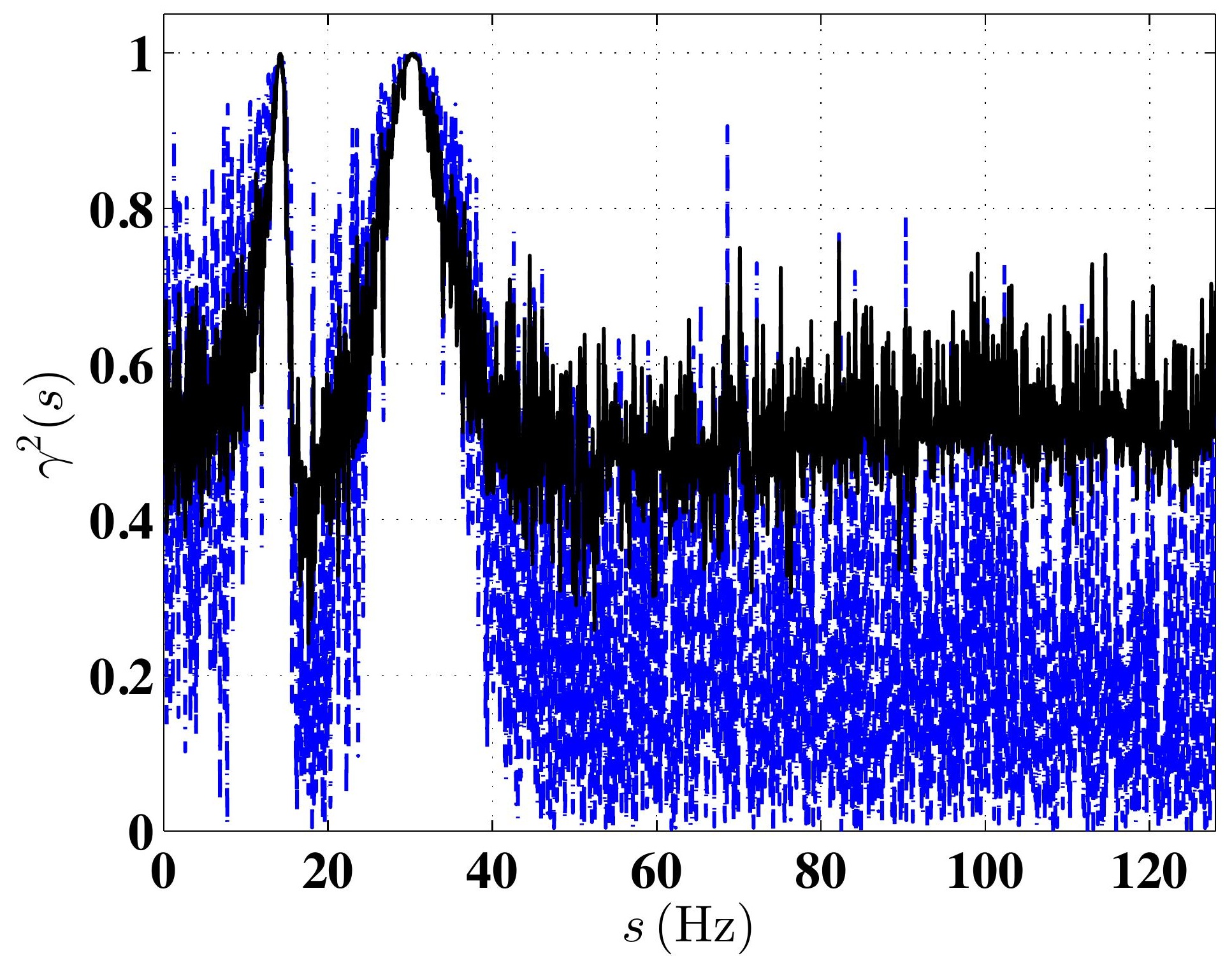




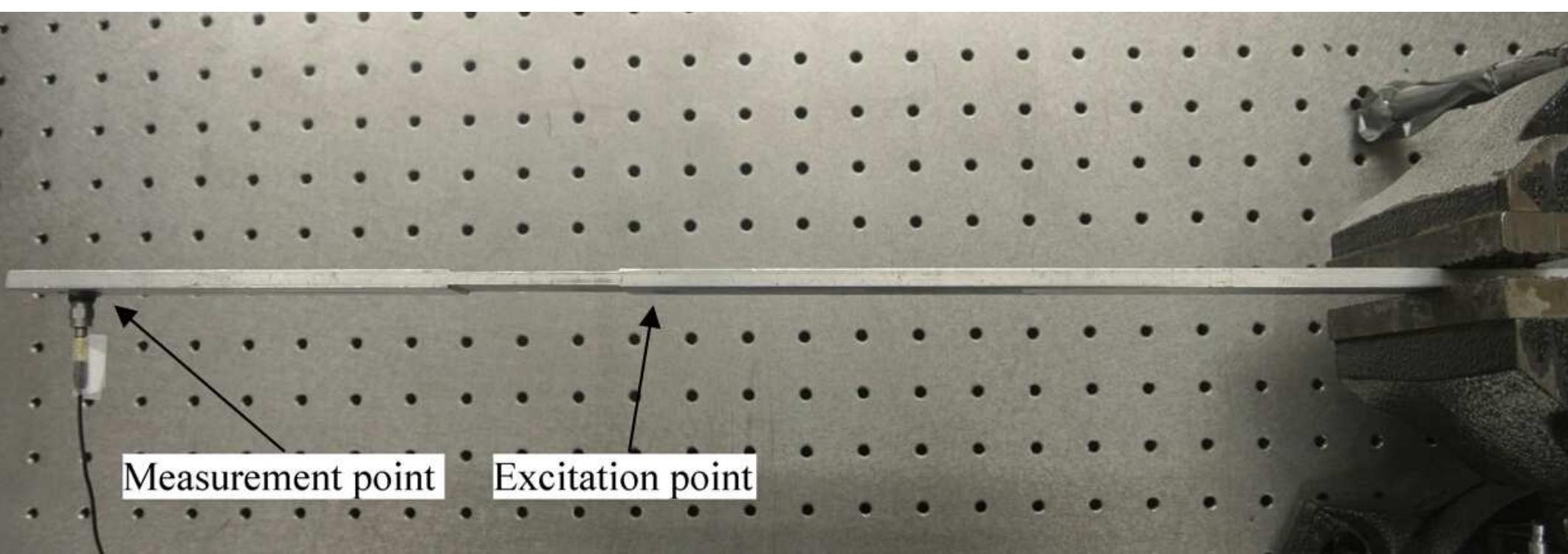

\section{Figure 8}

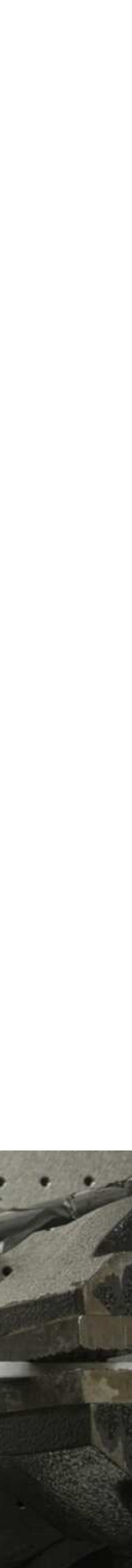


Figure 9a

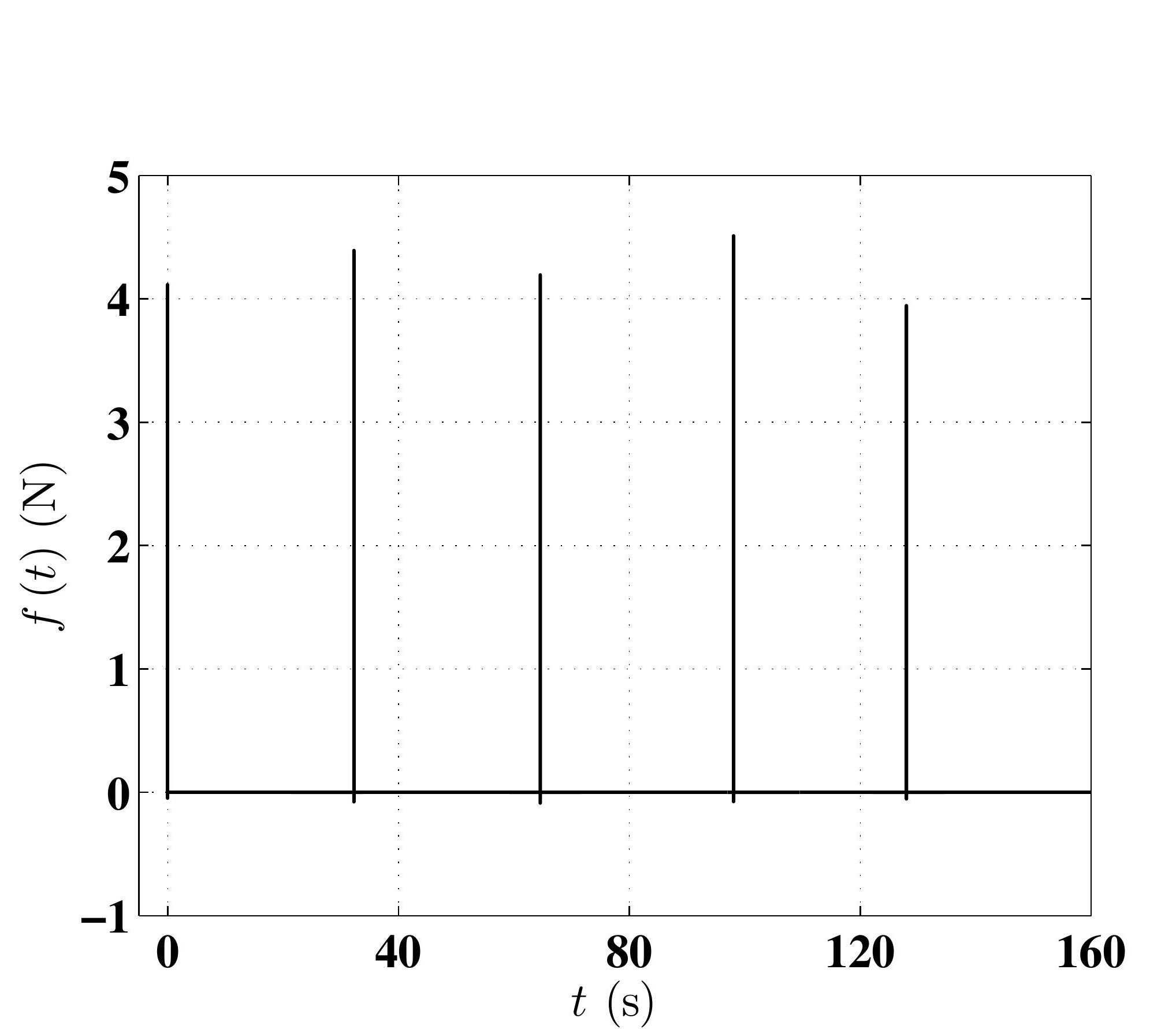

\section{Figure 9a}


Figure 9b

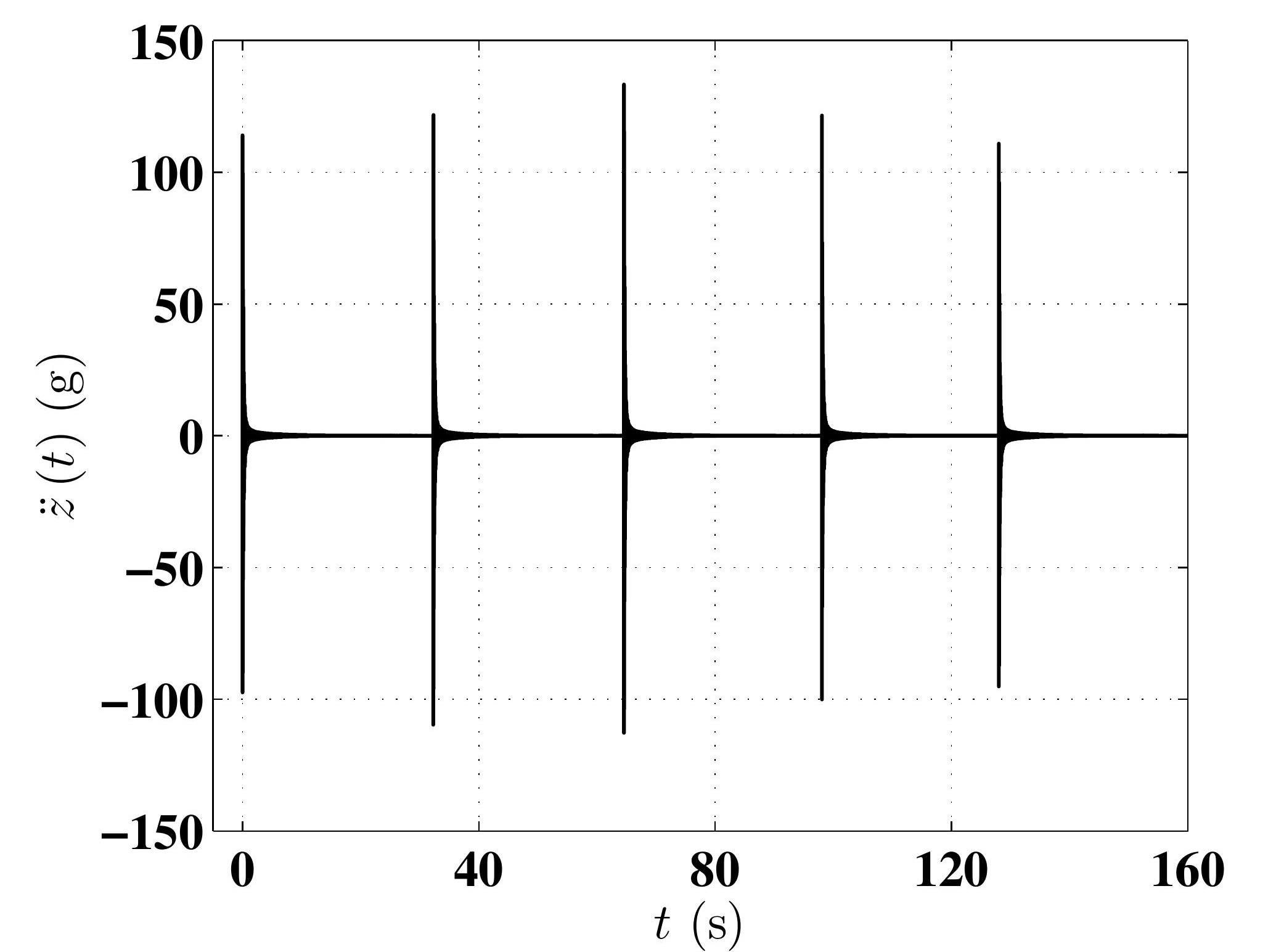


Figure $10 a$

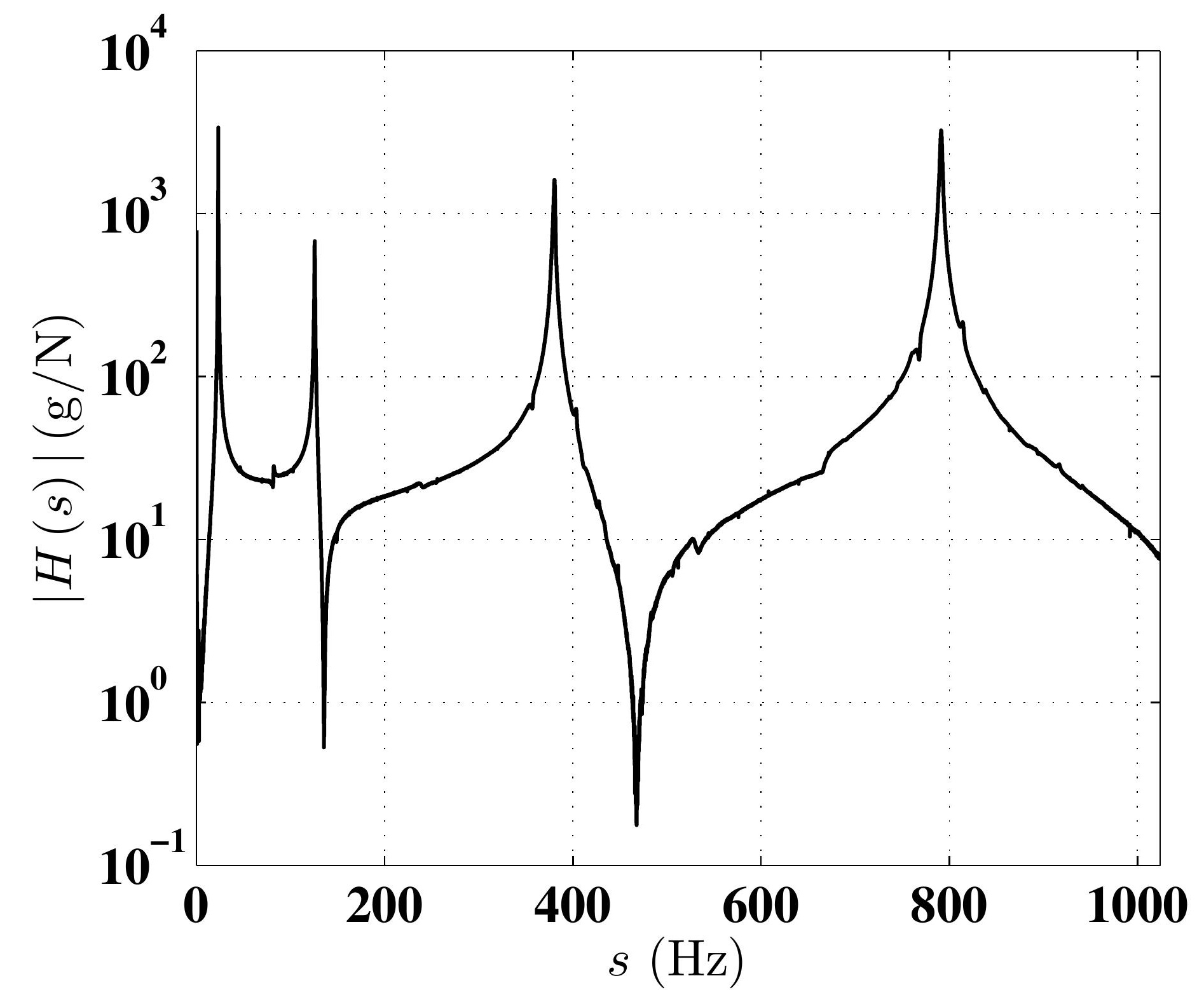


Figure 10b

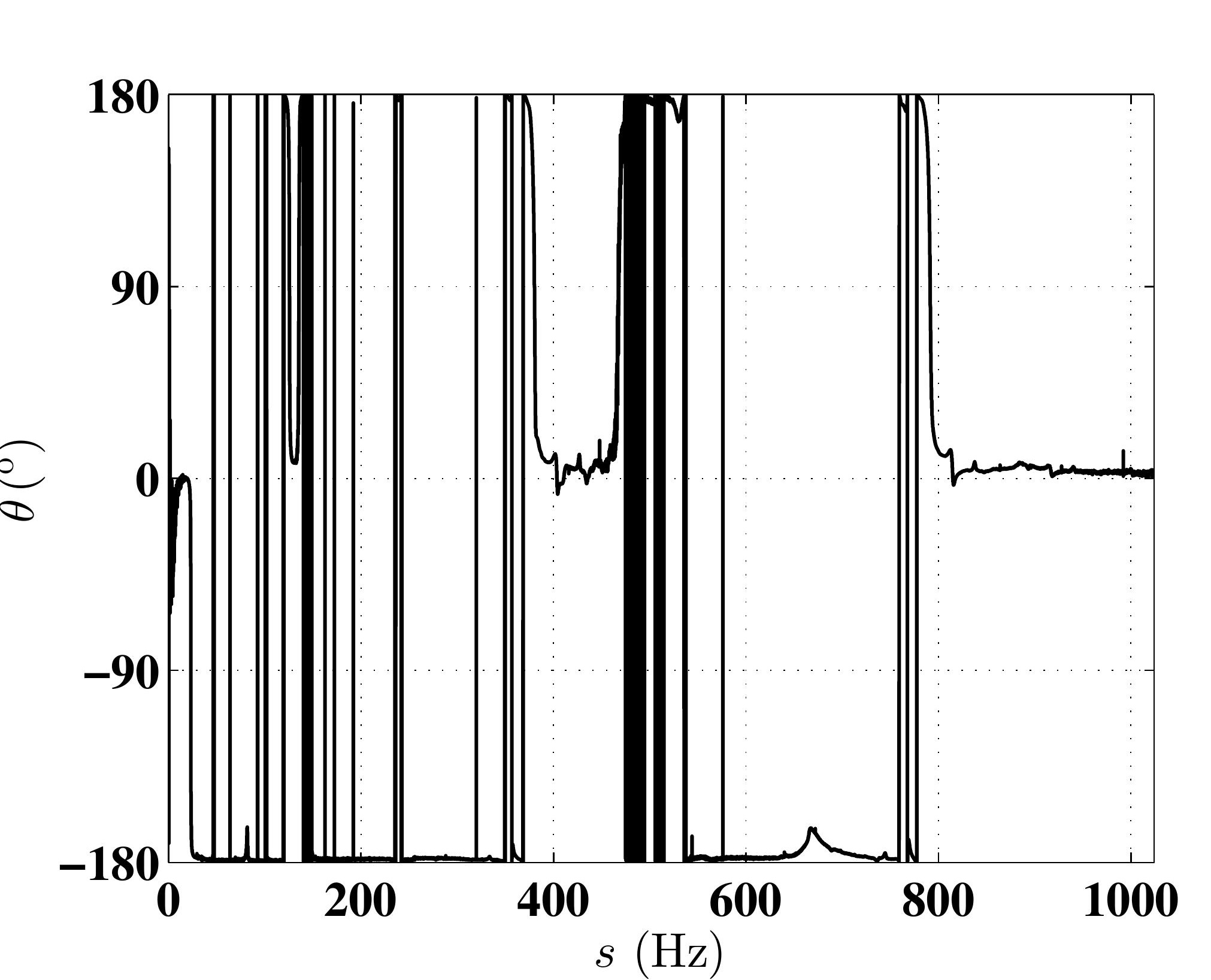

\section{Fure 10b}

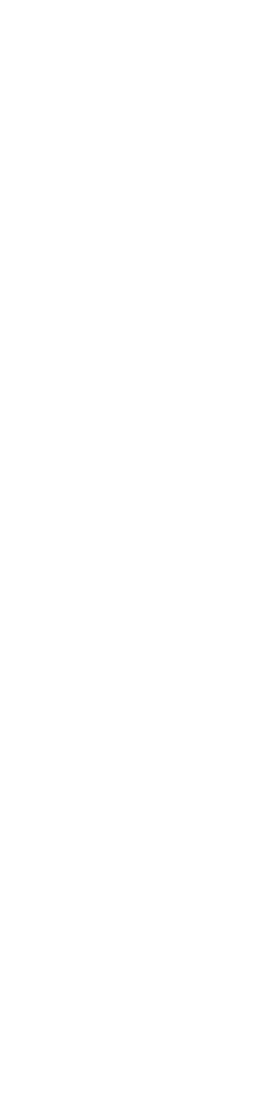

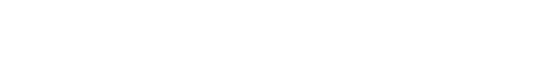

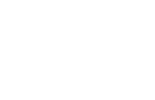

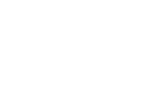

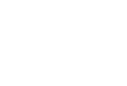

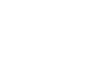


Figure $10 \mathrm{e}$
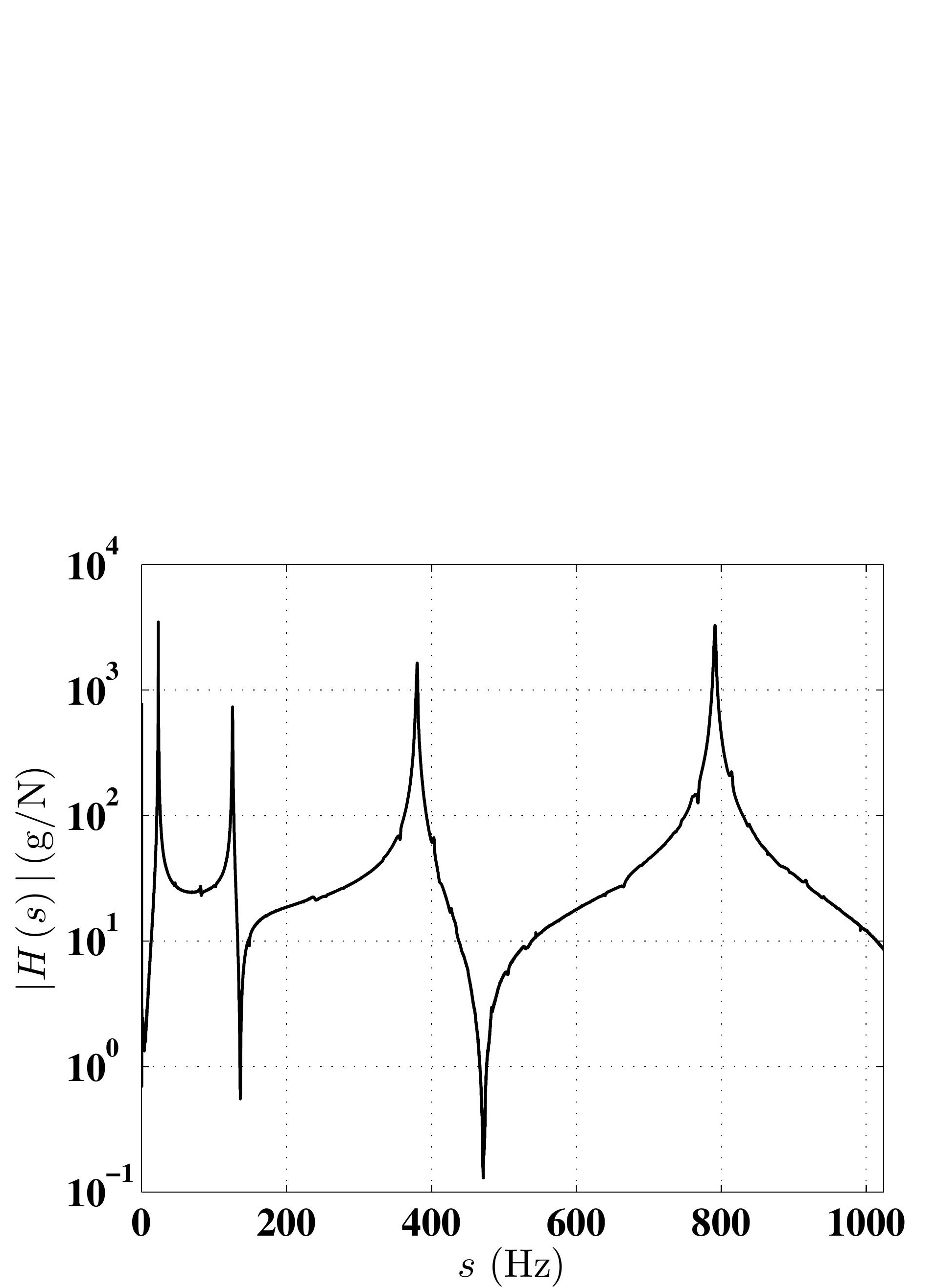

. 
Figure 11a

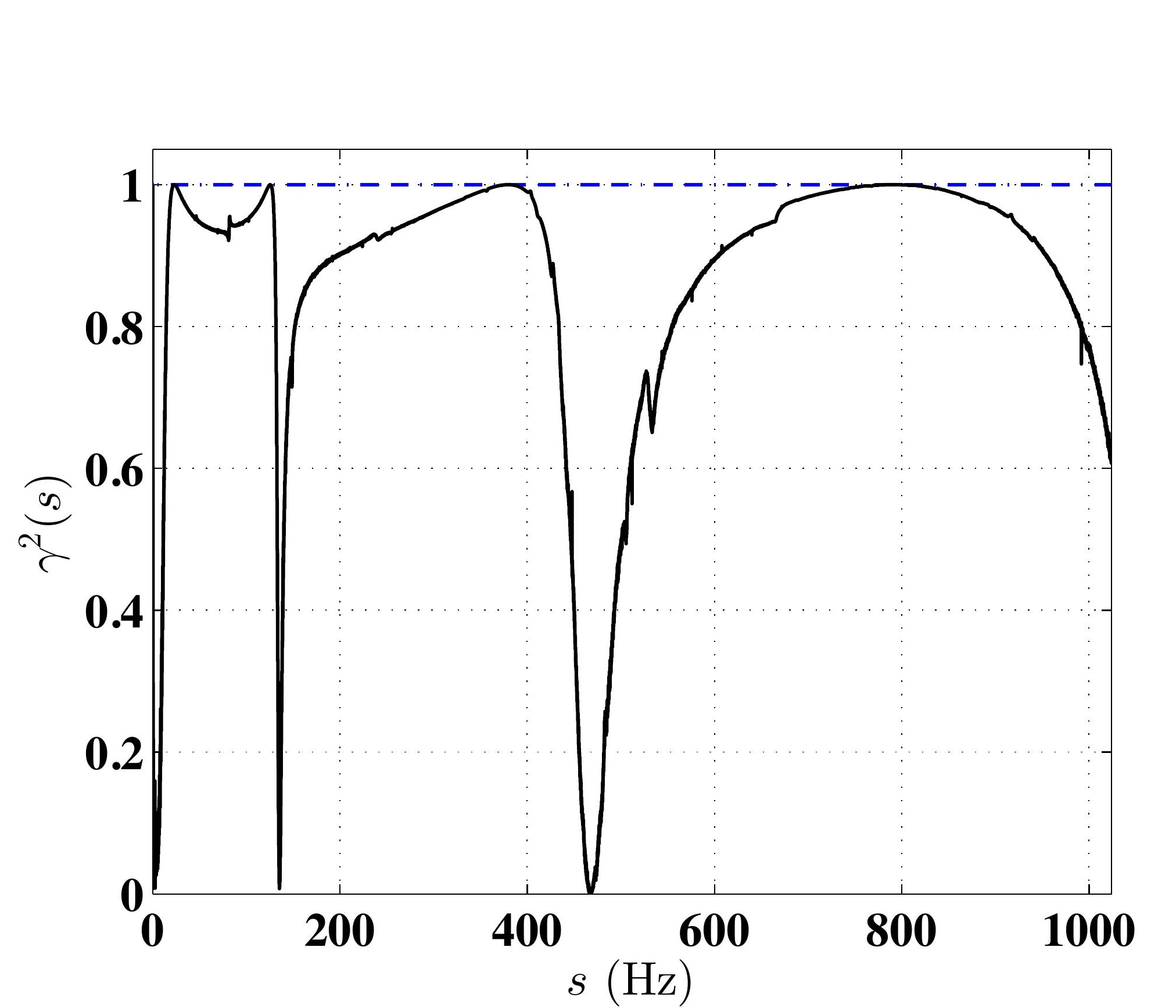

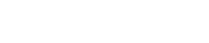




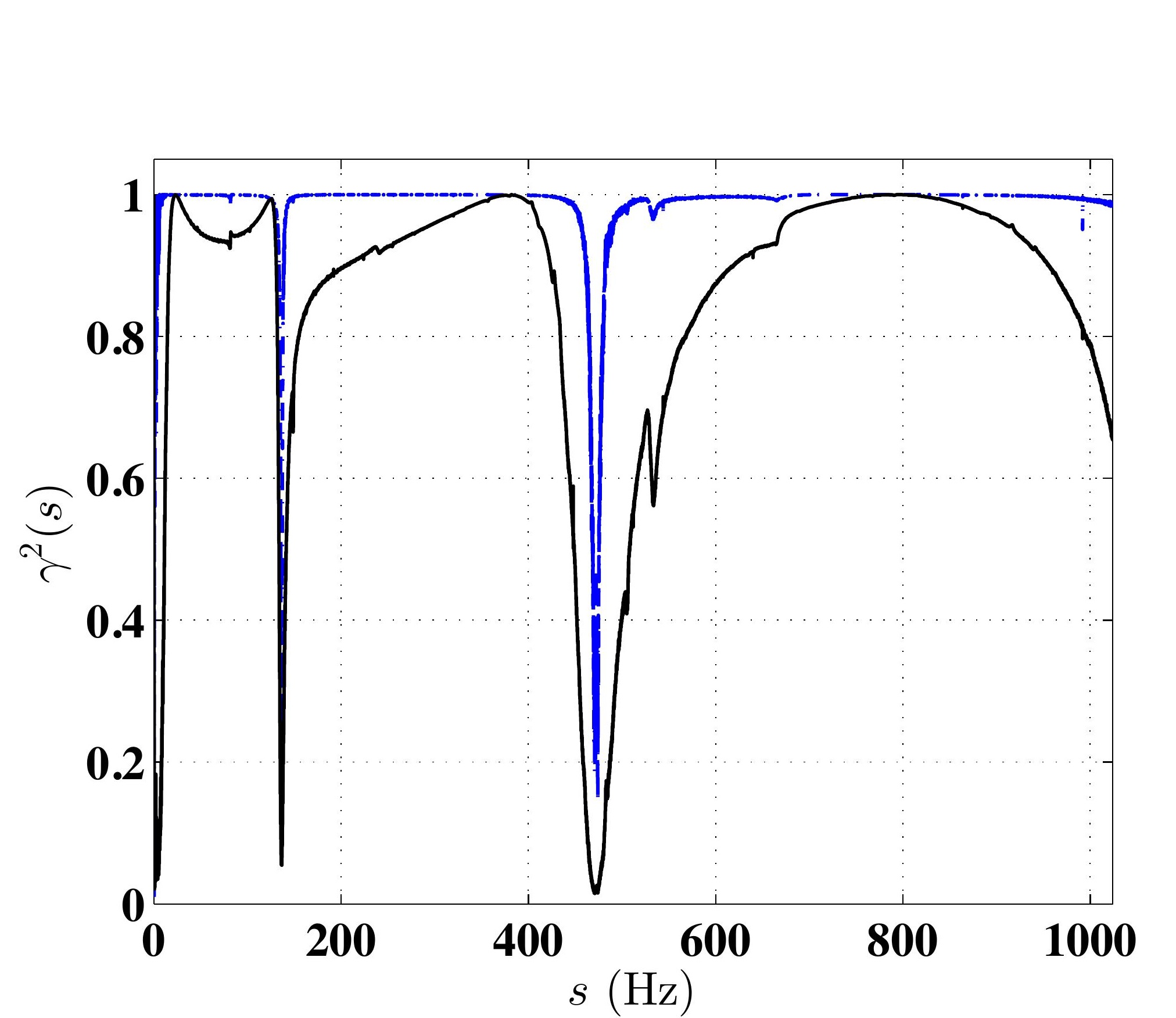

Figure 11b

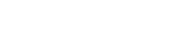




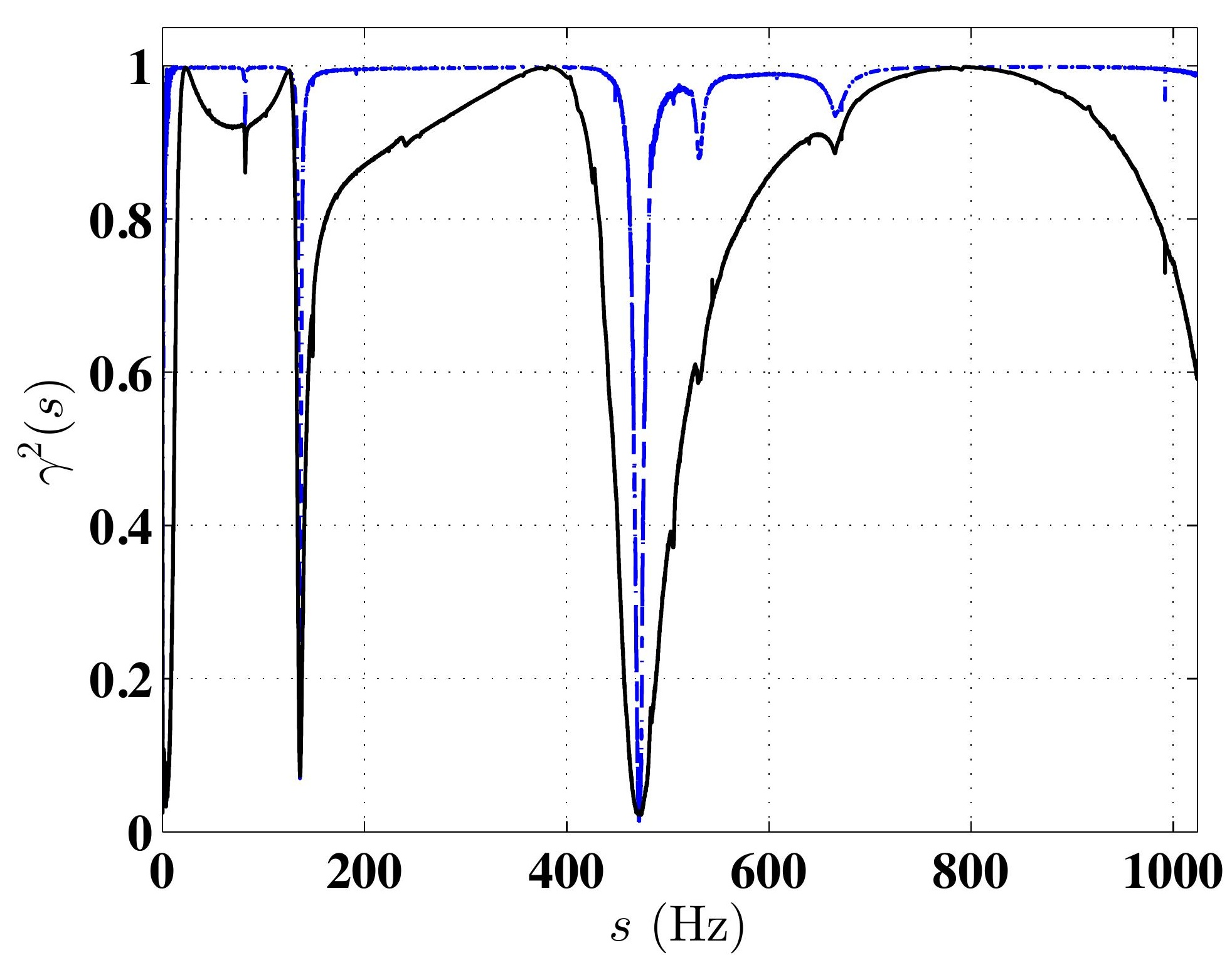

Figure 11c 
Figure 12a

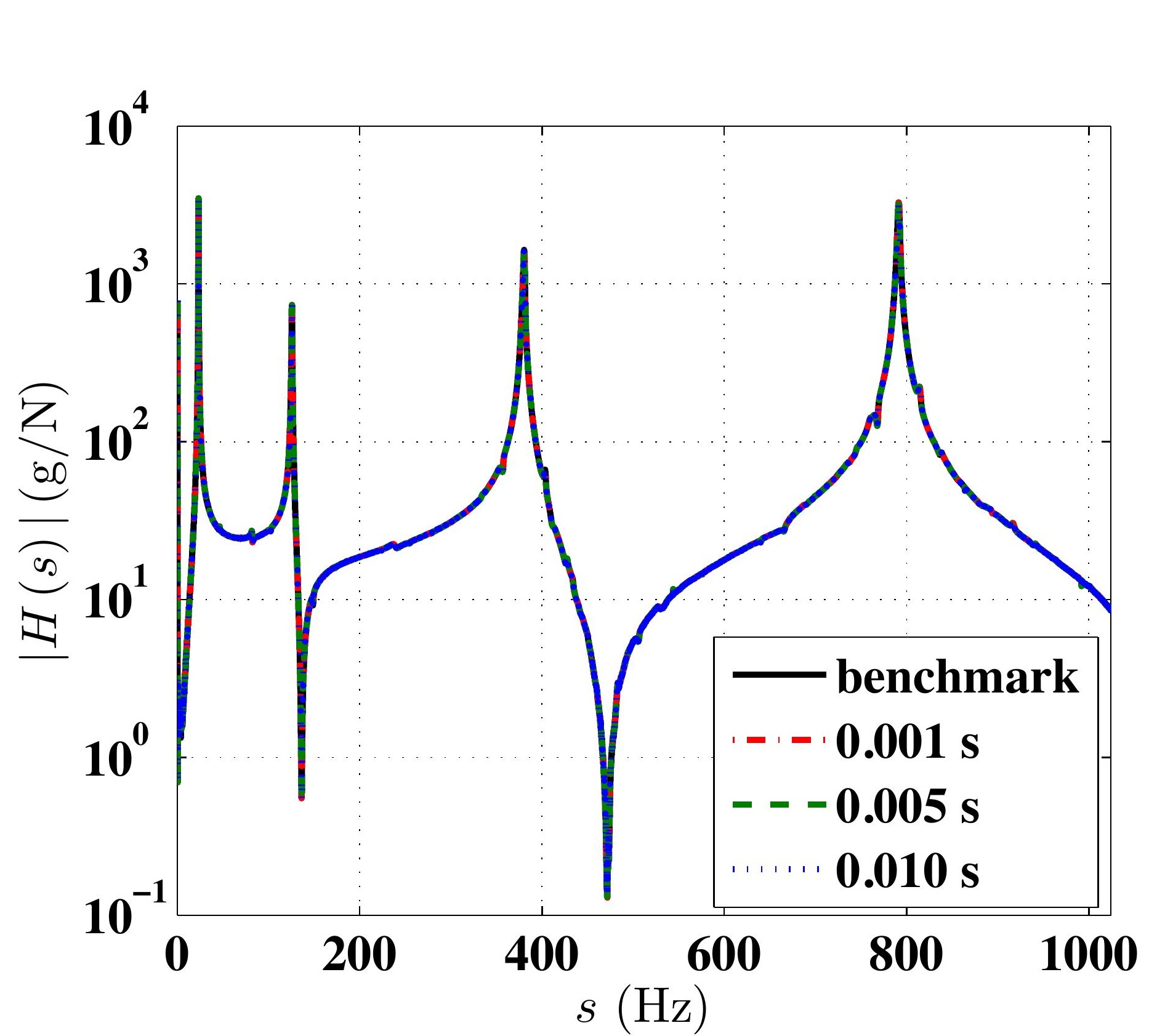




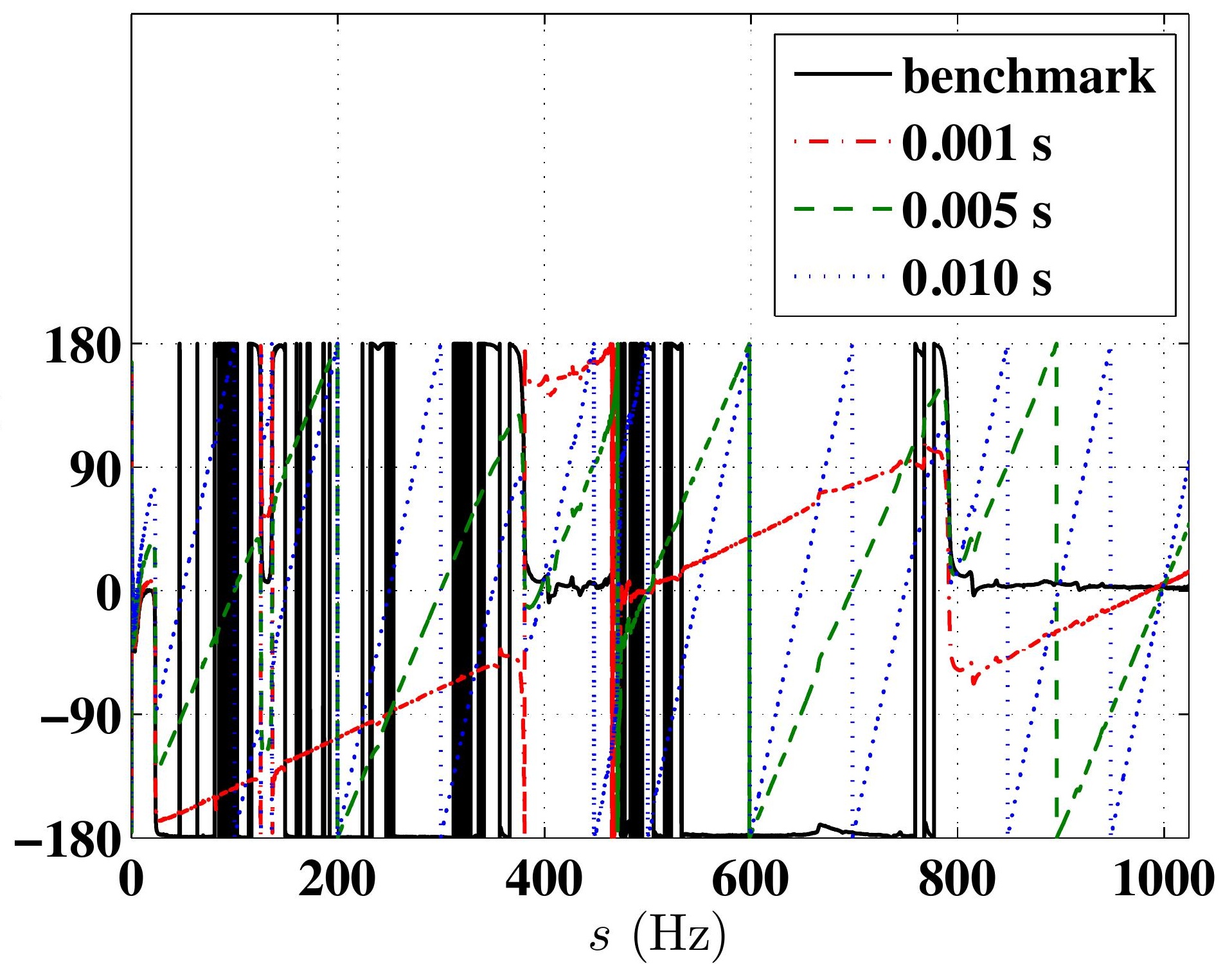




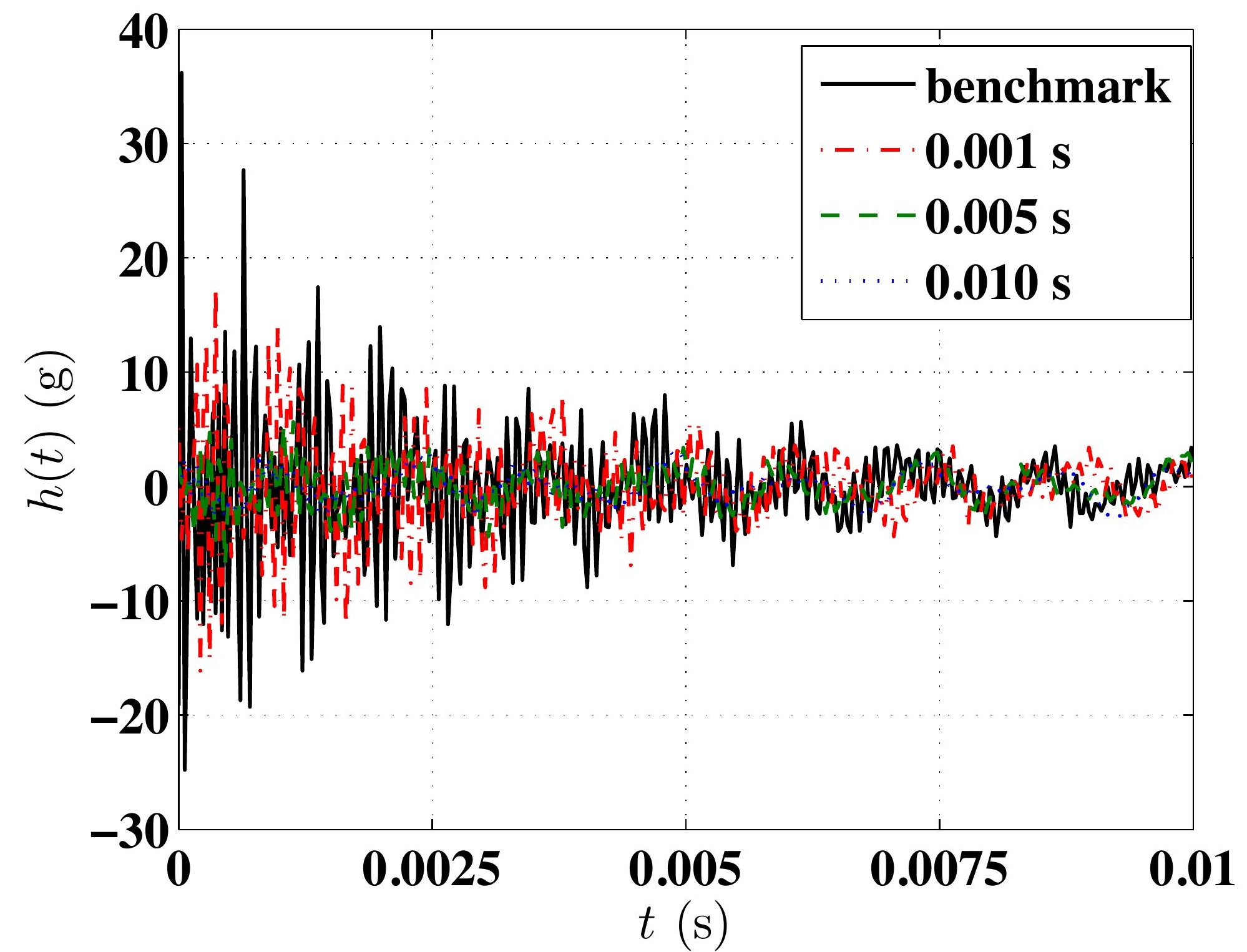




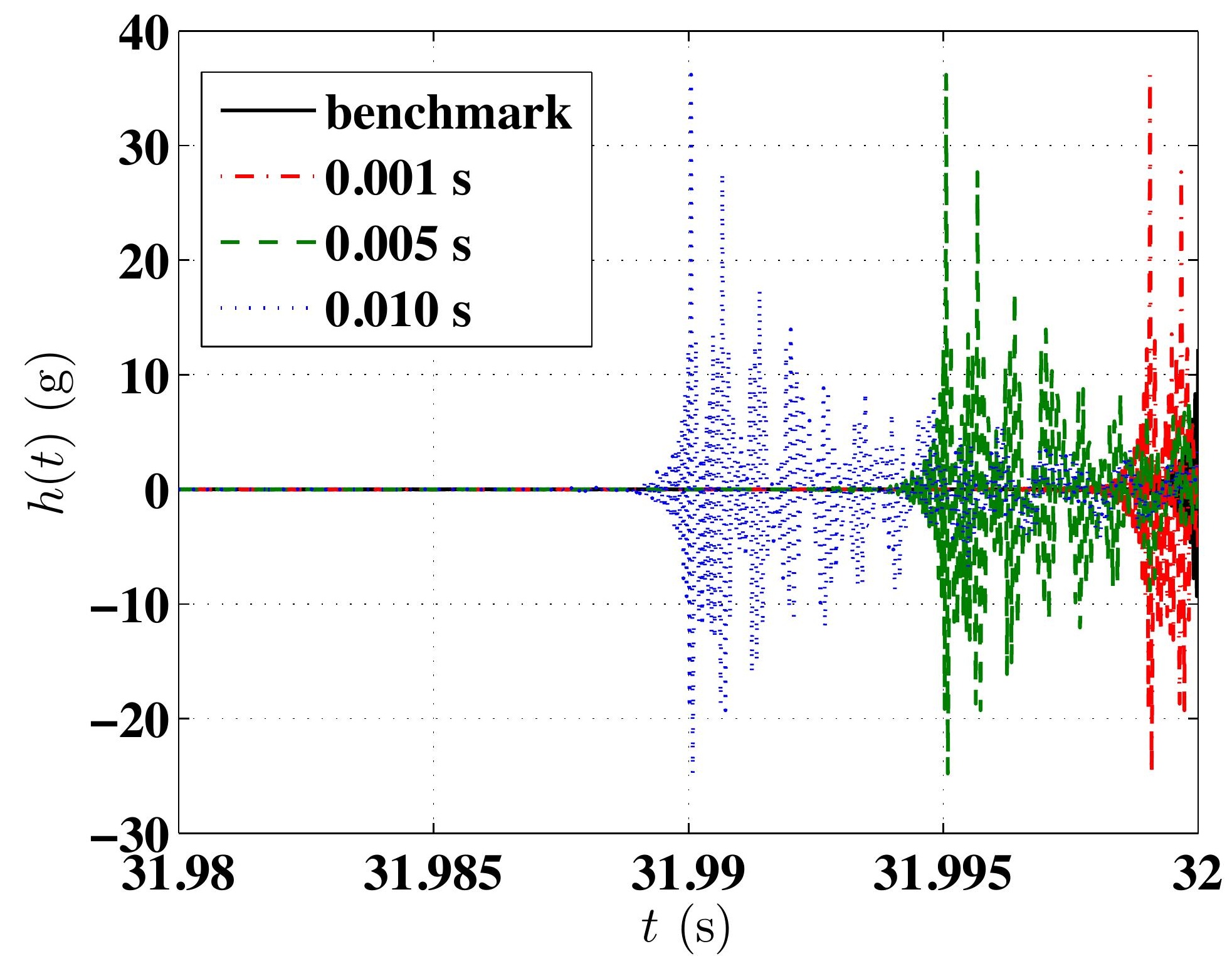




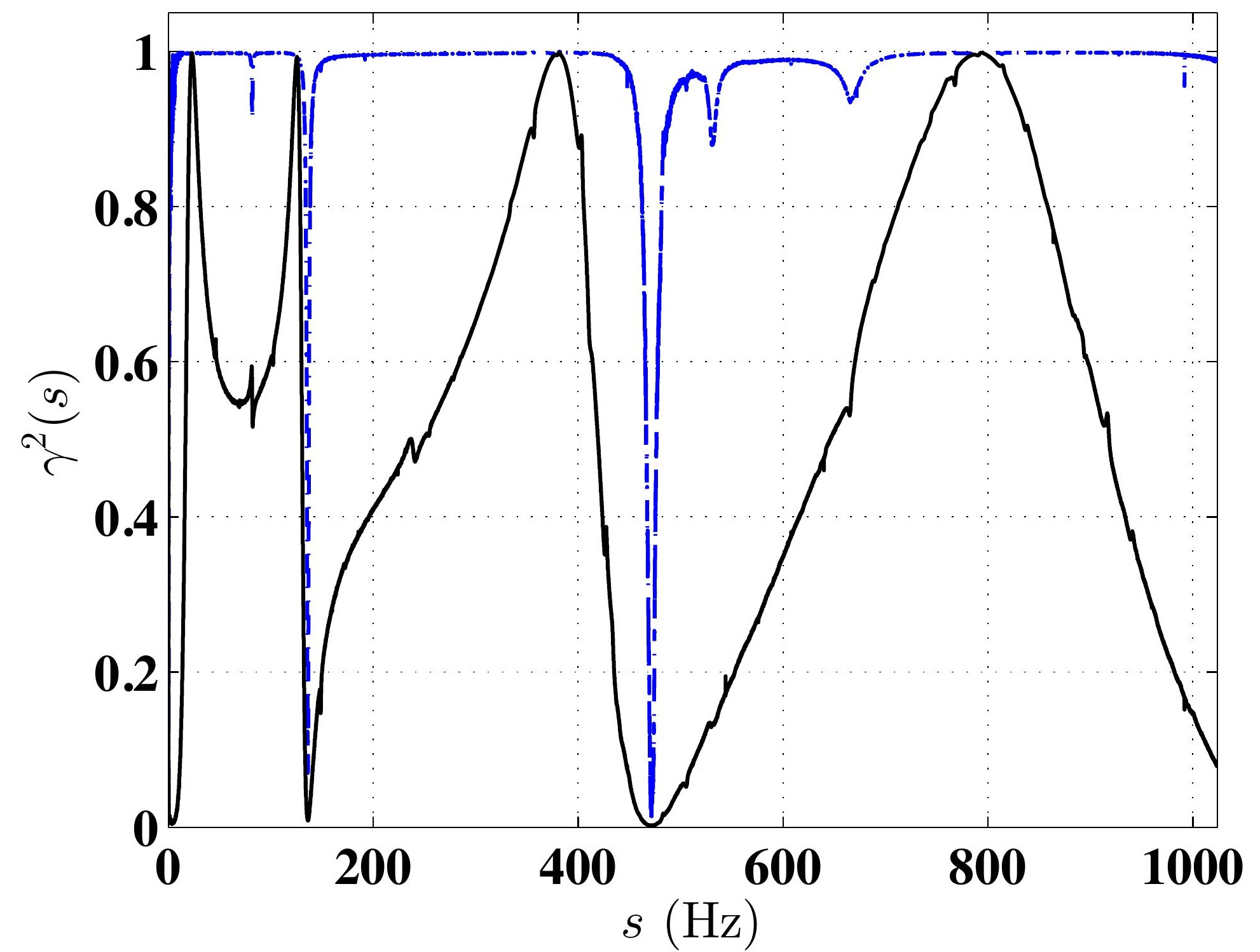




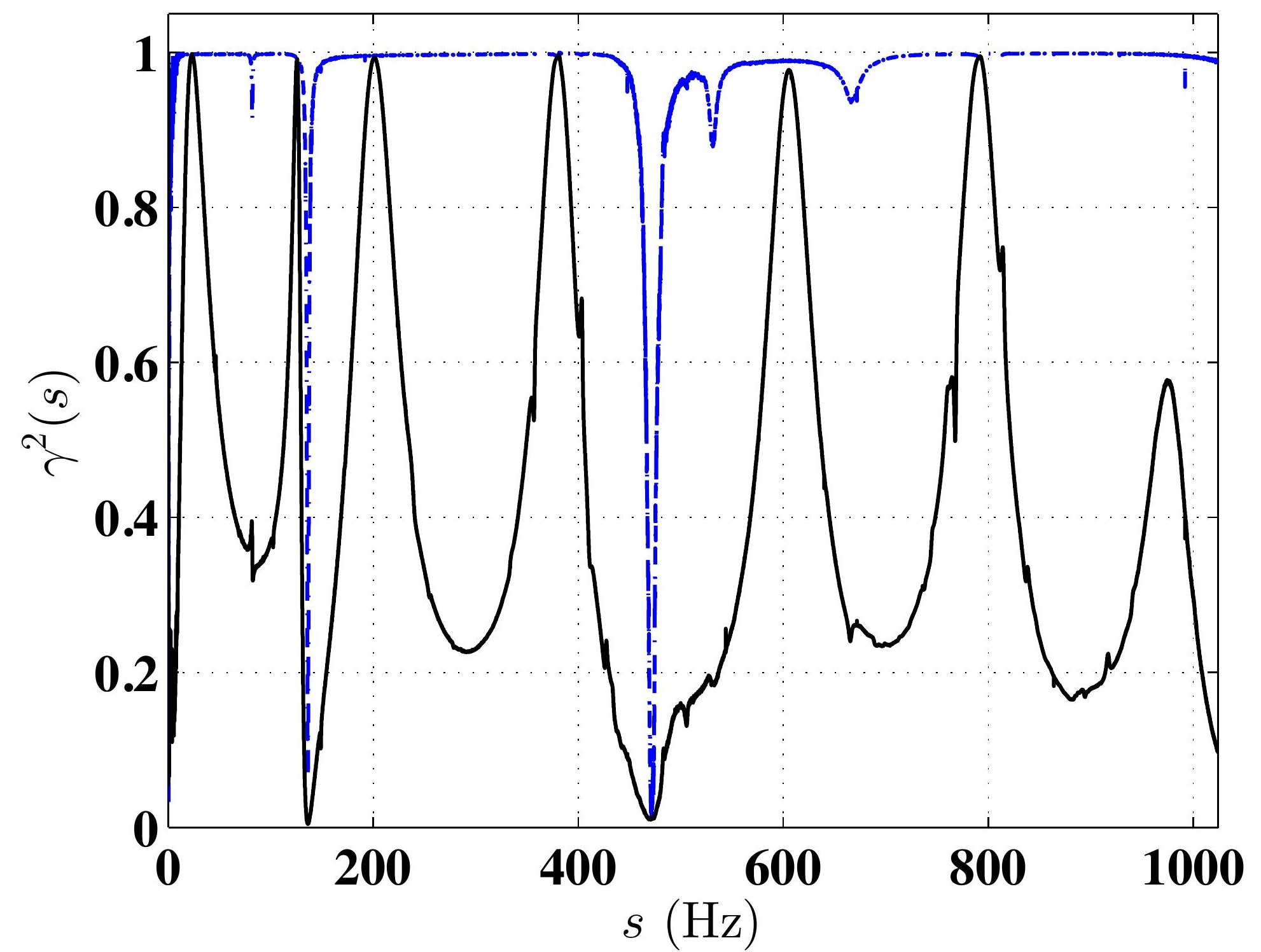




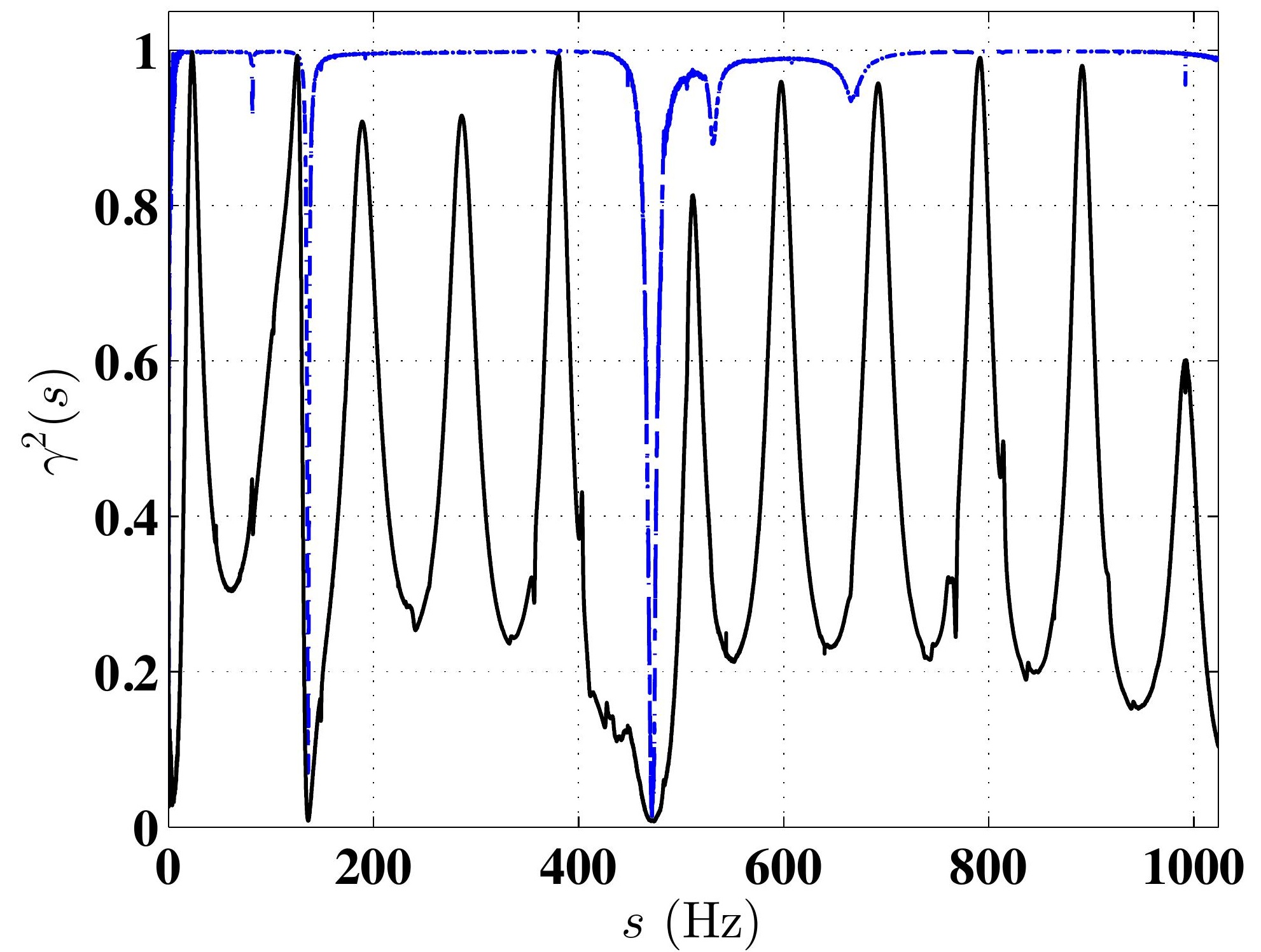




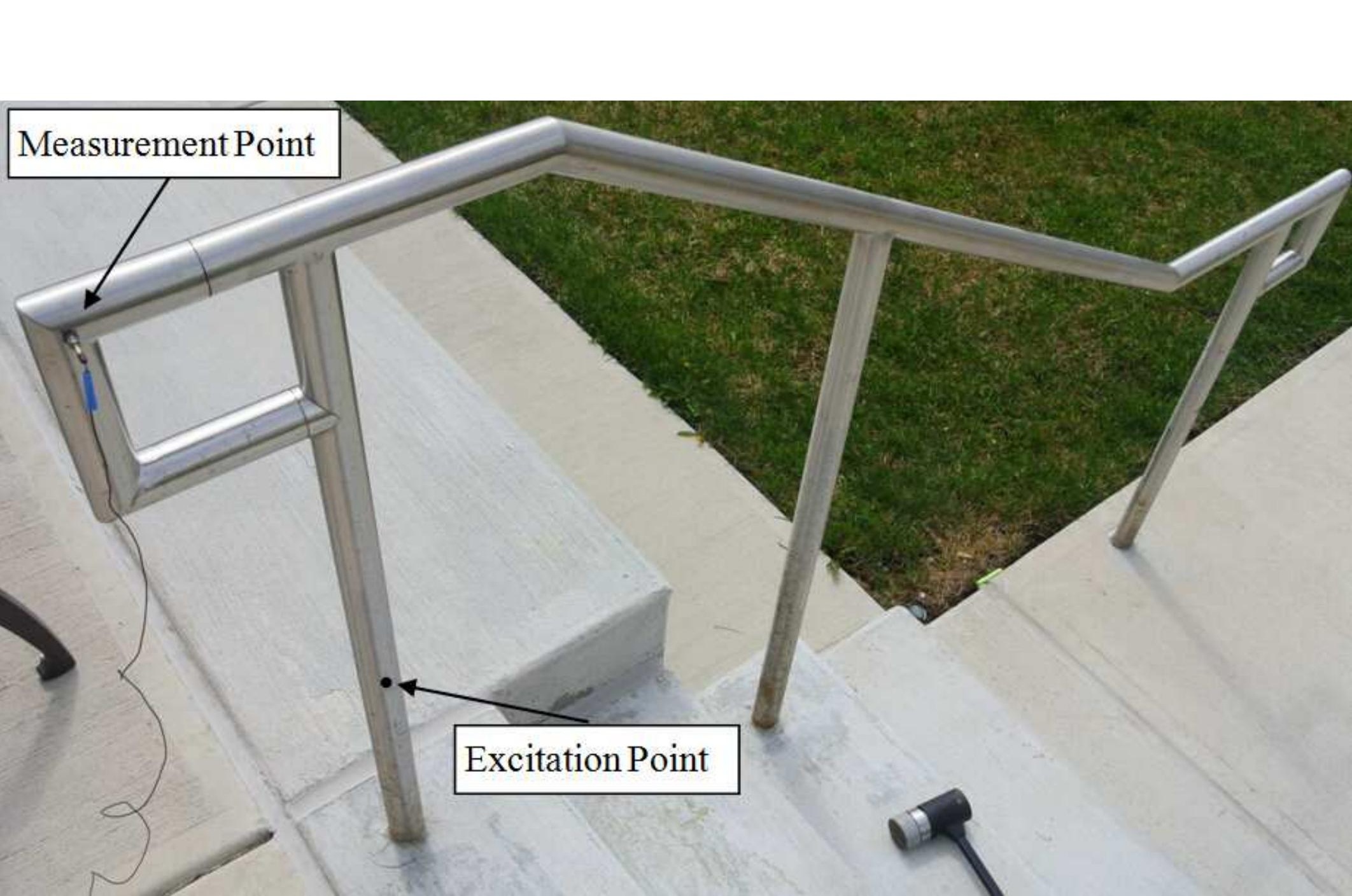

Figure 15 


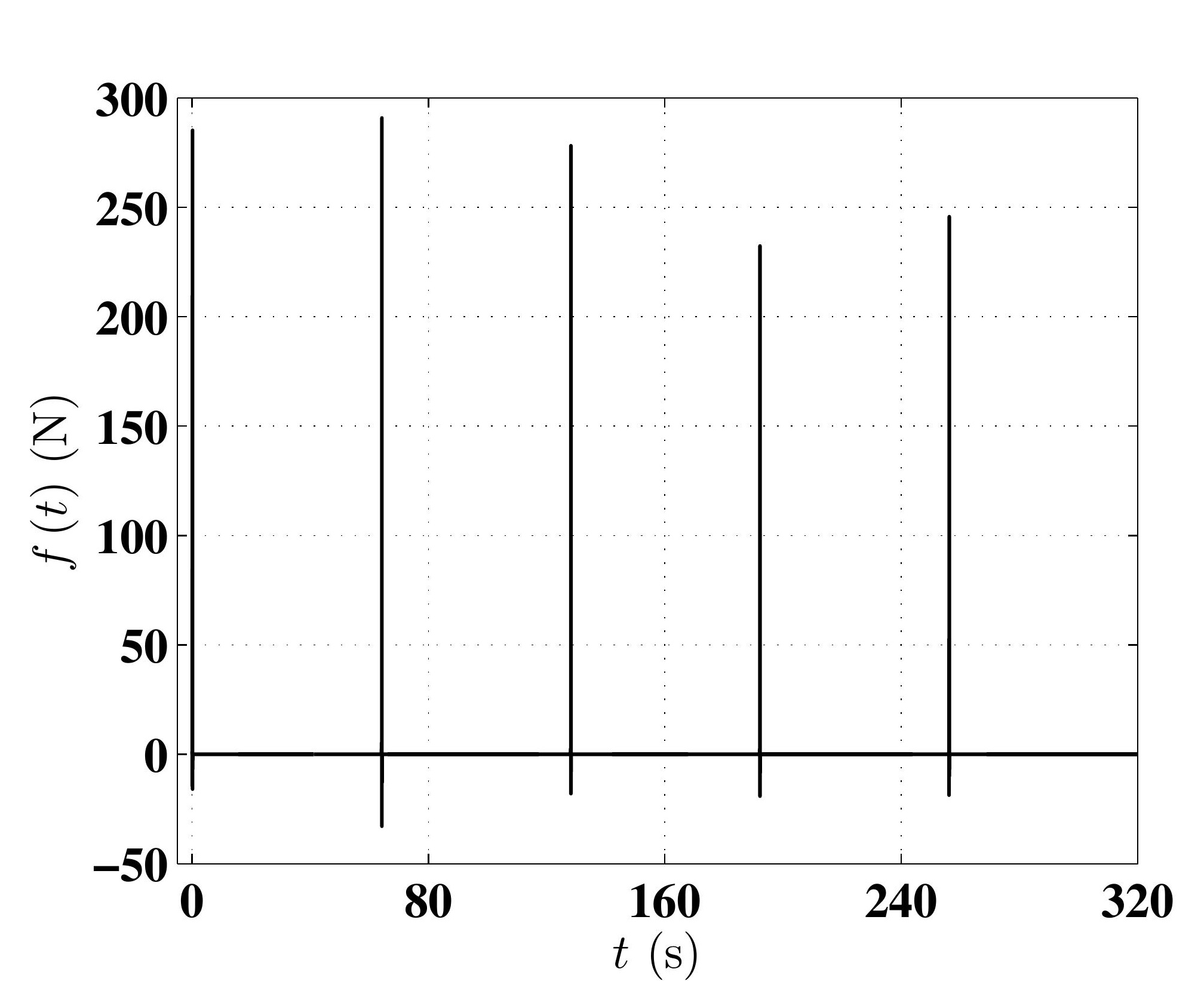

Figure 16a

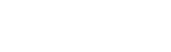

.




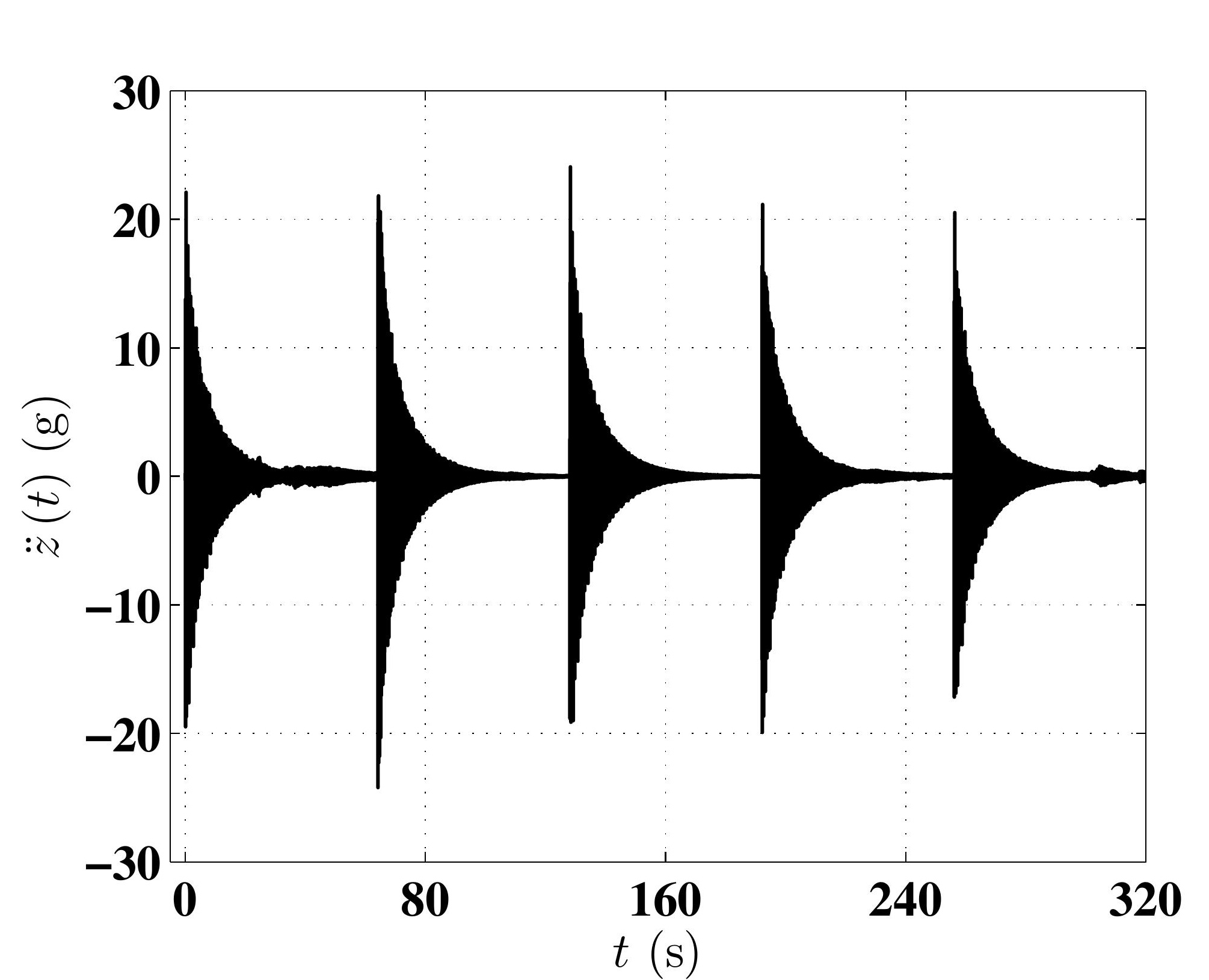

Figure 16b

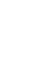

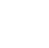

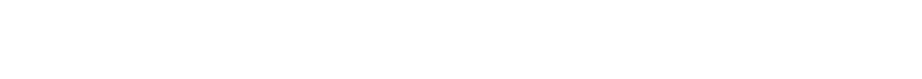

政

(

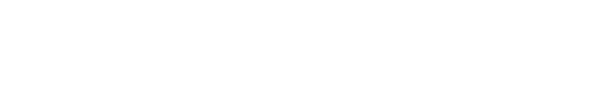

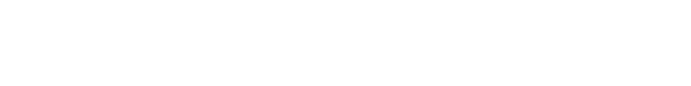

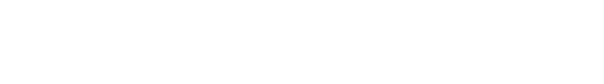

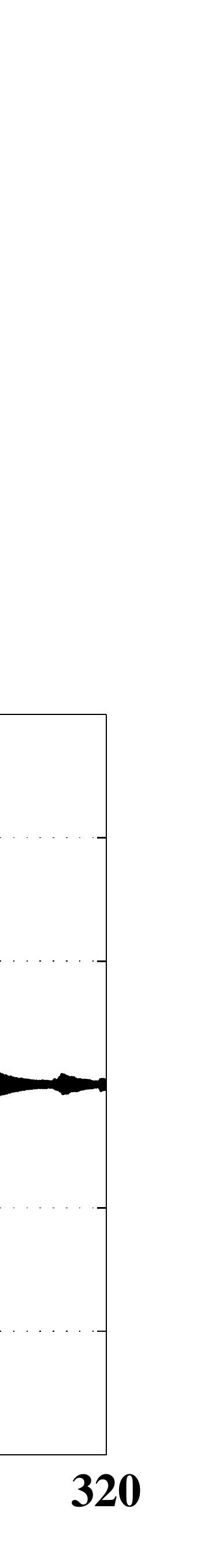




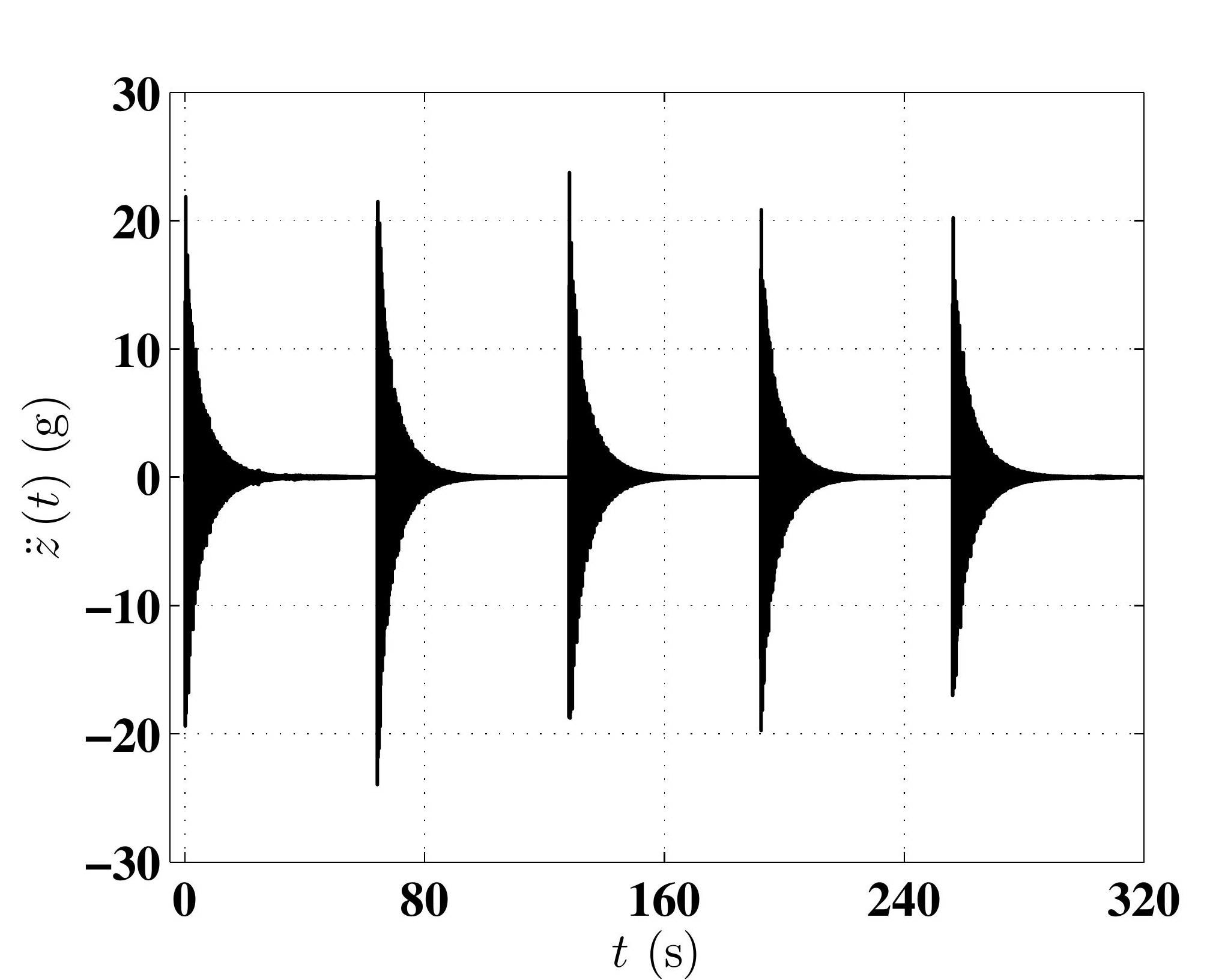

Figure 16c

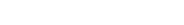

.
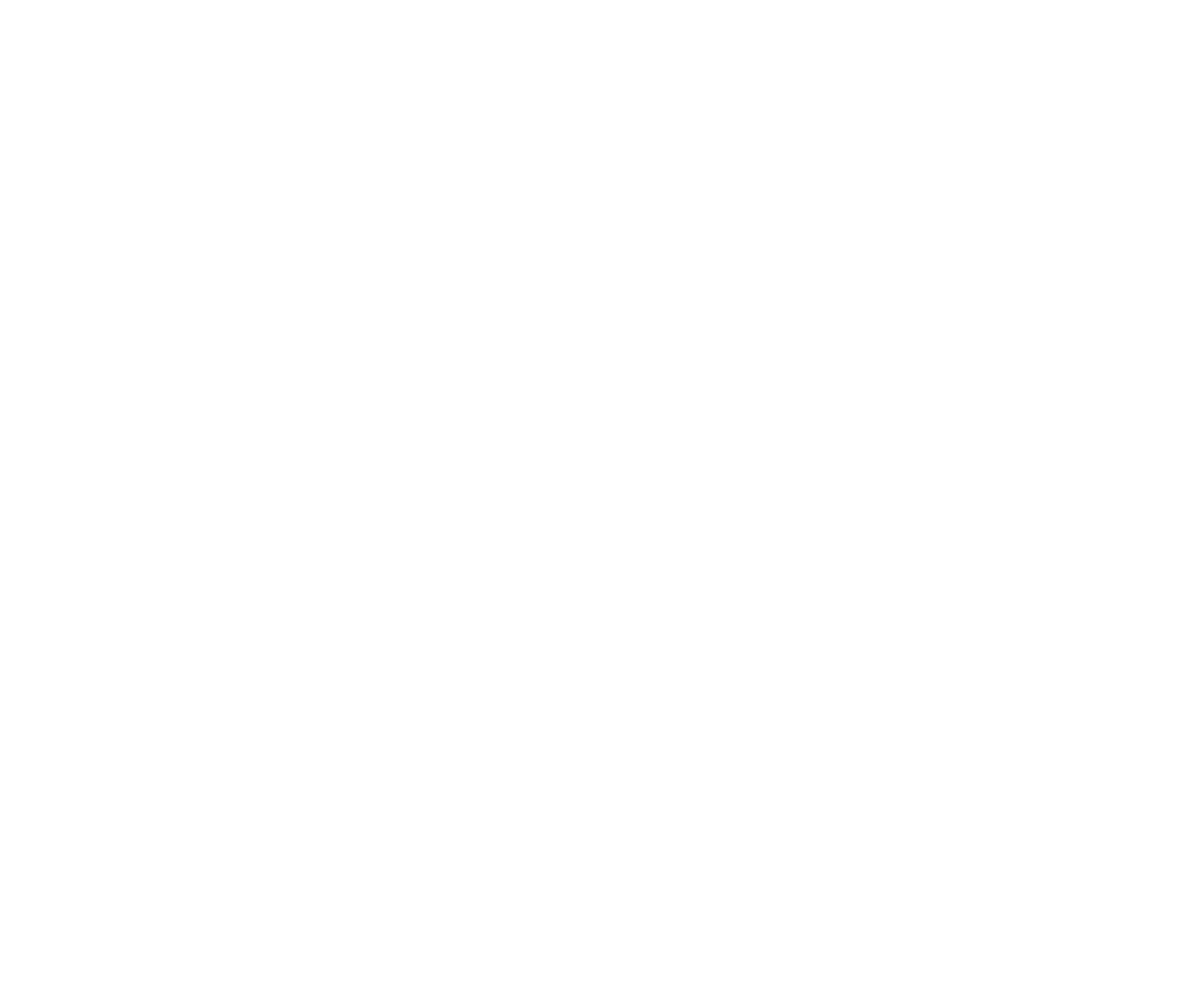


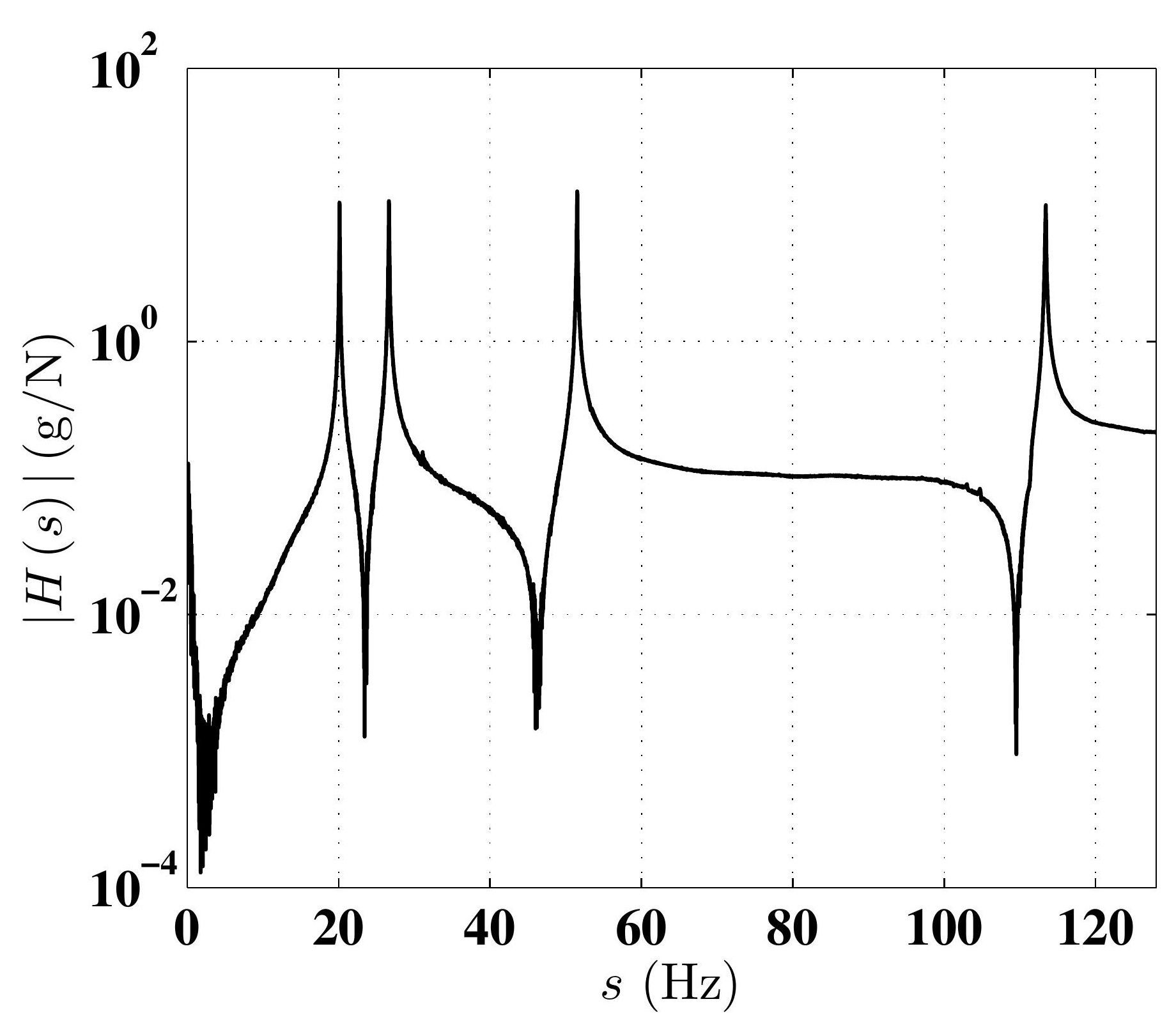

Figure 17a 
Figure 17b

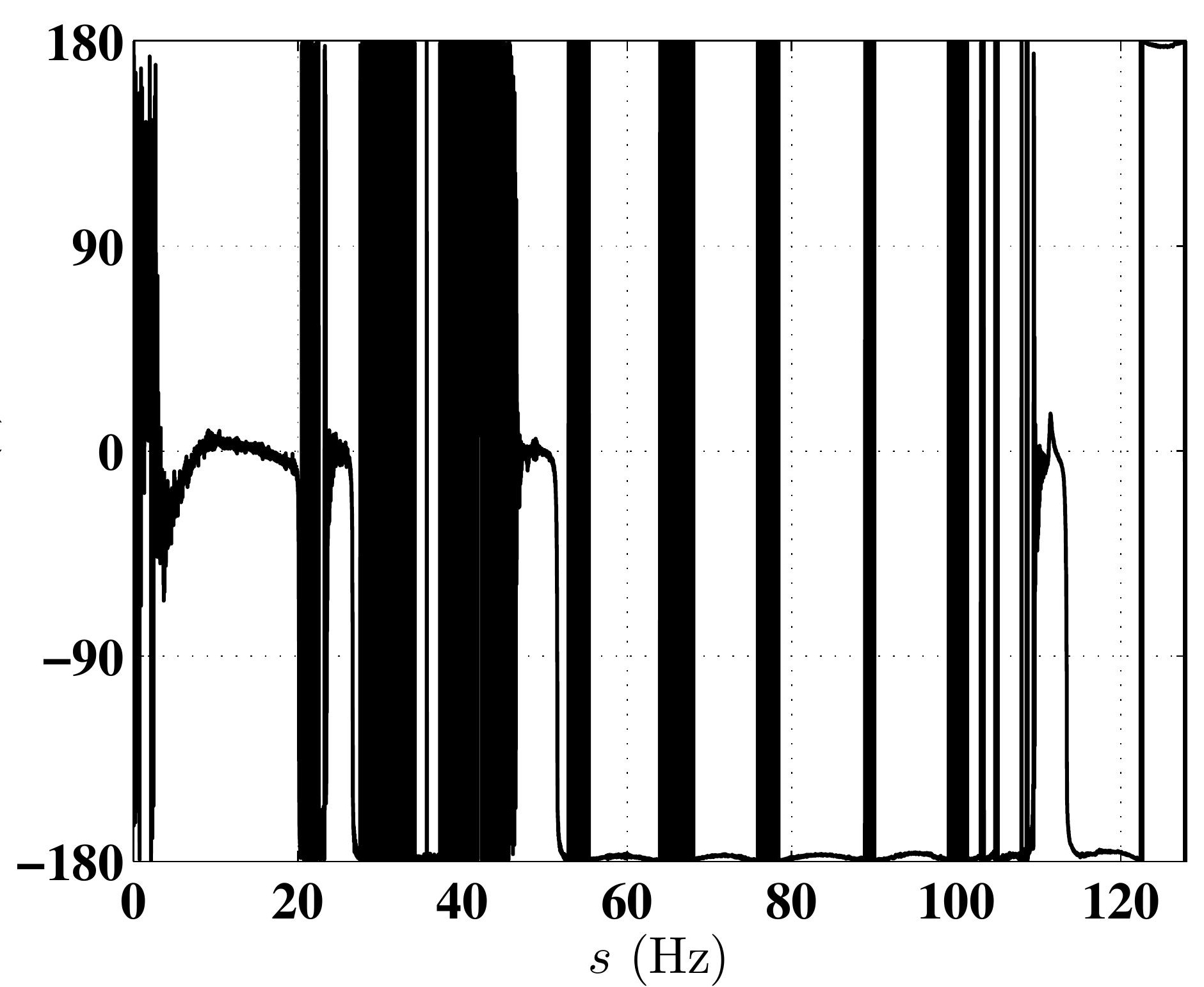




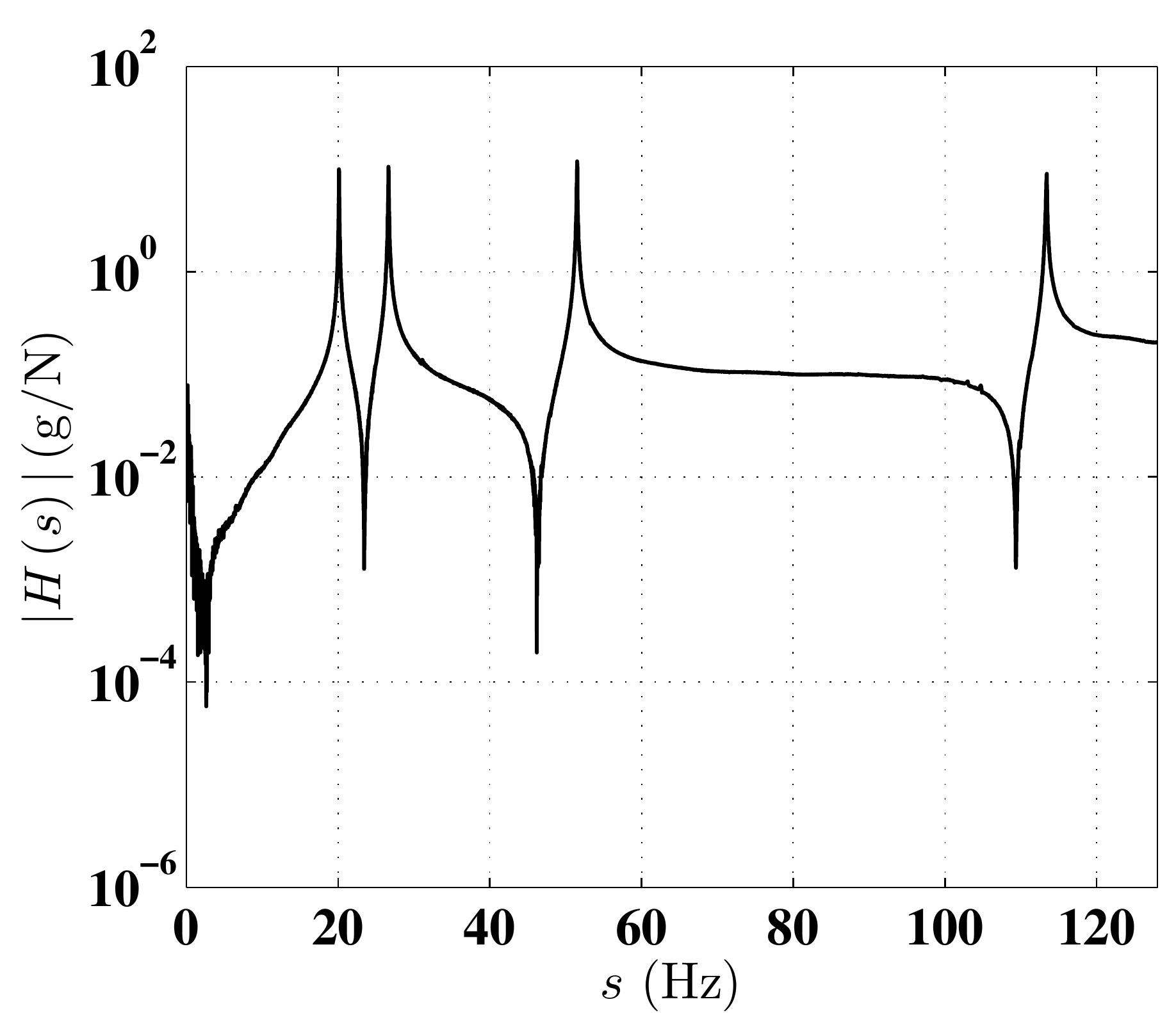

Figure 17c 
Figure $17 d$

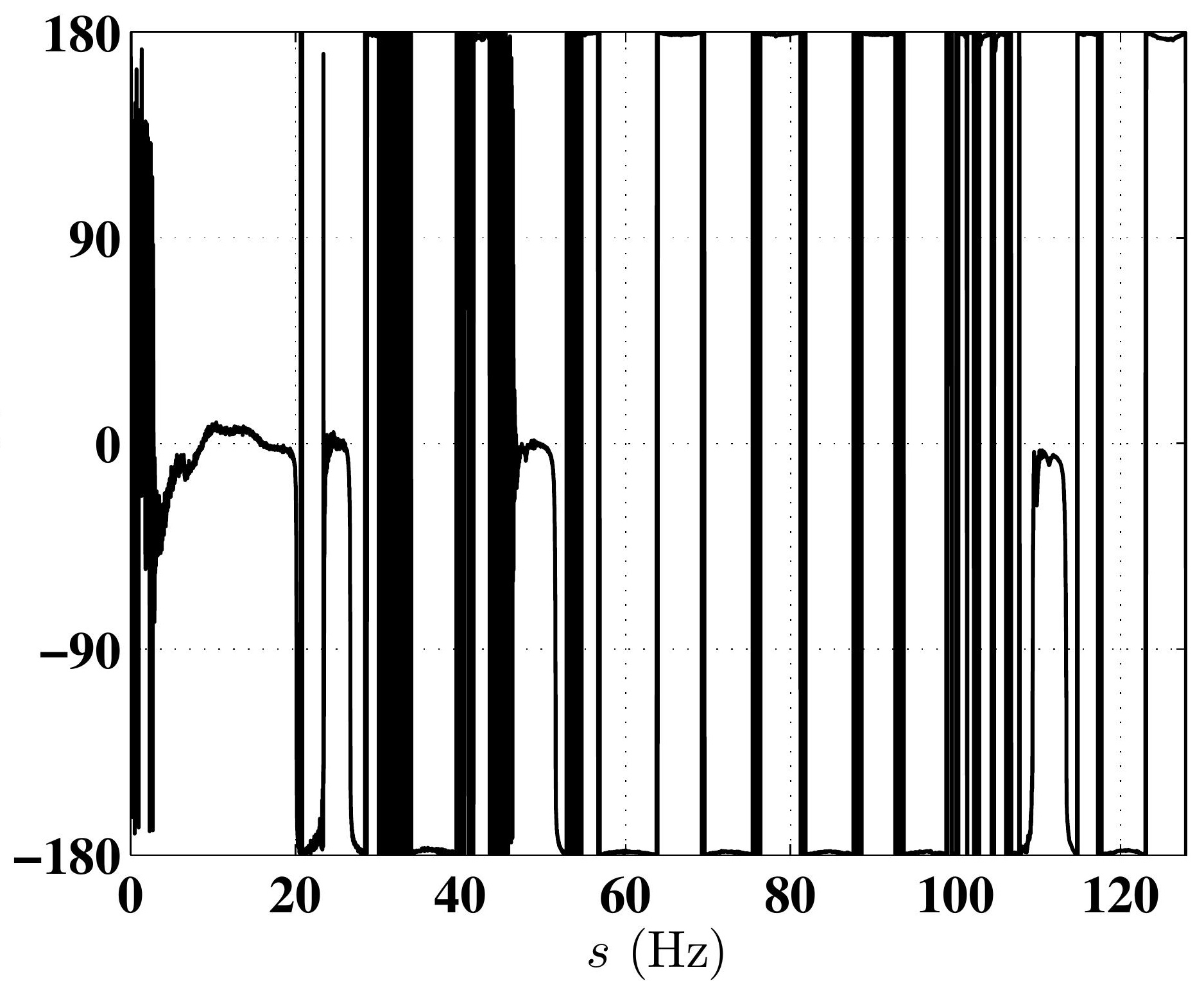


Figure 17e

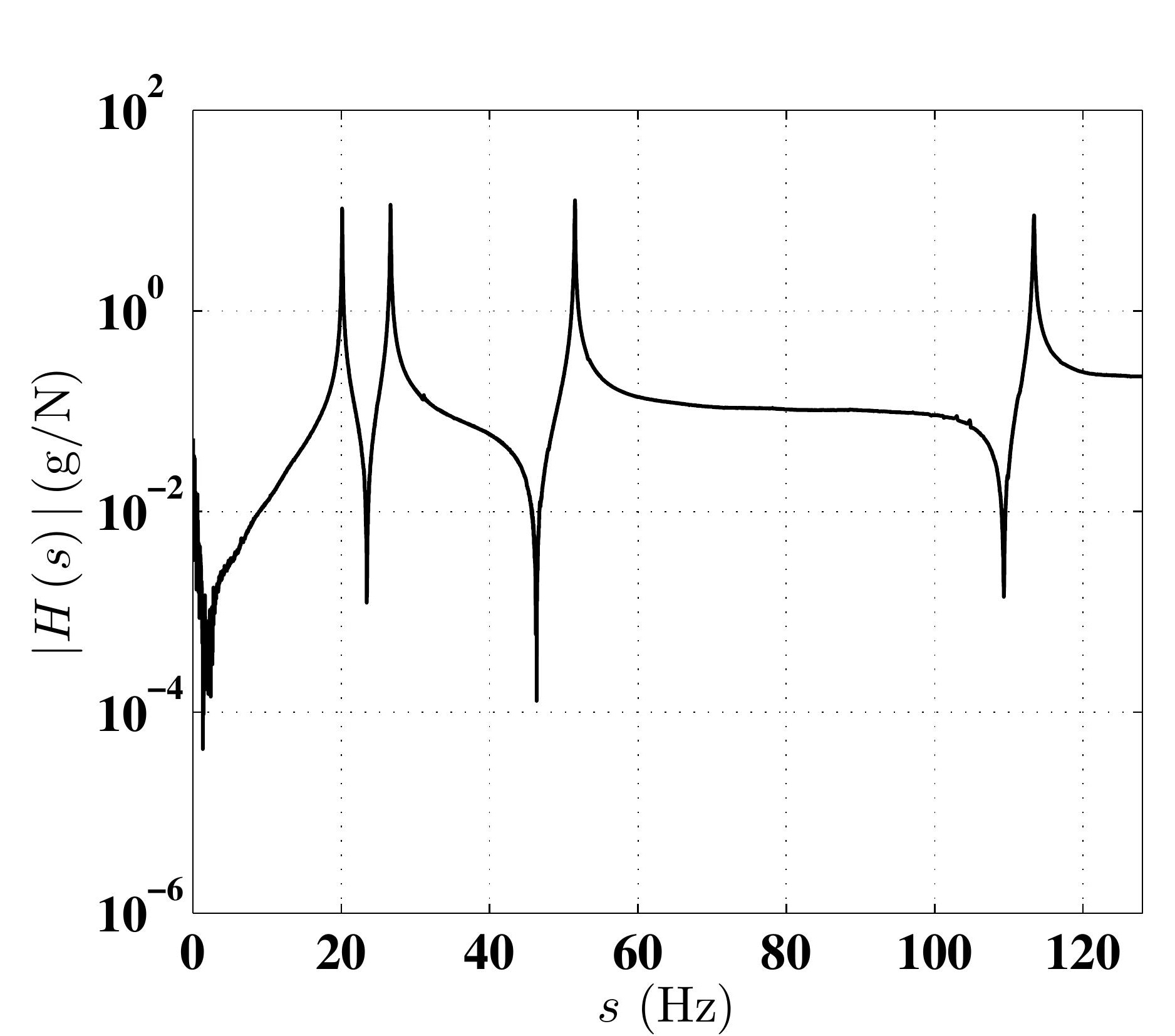

\section{.}

(
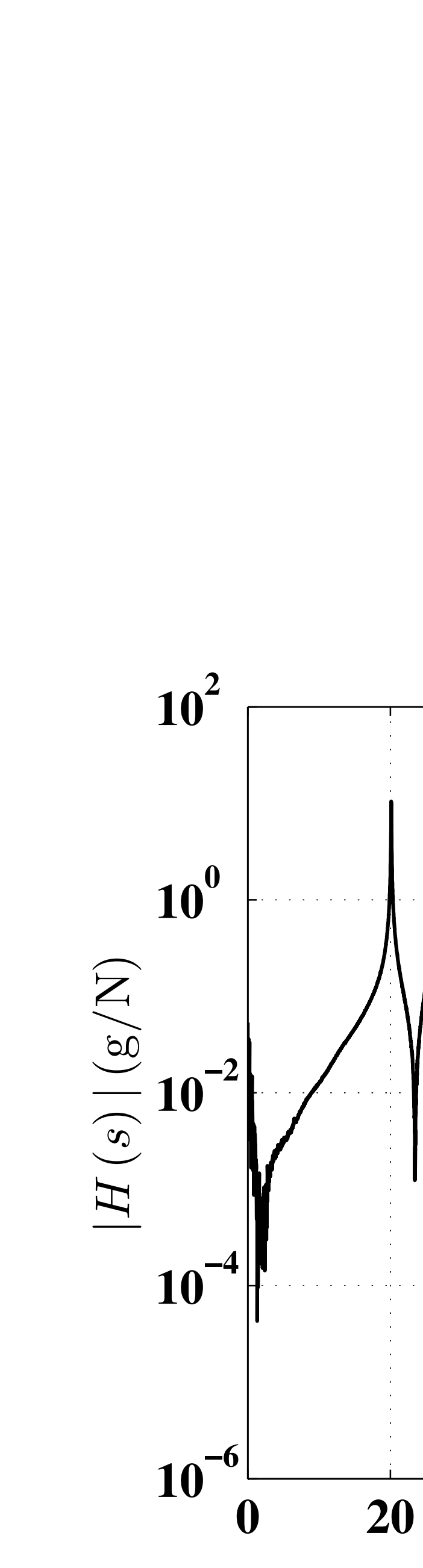
Figure $17 f$

180
90

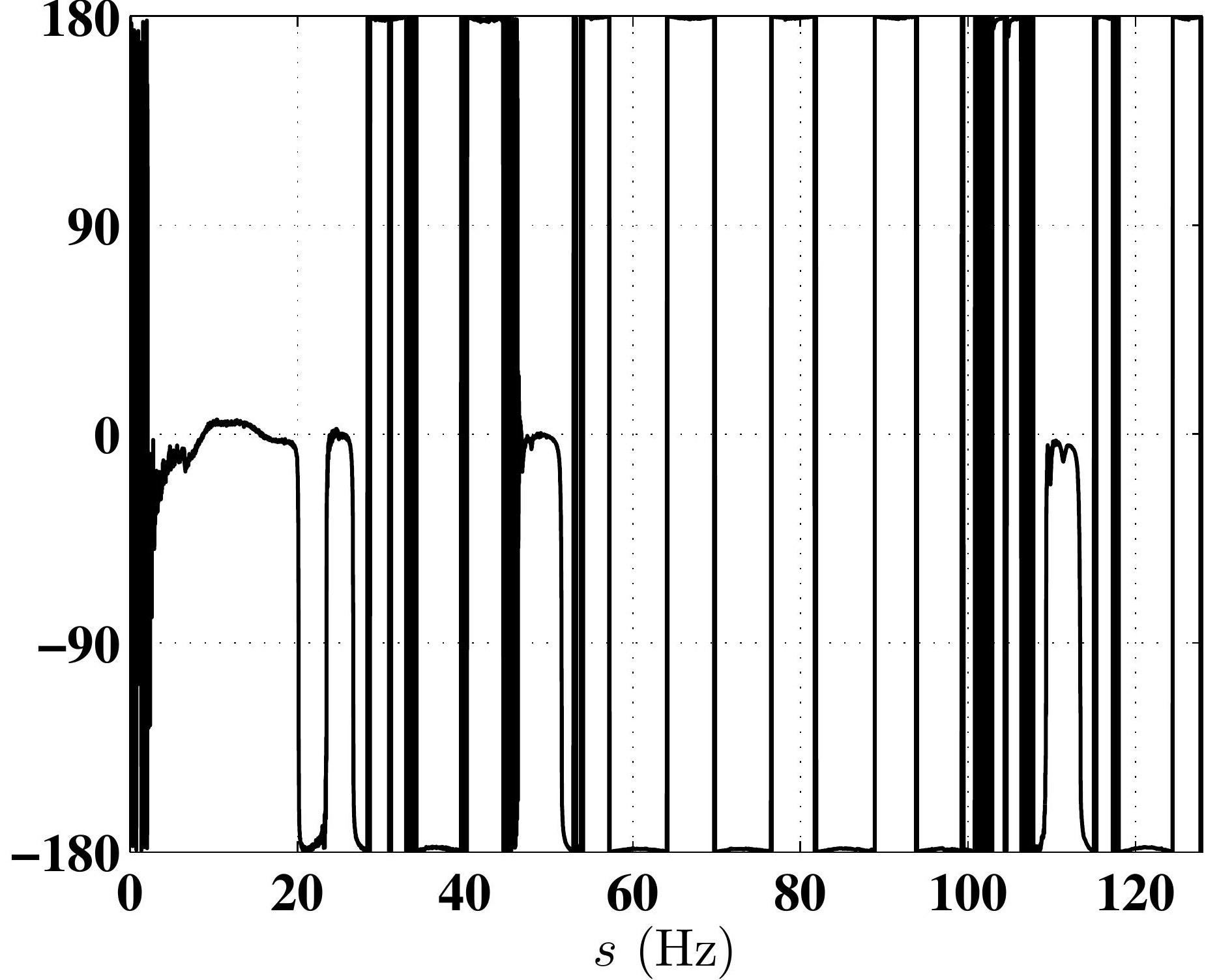

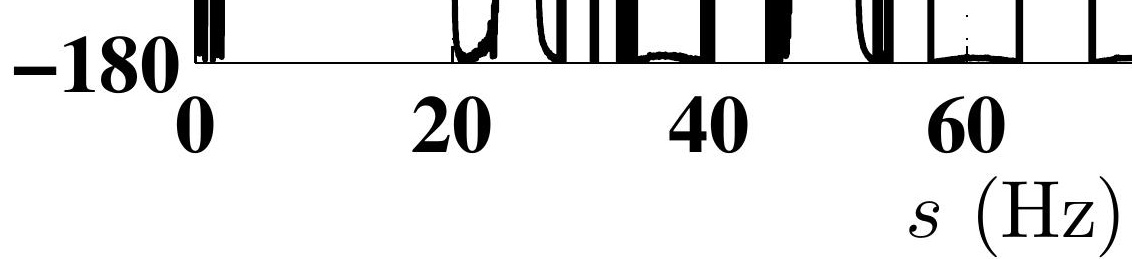




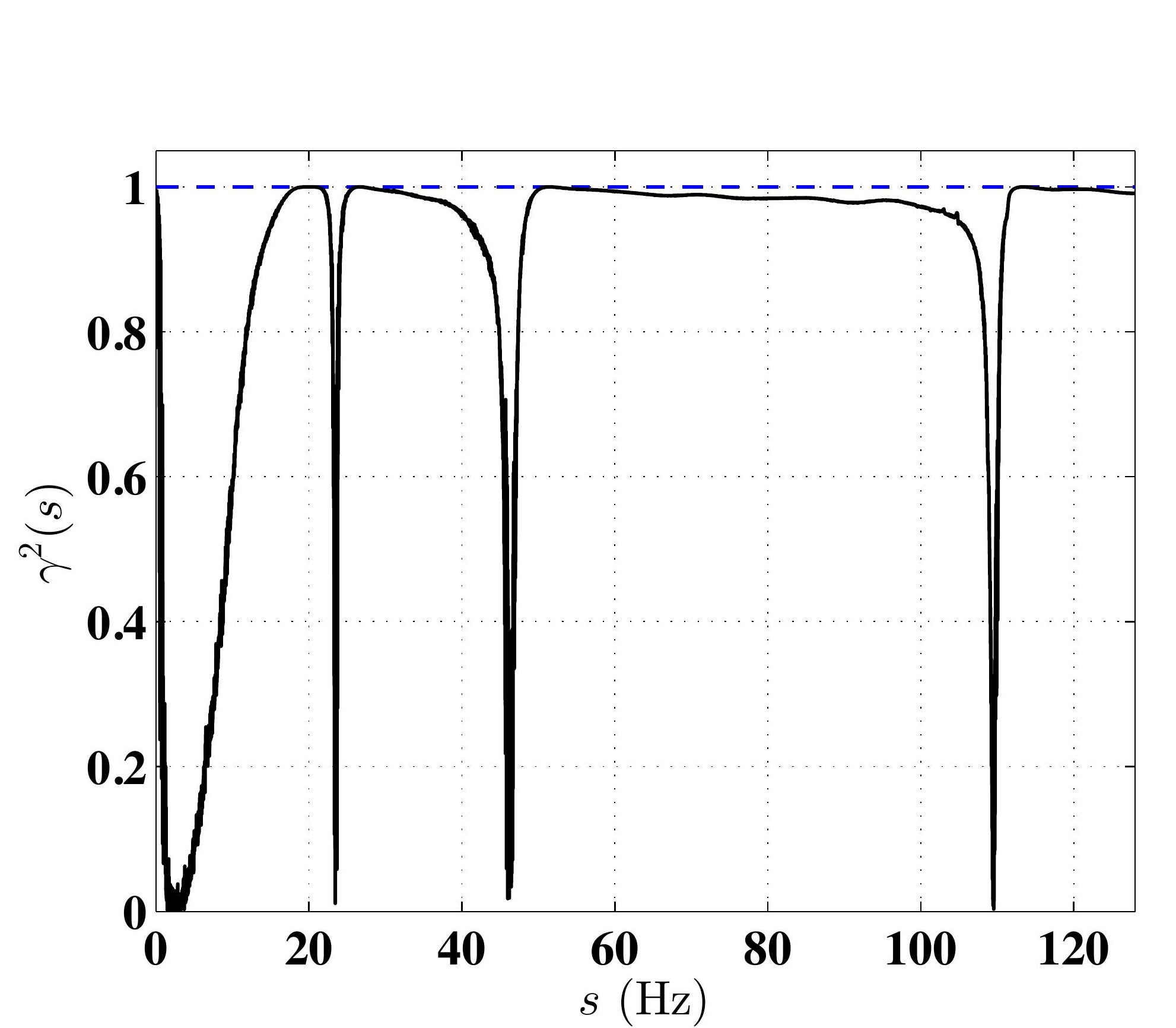

Figure 18a

\section{.}




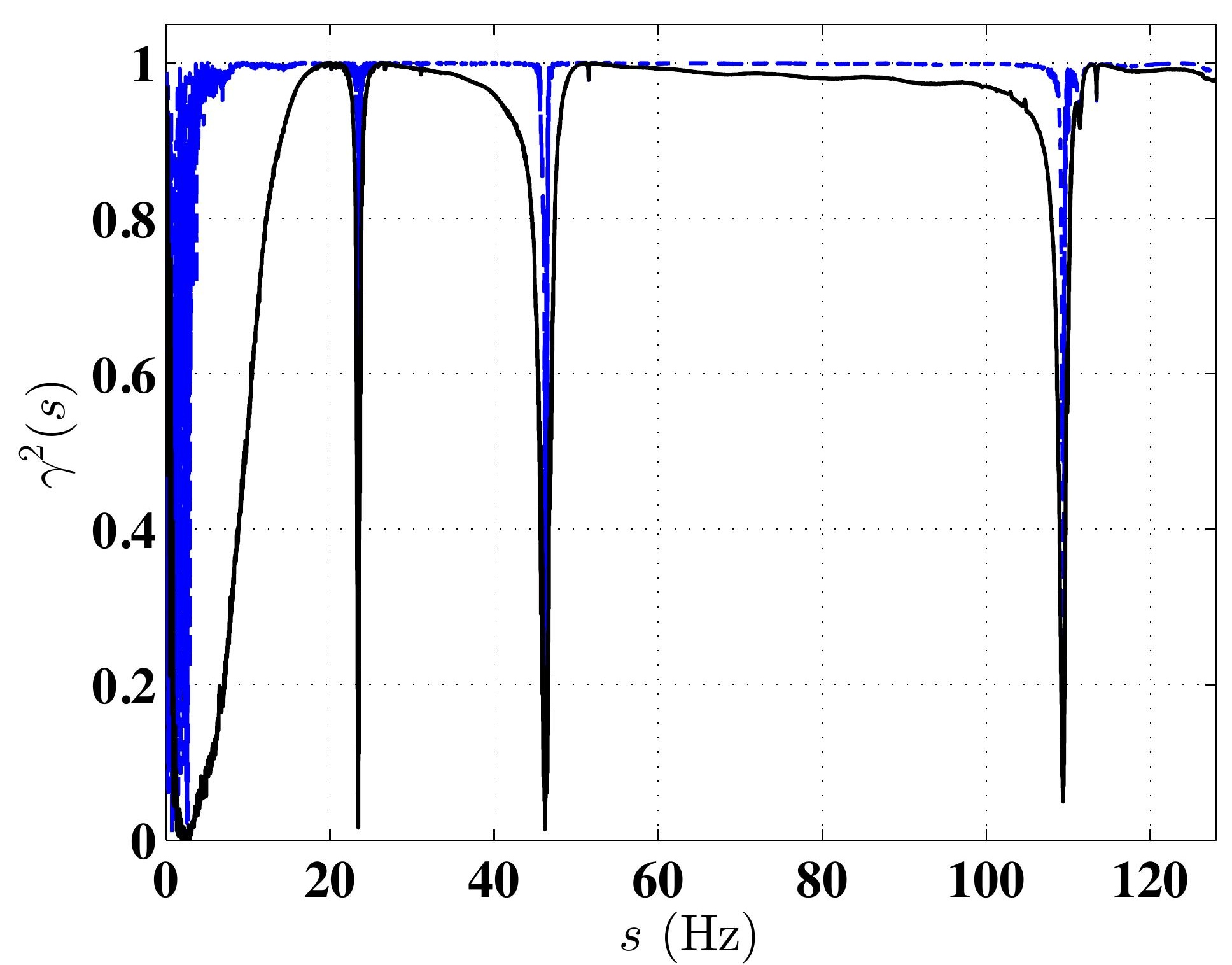

Figure $18 b$ 


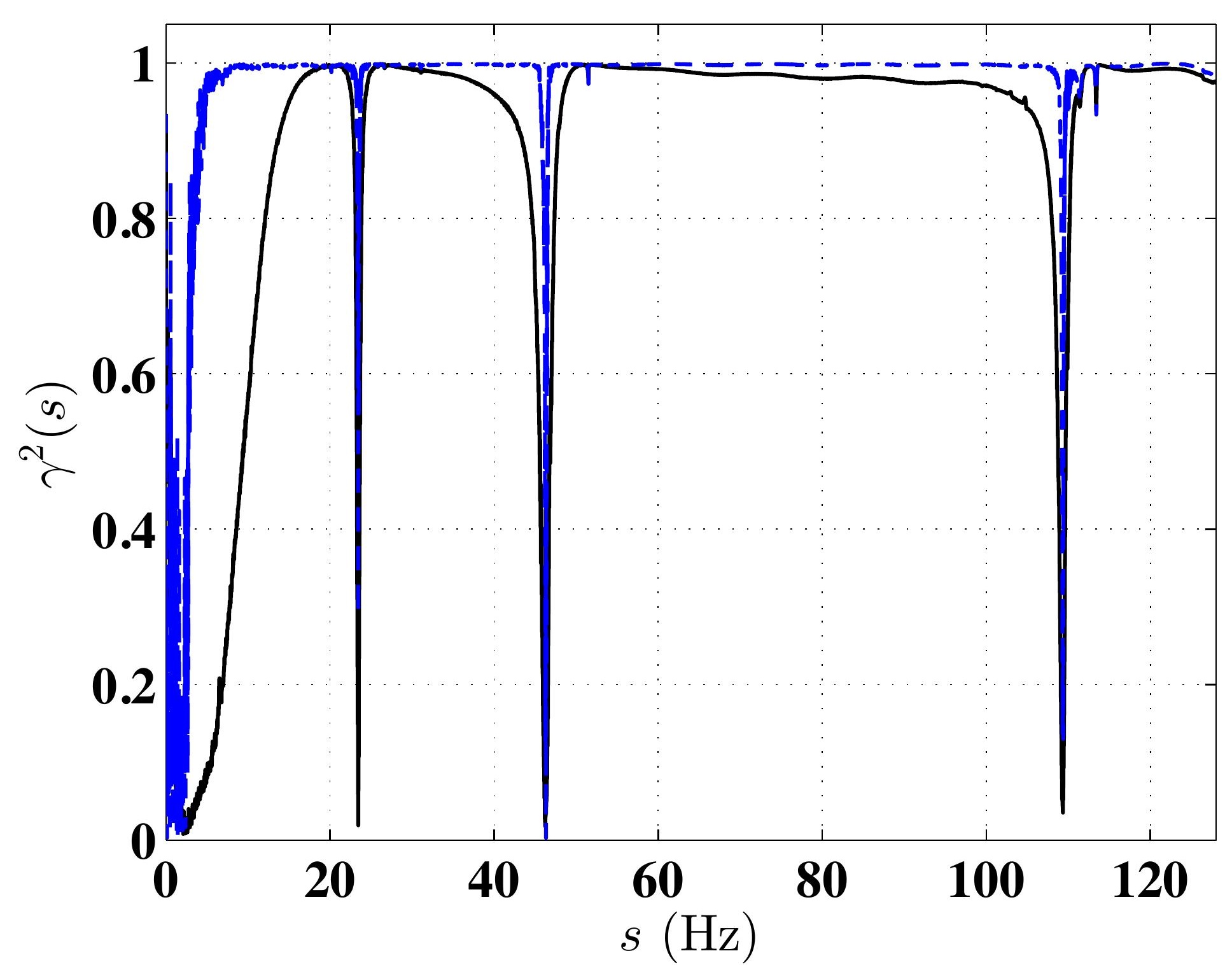

Figure $18 c$ 


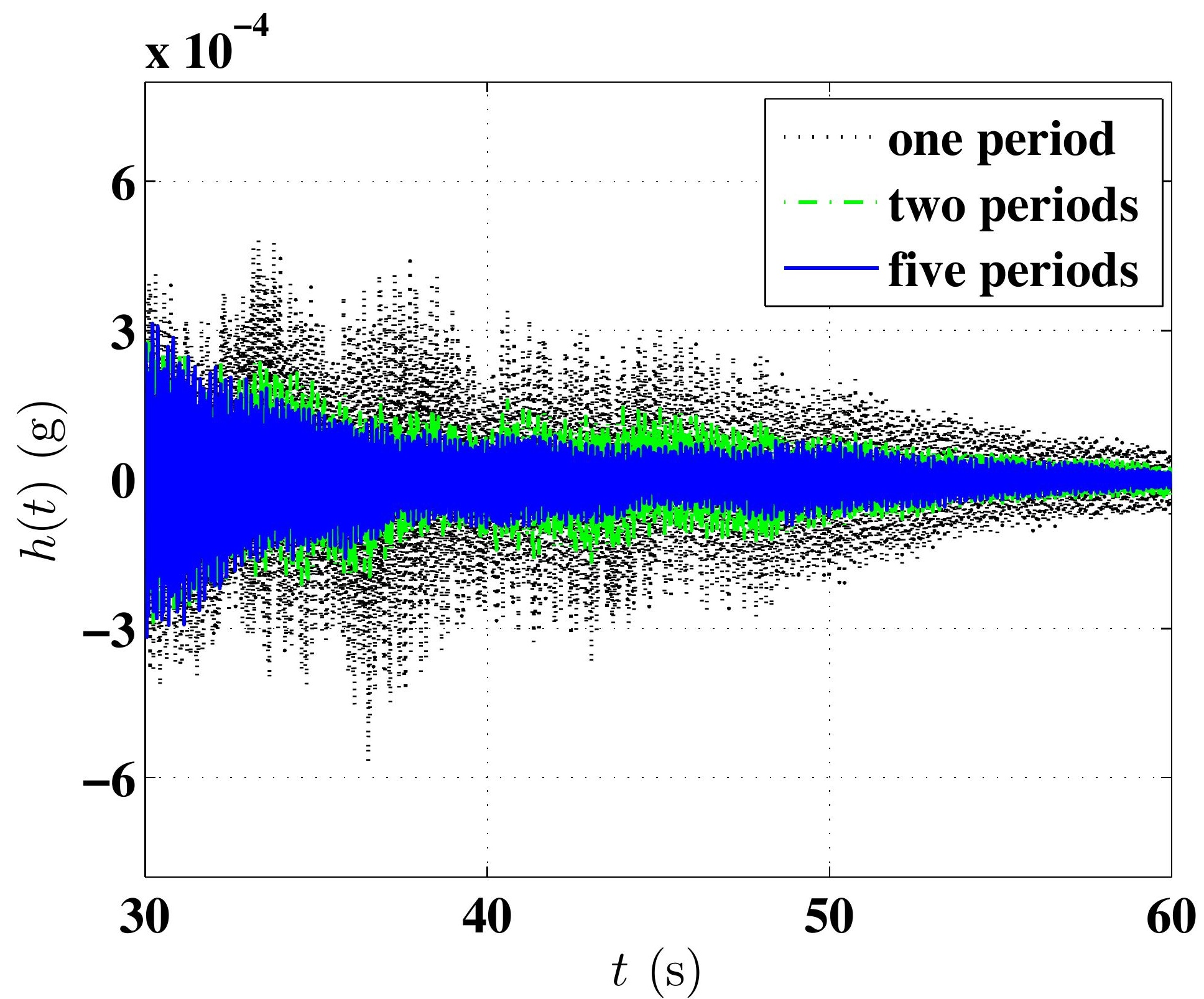




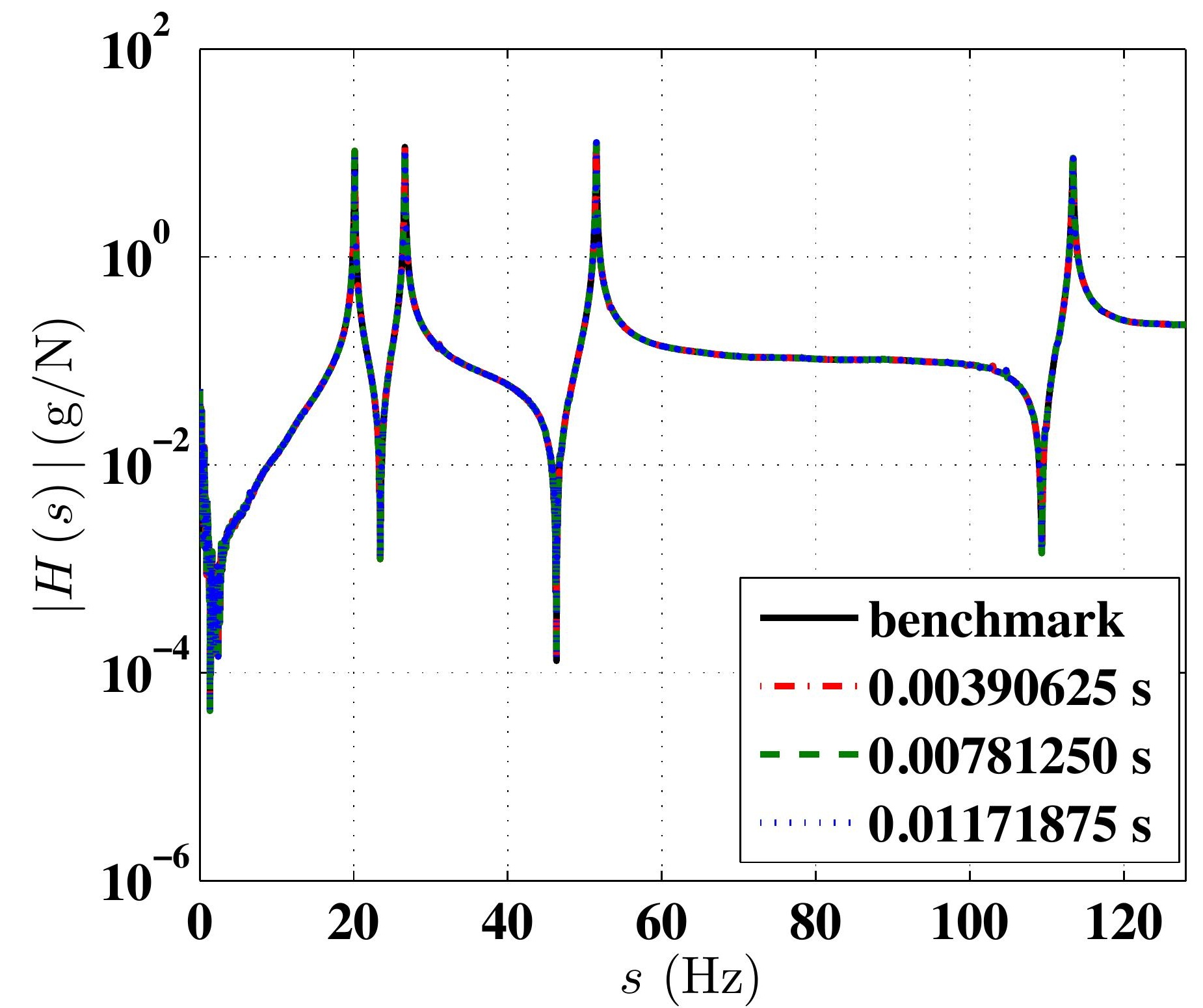

Figure 20a

$s(\mathrm{~Hz})$ 


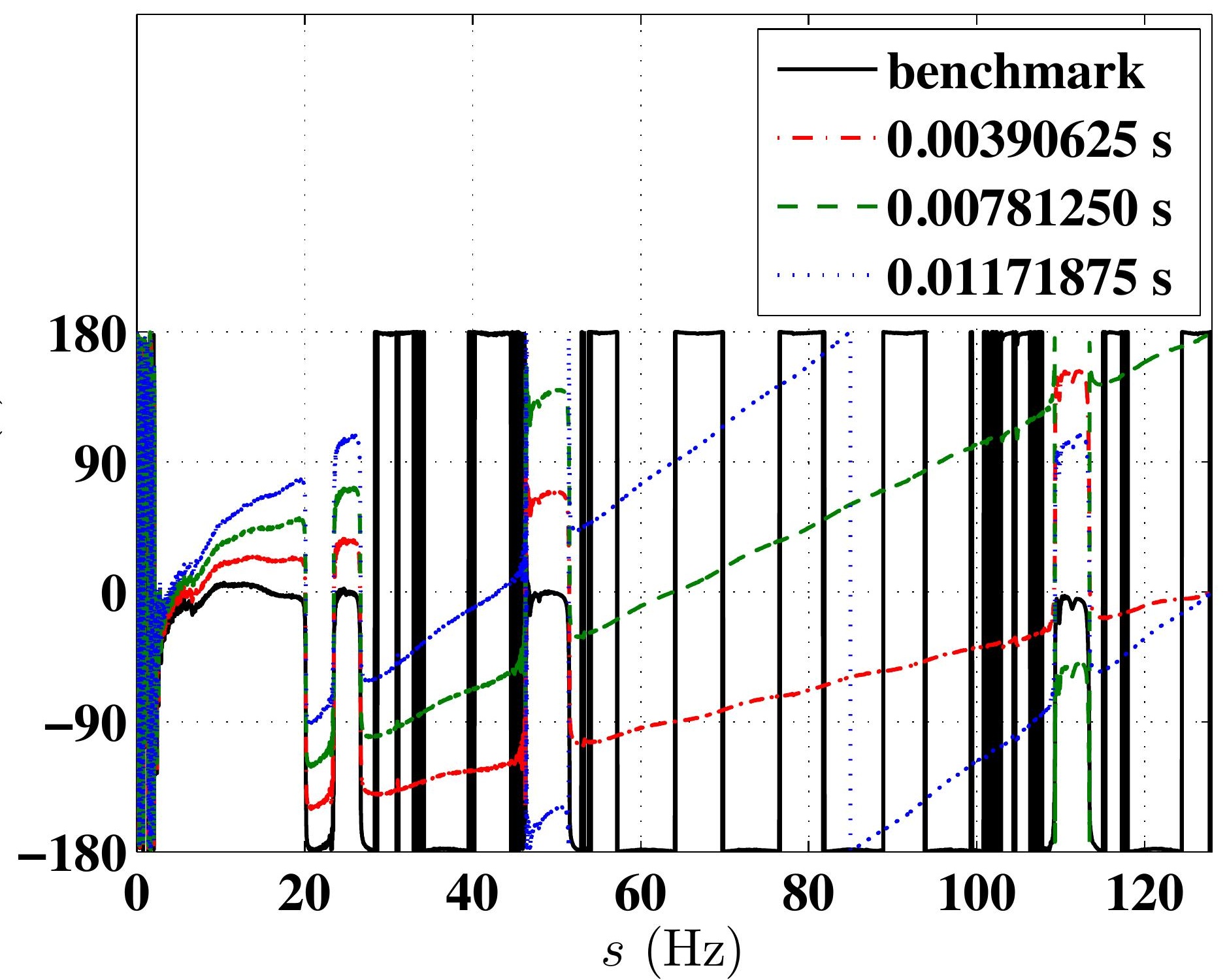




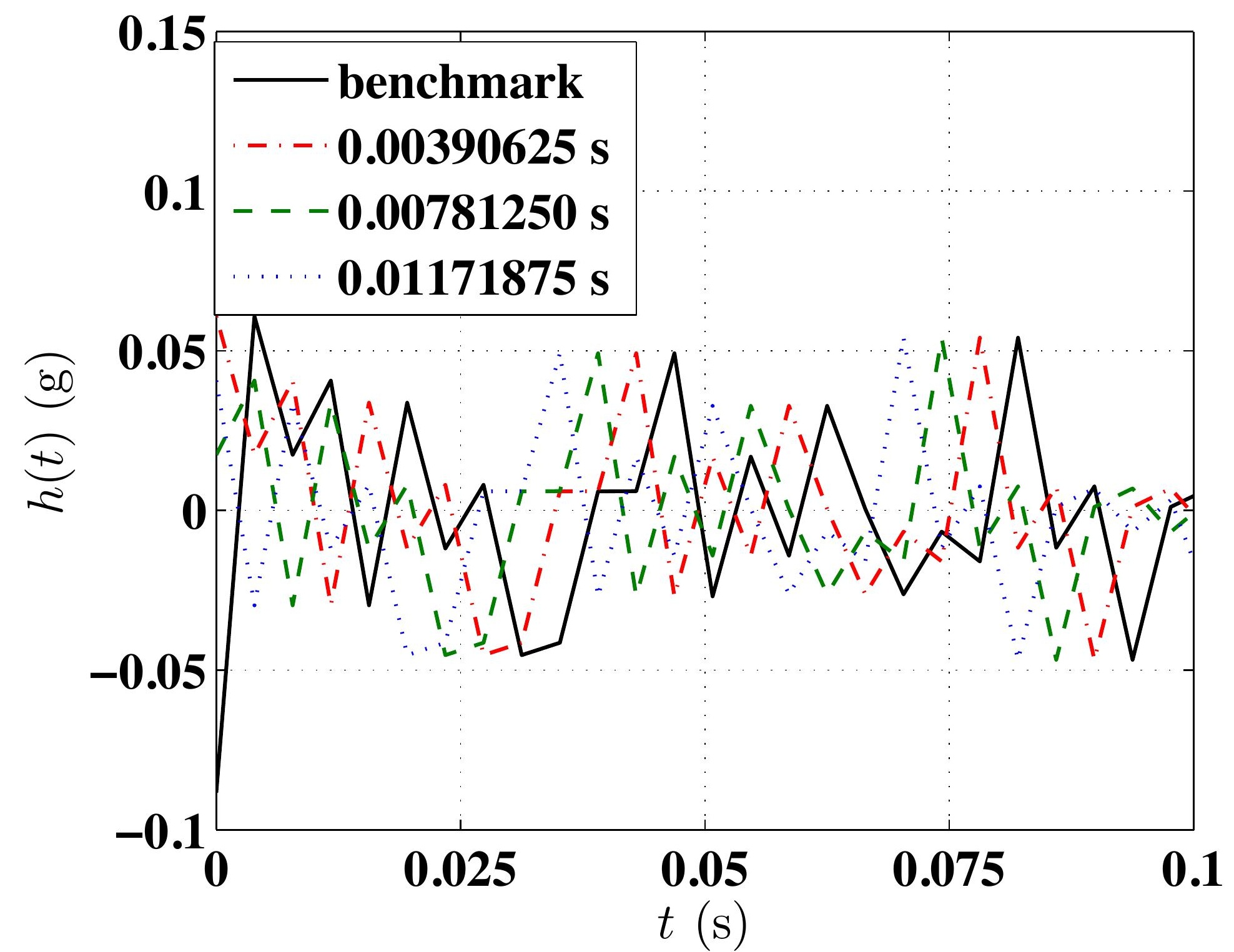


Figure 21b

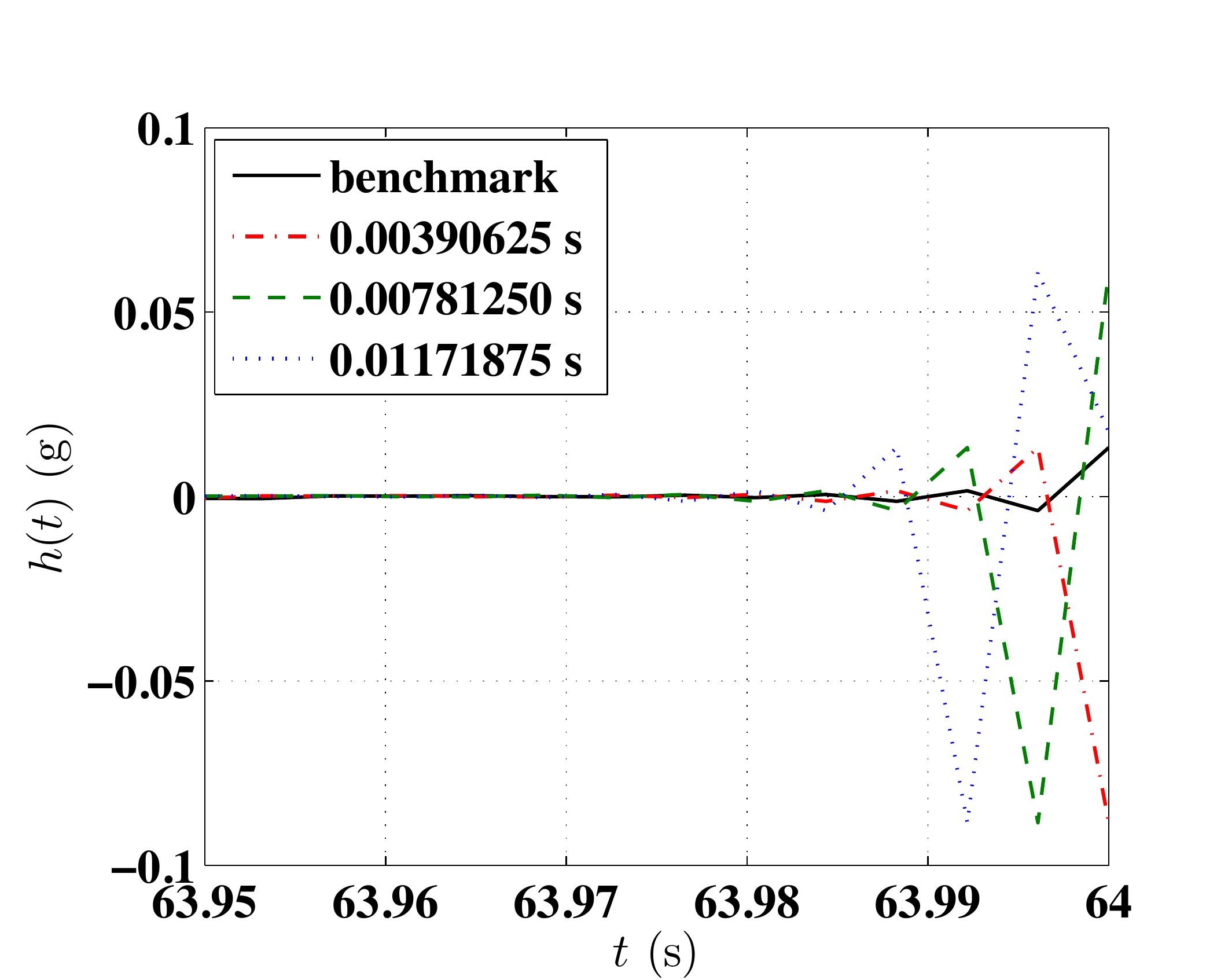


Figure 22a

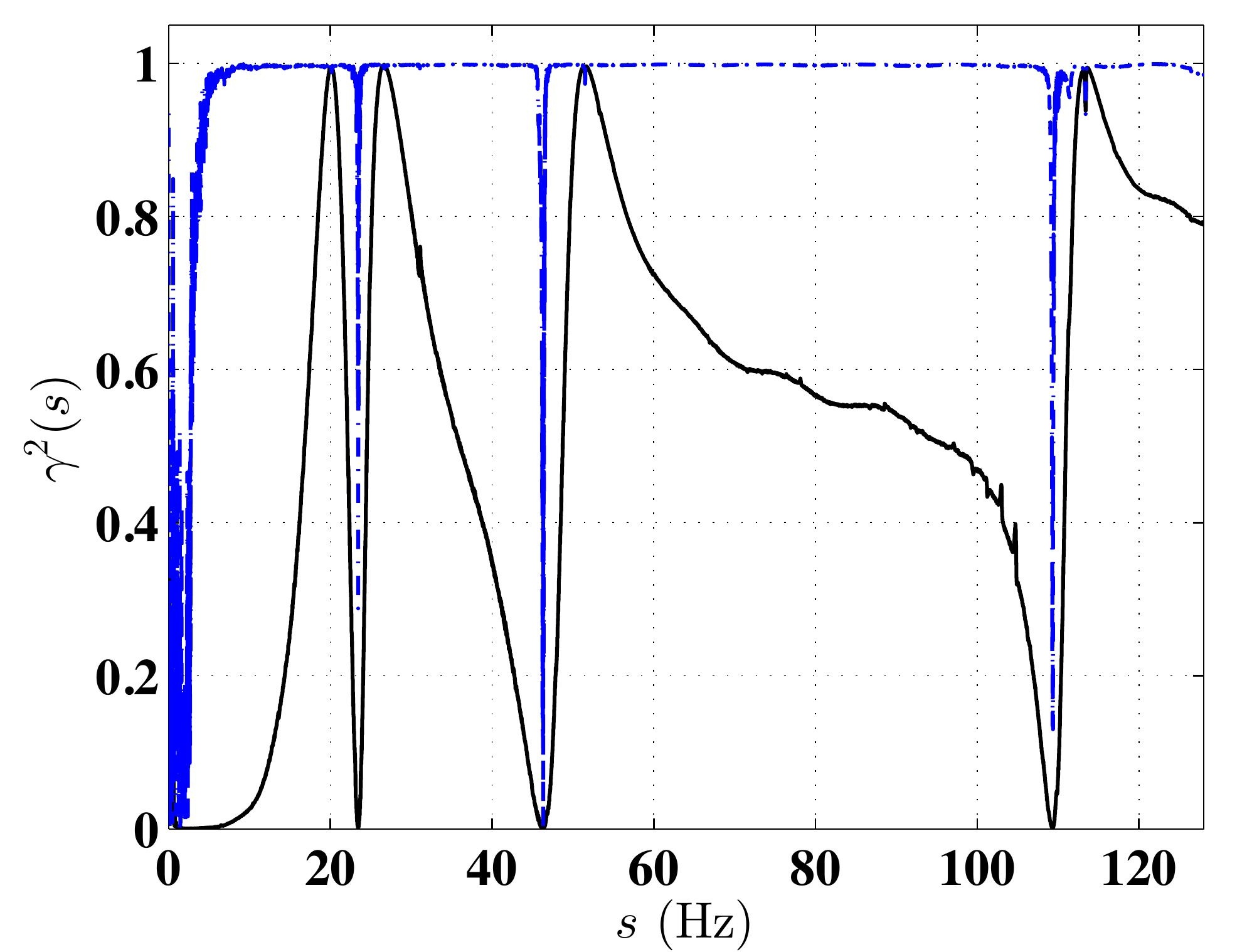




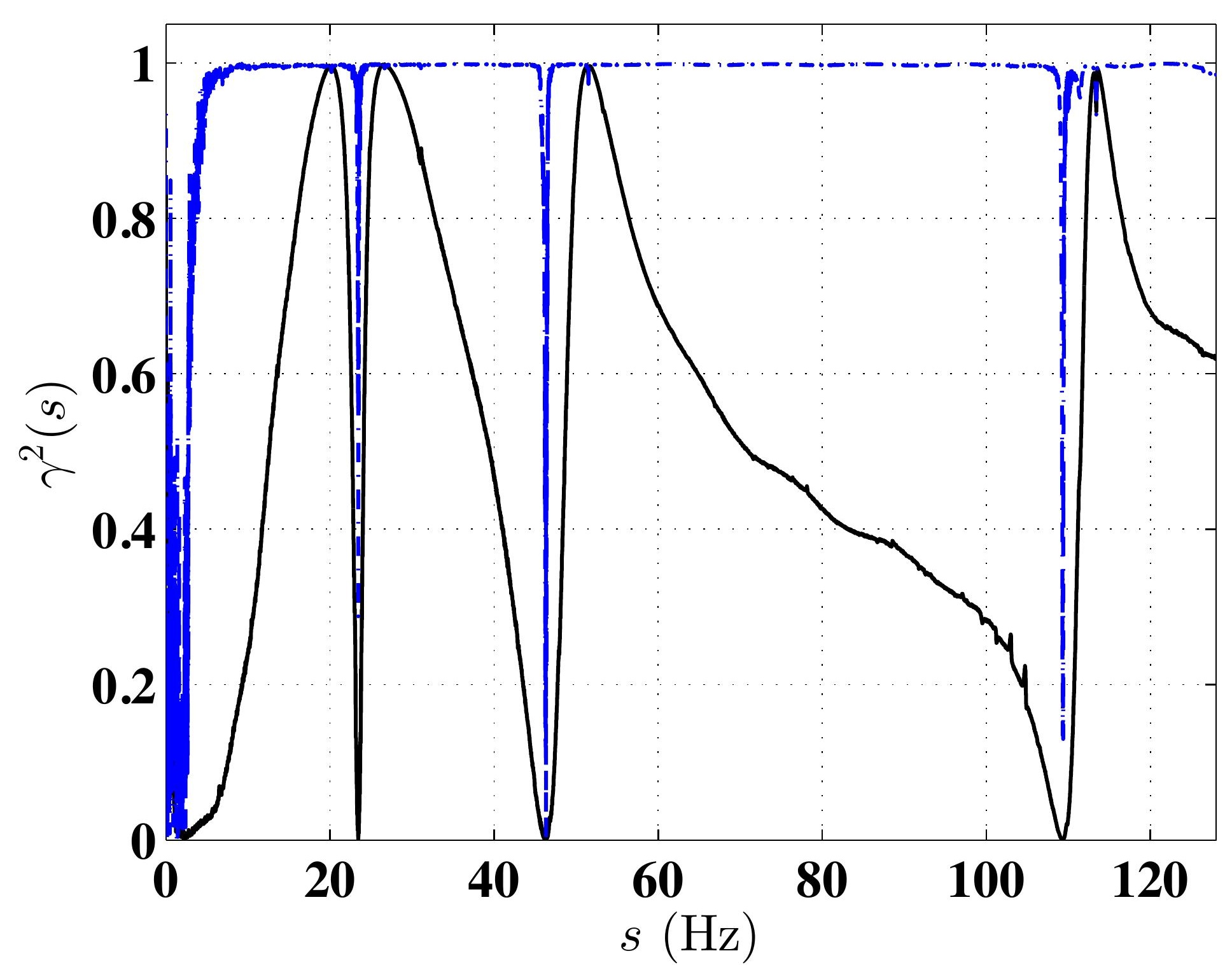

Figure $22 b$ 


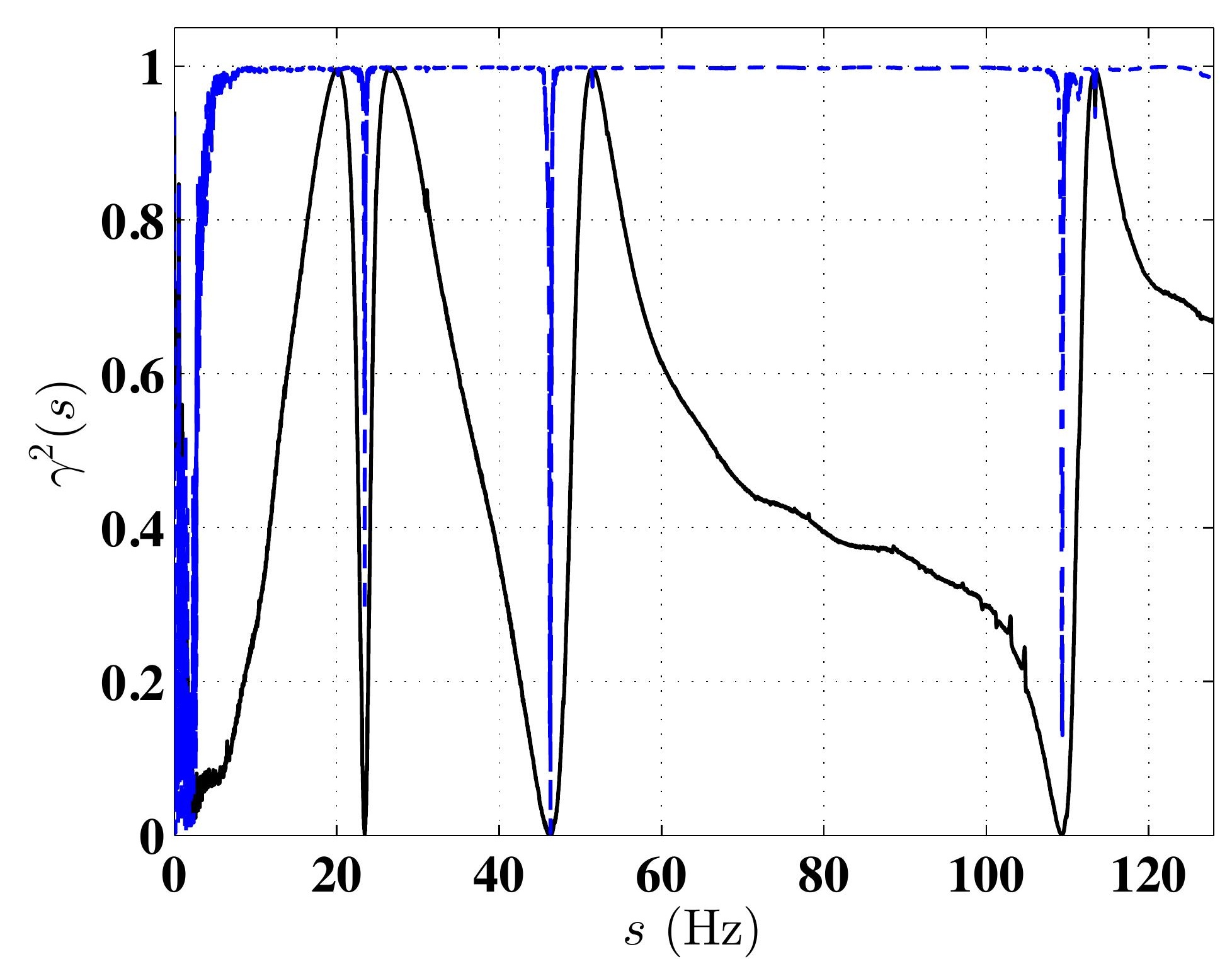

Figure 22c 
Highlights
Click here to download Supplementary Material: highlights_mssp_rev.docx

.

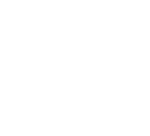

$\sqrt{2}$

(1)

(1)

(1)

(1)

(1)

(1)

.

.

.

.

.

.

.

.

.

.

.

.

.

.

.

.

.

.

.

.

.

.

.

.

.

.

.

. 
LaTeX Source File---Bib File
Click here to download LaTeX Source Files: aipsamp.bib

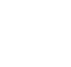

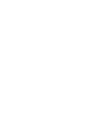

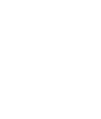

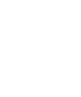

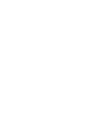

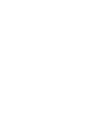

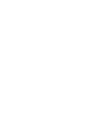

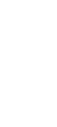

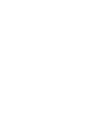

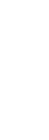

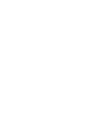
. TTeX Source Files: aipsamp.bib

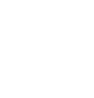

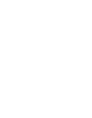

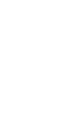
. . t - n

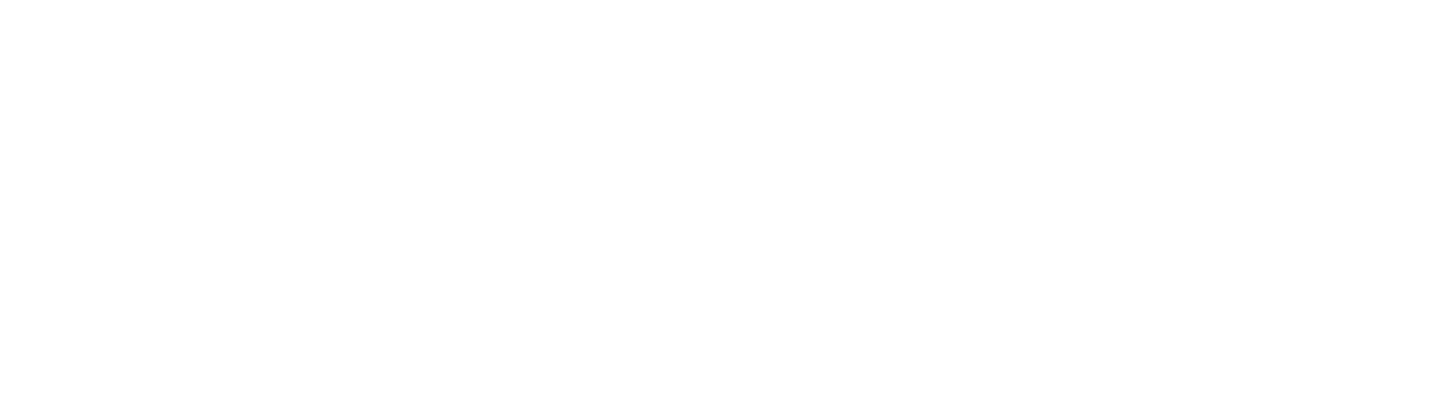

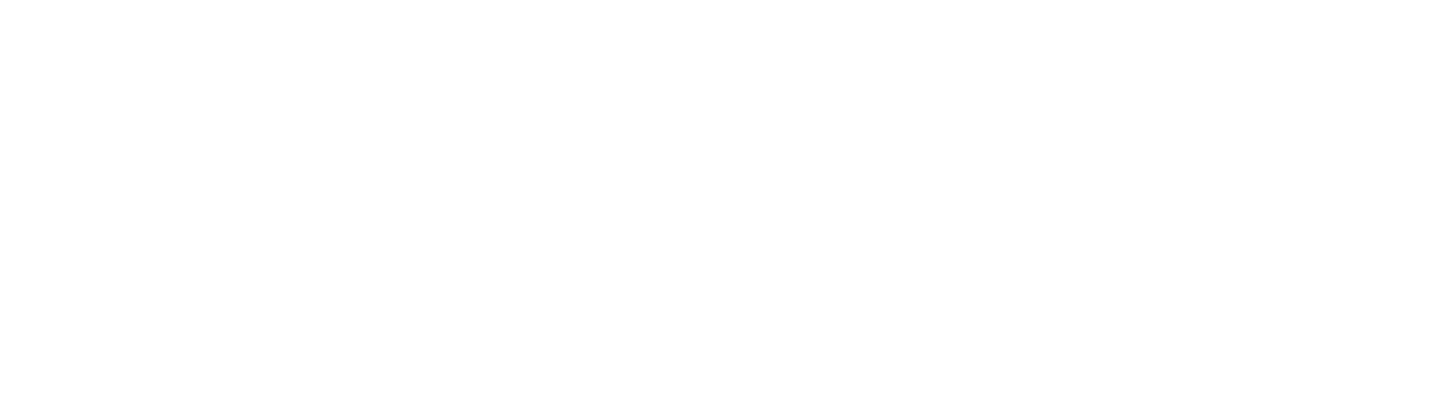

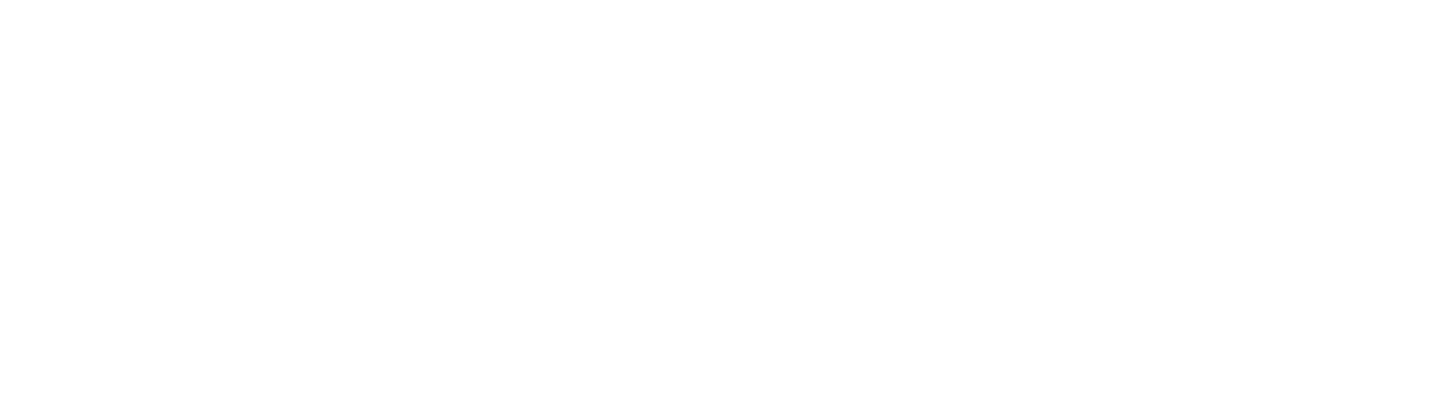

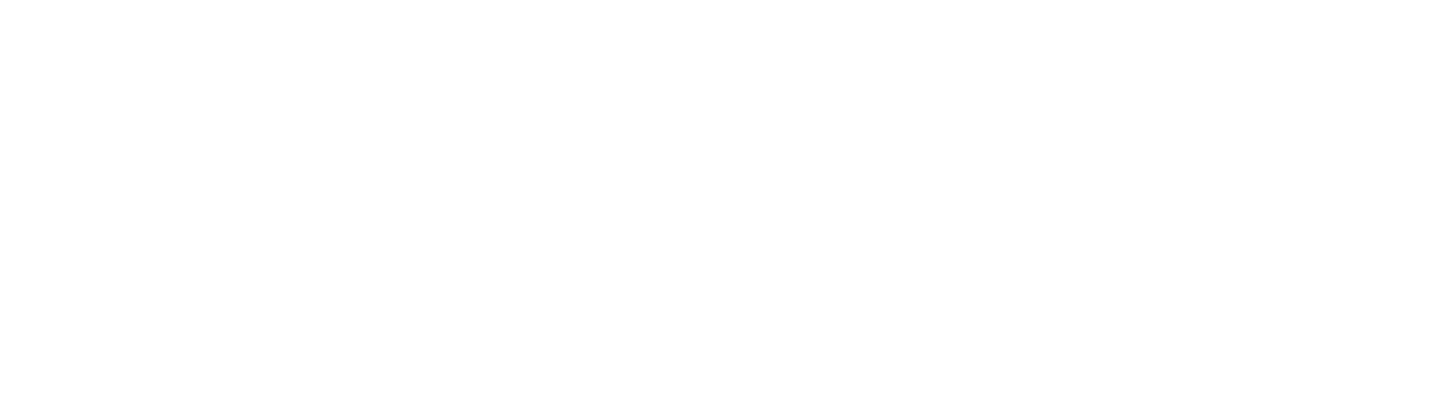

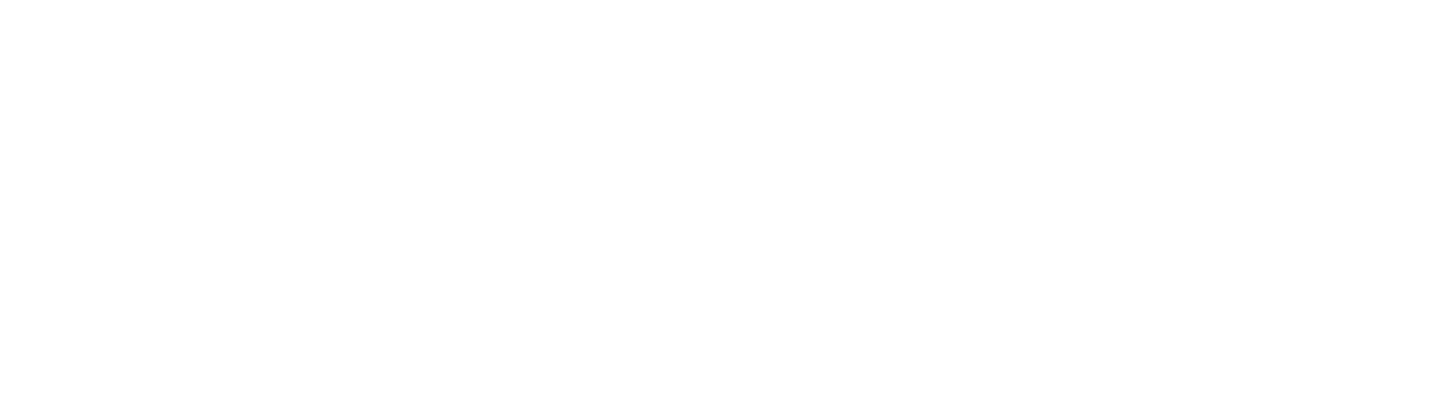

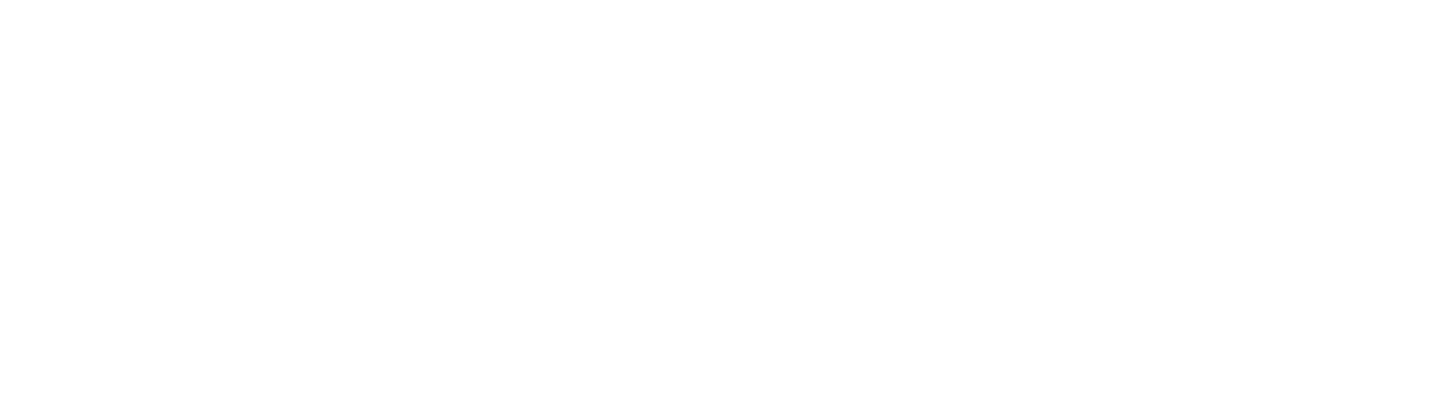

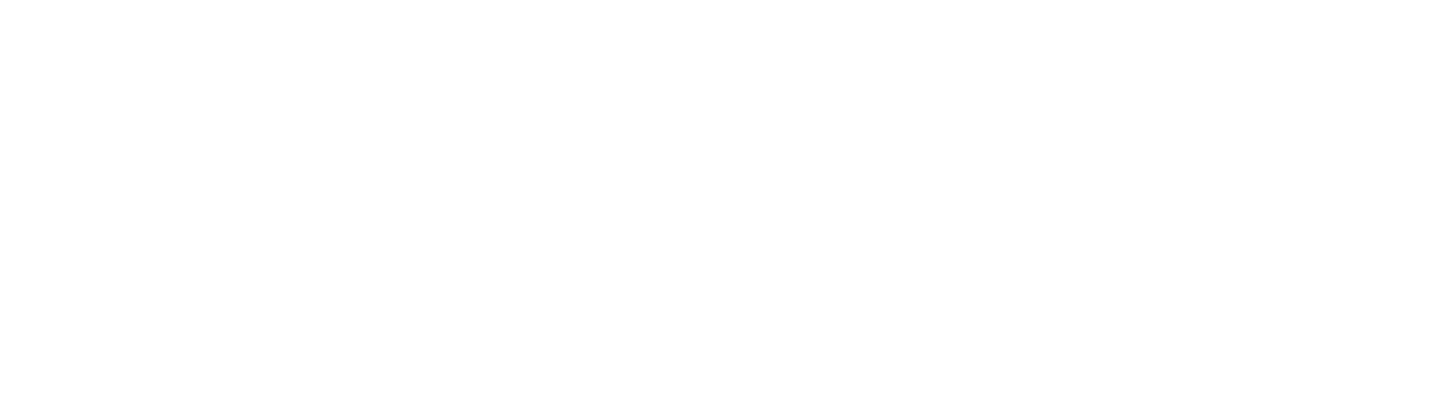

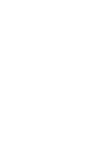
. 
